Bulletin of the Natural History Museum, 2019, 12: 37-84.

Received 22 Jun 2019; Accepted 22 Nov 2019.

doi:10.5937/bnhmb1912037J

UDC: 069.51:58/59(497.11)

Original scientific paper

\title{
DISTRIBUTION OF SELECTED VASCULAR PLANTS, FUNGI, AMPHIBIANS AND REPTILES IN SERBIA - DATA FROM BIOLOGICAL COLLECTIONS OF THE NATURAL HISTORY MUSEUM IN BELGRADE IMPLEMENTED IN CURRENT NATIONAL CONSERVATION PROJECTS
}

\author{
MiroslaV JoVANOVIĆ, UROŠ BUZUROVIĆ, BORIS IVANČEVIĆ, \\ ANA PAUNOVIĆ, MARJAN NIKETIĆ* \\ Natural History Museum, Njegoševa 51, 11000 Belgrade, Serbia, \\ e-mail: mniketic@nhmbeo.rs
}

A total of 990 records of selected vascular plants, fungi, amphibians and reptiles from biological collections of the Natural History Museum in Belgrade was reporter for Serbia. They include 34 representatives of Polypodiopsida, 32 of Pinopsida, 560 of Magnoliopsida, 127 of Pezizomycetes, 28 of Agaricomycetes, 16 of Amphibia and 13 of Reptilia. The databases contains 86 species of vascular plants, 24 species of fungi, 13 species of amphibians and 8 species of reptiles which are protected and strictly protected in Serbia.

Key words: vascular flora, fungi, amphibians, reptiles, protected species, Serbia

\section{INTRODUCTION}

This catalog is the result of the work of authors from Natural History Museum in Belgrade who participated in the development of current 
conservation projects funded by the Institute of Nature Conservation of Serbia: 1) "Obtaining data and other services in order to continue establishing an ecological network in the Republic of Serbia" JNOP 01/2018; 2) "Obtaining data and other services for the purpose of establishing the ecological network of the European Union Natura 2000 as part of the ecological network of the Republic of Serbia" JNOP 02/2018; 3) "Data acquisition and other services for the purpose of continuing the development of Red Data Lists of individual groups of flora, fauna and fungi in the Republic of Serbia" JNOP 03/2018. The project manager was prof. dr. Dmitar Lakušić from Faculty of Biology, University of Belgrade. Among other tasks, the authors have provided data on the distribution of selected vascular plants, fungi, amphibians and reptiles in Serbia that have been used for mapping endangered habitats and taxa, which are protected nationally or internationally or are listed in particular lists of endangered, rare or chorologically interesting taxa. The main objectives of these projects include the conducting of inventory of flora, fungia and fauna of Serbia, the implementation of existing data on significant species and habitats in key activities for the protection of biodiversity in Serbia, as well as in the documents necessary for the successful implementation and closure of Chapter 27 - Environment within the framework of Serbia's accession to the European Union.

The Natural History Museum in Belgrade was founded in 1895, and is now one of the oldest and largest natural history institutions in Southeast Europe, both in richness and diversity of the collection. Its biological collections comprised of 117 natural history collections with approximately 750 thousand specimens. Most of them are of regional importance and originate from Serbia and the Balkan Peninsula, which are of particular biogeographical and biohistorical importance. There are also specimens that have been collected worldwide. Collected and preserved objects from nature accomplish their purpose through museological and scientific processing and evaluation. The General Herbarium of the Balkan Peninsula (BEO) comprises more than two hundred thousand samples of vascular flora and ca. 25 thousand samples of fungi. In the Study collection of amphibians there are ca. 900 samples and in the Study collection of reptiles about 550 samples are registered.

\section{MATERIAL AND METHODS}

Input mode for entered database records was defined by the Standards of the "Center for Biodiversity Information" and internal Protocol on the collection, processing, organization and management of data. 
Revision and identification of the herbarium specimens was conducted by using Flora Europaea (Tutin et al. 1964, 1968-1980, 1993), regional flora (Josifović 1970-1977, Sarić 1992, Stevanović 2012) and other sources. Current nomenclature of plant names was proposed in accordance with the Euro+Med Plant Base (2006+), Plants of the World Online (POWO 2019) and Checklist of Flora Serbia (Niketić \& Tomović 2018).

Specimens from mycological collections were identified using descriptions and illustrations in Breitenbach \& Kränzlin (1984), Ceruti et al. (2003), Horak (2005), Montecchi \& Sarasini (2000), Moreno-Arroyo et al. (2005), Pegler et al. (1993), Zambonelli et al. (2016), etc. Fungal names were listed according to the Index Fungorum database (IF 2019).

Identification of amphibians and reptiles was done by the key given in Arnold \& Ovenden (1992) and nomenclature with current valid names was presented in accordance with ITIS (2019).

Acronyms for biological collections at the Natural History Museum in Belgrade: BEO - General herbarium of the Balkan Peninsula (listed on Index Herbariorum), vascular plants; BEO (Fungi) - Mycological collection of General herbarium; SZV - Study collection of amphibians; SZG Study collection of reptiles.

\section{RESULTS}

Original transcription from labels and records in databases is presented. Acronyms for Serbian regions: Bč - Bačka; Bt - Banat; C - Central Serbia; I - Eastern Serbia; J - Southern Serbia; JI - Southeastern Serbia; JZ Southwestern Serbia; K Kosovo; M - Metohija; Po - Great Pomoravlje; S Šumadija; SI - Northeastern Serbia; Sr - Srem; SZ - Northwestern Serbia; Z - Western Serbia.

\section{VASCULAR PLANTS}

\section{Polypodiopsida}

\section{ATHYRIACEAE}

\section{Athyrium filix-femina (L.) Roth}

C Kopaonik: Treska, UTM DP67 (leg. Rudski, I., 14.07.1938., det. Rudski, I., BEO 499); Stolovi: Čiker, Bukovik, UTM DP62 (leg. Jurišić, J. 01.06.1925., det. Jurišić, J., BEO 487); Trstenik: Medveđa, UTM EP01 (leg. Rudski, I. 08.1938., det. Rudski, I., BEO 483); Veliki Jastrebac, UTM EP40, bukovo-jelova šuma (leg. Diklić, N. 21.07.1959., det. Diklić, N., BEO 35496); Skudaljinski potok, UTM EP40, bukova šuma (leg. Diklić, N. 15.05.1959., det. Diklić, N., BEO 35497); zapadna padina, UTM EP40, Exp. W, bukova šuma (leg. Diklić, N. 20.07.1959., det. Diklić, N., BEO 35495); JI 
Ostrozub: klisura, UTM EN94, tresava (leg. Janković, M., 17.07.1950., det. Diklić, N., BEO 35509); Vlasina: Vlasinsko jezero, UTM FN03, šume breze i bukve (leg. Nikolić, V., Diklić, N. 17.06.1967., det. Diklić, N., BEO 35504); $\underline{M}$ Prokletije: Derviš kom, UTM DN20 (leg. Černjavski, P., Rudski, I., Lindtner, V., 16.07.1933., det. Černjavski, P., BEO 481); Mokra planina, Savina Reka, UTM DN54 (leg. Rudski, I. 17.07.1932., det. Rudski, I., BEO 500); SI Crni vrh, UTM EP78, bukova šuma (leg. Nikolić, V., 28.05.1955., det. Nikolić, V., BEO 35508); Deli Jovan: Crni vrh, UTM EP78, bukova šuma (leg. Nikolić, v. 17.06.1956., det. Nikolić, V., BEO 35507); Đerdap: Donji Milanovac, Paprinica reka, UTM EQ92, Exp. NW, mešovita šuma (leg. Nikolić, V., Diklić, N., Rakin, M. 12.09.1965., det. Diklić, N., BEO 35499); Varoški potok, UTM EQ82, Exp. NW, bukova šuma (leg. Diklić, N., Nikolić, V., Rakin, M. 11.09.1965., det. Diklić, N., BEO 35498); Papratnički potok, UTM FQ14, EQ73 (leg. Nikolić, V., Diklić, N. 25.09.1966., det. Diklić, N., BEO 35503); Porečka reka, Gradišnica potok, UTM EQ91 (leg. Nikolić, V., Diklić, N. 04.08.1966., det. Diklić, N., BEO 35500); Tekija, Kovilovski potok, UTM FQ14 (leg. Nikolić, V., Diklić, N. 26.06.1967., det. Nikolić, V., BEO 35505); (leg. Nikolić, V., Diklić, N. 26.09.1967., det. Nikolić, V., BEO 35506); Majdanpek: Majdanpečka domena, potok Spinje, UTM EQ71 (leg. Černjavski, P. 17.06.1946., det. Černjavski, P., BEO 497); Todorova Reka, UTM EQ61 (leg. Černjavski, P. 18.07.1947., det. Černjavski, P., BEO 496); SZ Jablanik: Vujnovača, UTM CP99 (leg. Jurišić, Ž., 21.06.1910., det. Jurišić, Ž., BEO 494); UTM CP99 (leg. Jurišić, Ž.

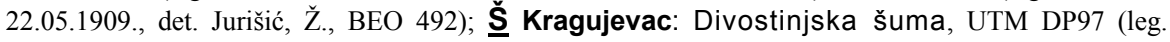
Rudski, .., 21.07.1936., det. Rudski, I., BEO 486).

\section{MARSILEACEAE}

\section{Marsilea quadrifolia $\mathrm{L}$.}

Bt Ečka: Novo jezero, UTM DR51, kamenjar (leg. Milovanović, D., 11.05.1949., det. Diklić, N., BEO 35676); Pančevo: Ivanovo - Mukulj rit, UTM DQ75, kanali (leg. Sigunov, A. 02.08.1975., det. Sigunov, A., BEO 35677).

\section{OPHIOGLOSSACEAE}

\section{Ophioglossum vulgatum L.}

I Svrljig: Plužine, UTM EP81 (leg. Lindtner, V., 18.06.1955., det. Diklić, N., BEO 136); Po Smederevska Palanka: Glibovac, Pridvorački lugovi, UTM DQ91 (leg. Lindtner, V., 07.04.1950., det. Lindtner, V., BEO 142); SI Beljanica: Busovata, UTM EP58 (leg. Lindtner, V., 24.05.1948., det. Lindtner, V., BEO 137); SZ Maljen: Divčibare, UTM DP28 (leg. Lindtner, V., 08.07.1948., det. Lindtner, V., BEO 140); Slavkovica: Rajac, UTM DP38 (leg. Lindtner, V. 20.05.1954., det. Diklić, N., BEO 135); Valjevo: Lukavac-Divci, UTM DQ20 (leg. Lindtner, V. 04.06.1949., det. Diklić, N., BEO

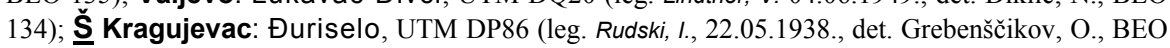
141).

\section{SALVINIACEAE (AZOLLACEAE)}

Salvinia natans (L.) All.

Bč Sombor: kamen kralja Petra, UTM CR56 (leg. Izmajlov, N., 08.1926., det. Izmajlov, N.,

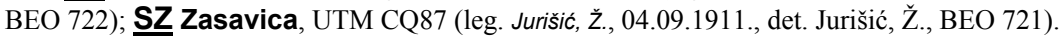




\title{
Pinopsida
}

\author{
PINACEAE
}

\section{Pinus heldreichii Christ}

JZ Kamena gora: Šajna kraj, južno od vrha planine, UTM CN89 (leg. Pavlović, Z., 20.04.1949., det. Pavlović, Z., BEO 989); Šajnov kraj, južno od vrha planine, UTM CN89 (leg. Pavlović, Z. 20.04.1949., det. Pavlović, Z., BEO 1050); K Šar-planina: Brezovica-Borovik, UTM EM07, serpentinit (leg. Nikolić, V., Diklić, N., Bogdanović, M., 29.07.1972., det. Nikolić, V., BEO 35770); M Koritnik: Ravnište, UTM DM66 (leg. Nikolić, V.,Diklić, N., 28.07.1968., det. Nikolić, V., BEO 35768); Prokletije: Koprivnik, UTM DN31 (leg. Černjavski, P. 08.1934., det. Černjavski, P., BEO 1029); Nemoris, UTM DN31, 2200 m, in glareosis (leg. Grebenščikov, O. 13.07.1939., det. Grebenščikov, O., BEO 1047); Mokra planina, UTM DN54 (leg. Rudski, I. 16.07.1932., det. Rudski, I., BEO 981); Berim, UTM DN64 (leg. Rudski, I. 17.07.1932., det. Rudski, I., BEO 1031); Džora, Seunovo, UTM DN43 (leg. Rudski, I. 28.06.1932., det. Rudski, I., BEO 1033); Veliki (Vrelski) potok, ispod visa, UTM DN54 (leg. Rudski, I. 02.07.1932., det. Rudski, I., BEO 1028); Vrelski potok, UTM DN54 (leg. Rudski, I. 02.07.1932., det. Rudski, I., BEO 988); Paklen, Kukavica, UTM DN32 (leg. Černjavski, P. 08.1934., det. Černjavski, P., BEO 1043); Z Murtenica: Ćuletina, UTM DP02, Exp. SE (leg. Pavlović, Z., 10.04.1948., det. Pavlović, Z., BEO 1012); ispod vrha na putu za Negbine, UTM DP02 (leg. Pavlović, Z. 22.07.1947., det. Pavlović, Z., BEO 1010); jugozapadne padine, UTM DP02, Exp. SW (leg. Pavlović, Z. 28.04.1949., det. Pavlović, Z., BEO 1017); na južnoj strani, UTM DP02, Exp. S (leg. Pavlović, Z. 10.04.1948., det. Pavlović, Z., BEO 1009); vrh, UTM DP02, Exp. E (leg. Pavlović, Z. 28.05.1944., det. Pavlović, Z., BEO 1013); Ćuletine, južne padine iznad sela Negbine, UTM DP02, Exp. S (leg. Popović, S. 28.04.1949., det. Pavlović, Z., BEO 1021); jugozapadna padina, UTM DP02, Exp. SW (leg. Pavlović, Z. 10.04.1948., det. Pavlović, Z., BEO 1011).

\section{Pinus mugo Turra subsp. mugo}

M Prokletije: Mokra gora, UTM DN64 (leg. Rudski, I., 16.07.1932., det. Rudski, I., BEO 994); Pogled, Crvene vode, UTM DN43 (leg. Rudski, I. 03.07.1932., det. Rudski, I., BEO 998); Mokra planina, Mirkove Vode, UTM DN54 (leg. Rudski, I. 04.07.1932., det. Rudski, I., BEO 1004); Radopolje, UTM DN44 (leg. Rudski, I. 17.07.1932., det. Rudski, I., BEO 996); Nedžinat, UTM DN31 (leg. Černjavski, P., Rudski, I., Lindtner, V. 29.07.1933., det. Černjavski, P., BEO 1000); Nedžinatsko jezero, UTM DN31 (leg. Černjavski, P., Rudski, I., Lindtner, V. 28.07.1933., det. Černjavski, P., BEO 1002); vrh, UTM DN31, Mughetum (leg. Černjavski, P., Rudski, I., Lindtner, V. 28.07.1933., det. Černjavski, P., BEO 1001); Žljeb, UTM DN33, krečnjak, kamenjari u šumi molike (leg. Nikolić, V., Diklić, N., Mladenović, S. 20.07.1980., det. Sigunov, A., BEO 35772).

Pinus nigra J. F. Arnold subsp. pallasiana (Lamb.) Holmboe

M Prokletije: Koprivnik, UTM DN31 (leg. Černjavski, P., 08.1934., det. Černjavski, P., BEO 1062).

\section{TAXACEAE}

\section{Taxus baccata L.}

Bt Kikinda: gradski park, UTM DR57 (leg. Izmajlov, N., 21.06.1924., det. Izmajlov, N., BEO 735); M Prokletije: Peć, UTM DN42 (leg. Sobrijevski, 1933., det. Černjavski, P., BEO 728); 
SI Đerdap: Pecka bara - Trajanov put, pored Dunava, UTM FQ03 (leg. Diklić, N., 20.10.1965., det. Diklić, N., BEO 35683); Stol (Bor): zapadna padina, UTM EP99, bukov šibljak (leg. Nikolić, $v$. 24.05.1955., det. Nikolić, V., BEO 35682).

\section{Liliopsida}

\section{ACORACEAE}

\section{Acorus calamus L.}

Bt Deliblatska peščara: Deliblato, UTM EQ06 (leg. Soška, T., 13.07.1943., det. Soška, T., BEO 35160).

\section{HYDROCHARITACEAE}

\section{Stratiotes aloides L.}

Sr Obrež: Obedska bara, UTM DQ15, Phragmitetum communis (leg. Sigunov, A., 02.10.1969., det. Sigunov, A., BEO 80280).

\section{LILIACEAE (AMARYLLIDACEAE)}

\section{Galanthus nivalis L.}

Bt Deliblatska peščara: Šušara, UTM EQ17 (leg. Diklić, N., 21.03.1973., det. Diklić, N., BEO s.n.); $\underline{\mathbf{C}}$ Kopaonik, UTM DN79, smrčeva šuma (leg. Rudski, I., 31.05.1938., det. Rudski, I., BEO 28874); Kraljevo: Stolovi, Dobre strane (vrh), UTM DP62 (leg. Slavković, Ž. 03.1970., det. Diklić, N., BEO s.n.); I Ozren: Janior, UTM EP73, šibljak, Carpinetum orientalis (leg. Diklić, N., 19.04.1957., det. Diklić, N., BEO s.n.); K Sandžak: Ožlje, podnožje, UTM DN56 (leg. Lindtner, V., 16.04.1949., det. Lindtner, V., BEO 28868); SI Đerdap: Golubac, kamenolom, UTM EQ44, bukova šuma, Carpinetum orientalis (leg. Nikolić, V., Diklić, N., 16.04.1968., det. Nikolić, V., BEO s.n.); Miroč, Veliki Štrbac (vrh), UTM FQ03 (leg. Nikolić, V., Diklić, N. 25.04.1968., det. Nikolić, V., BEO s.n.); Majdanpek: Rajkovo-Zaton, UTM EQ72 (leg. Sigunov, A. 03.04.1959., det. Sigunov, A., BEO 82223); Starica, greben, UTM EQ72, kamenjar, Syringetum (leg. Sigunov, A. 31.03.1959., det. Sigunov, A., BEO 82238); Rtanj: rudnik Rtanj, UTM EP74 (leg. Grebenščikov, O. 07.04.1939., det. Grebenščikov, O., BEO 28870); Sr Inđija: Čortanovci, UTM DR20 (leg. Grebenščikov, O., 16.04.1947., det.

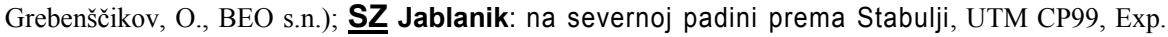
N, bukova šuma (leg. Nikolić, V., 11.05.1955., det. Nikolić, V., BEO s.n.); severna padina, UTM CP99, Exp. NW, bukova šuma (leg. Diklić, N. 25.04.1955., det. Diklić, N., BEO s.n.); vrh, UTM CP99, Exp. NNE, livada (leg. Diklić, N. 24.04.1955., det. Diklić, N., BEO s.n.); planinarski dom, severna padina, UTM CP99, Exp. N, bukova šuma (leg. Nikolić, V., Diklić, N. 25.05.1954., det. Diklić, N., BEO s.n.); Stabulja, pored puta, UTM CP99, bukova šuma (leg. Nikolić, V., Diklić, N. 26.05.1954., det. Diklić, N., BEO s.n.); Maljen: Ribnica, UTM DP29, Quercetum montanum (leg. Sigunov, A. 19.03.1972., det. Sigunov, A., BEO 81935); Medvednik: južna padina, UTM CP99, Exp. S, bukova šuma (leg. Diklić, N. 26.04.1955., det. Diklić, N., BEO s.n.); plato na vrhu, UTM CP99, bukova šuma (leg. Nikolić, V. 11.05.1955., det. Nikolić, V., BEO s.n.); Povlen: Mravinci, Bolje, UTM DP08, krečnjak, Fagetum montanum (leg. Sigunov, A. 20.03.1972., det. Sigunov, A., BEO 82605); Šabac: Jelenovačka šuma, 


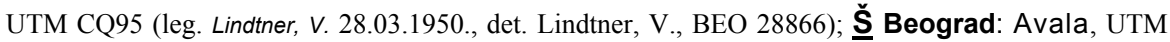
DQ64 (leg. Černjavski, P., 20.03.1938., det. Černjavski, P., BEO s.n.); (leg. Grebenščikov, O. 16.04.1940., det. Grebenščikov, O., BEO); blizu groba neznanog junaka, UTM DQ64 (leg. Mišić, V. 27.03.1949., det. Mišić, V., BEO s.n.); blizu planinske kuće, UTM DQ64 (leg. Mišić, V. 27.04.1949., det. Mišić, V., BEO s.n.); Košutnjak, UTM DQ55 (leg. Grebenščikov, O. 22.03.1940., det. Grebenščikov, O., BEO s.n.); (leg. Černjavski, P. 03.1938., det. Černjavski, P., BEO); blizu Rakovice, UTM DQ55 (leg. Lindtner, V. 03.1940., det. Lindtner, V., BEO s.n.); iznad stanice, UTM DQ55, Tilietum (leg. Grebenščikov, O. 09.03.1933., det. Grebenščikov, O., BEO s.n.); Resnik, UTM DQ55 (leg. Rajevski, L. 09.03.1937., det. Rajevski, L., BEO s.n.); Rušanj, Devojački grob - Petrov grob, UTM DQ54, listopadna šuma (Fagus) (leg. Lindtner, V. 31.03.1954., det. Lindtner, V., BEO s.n.); Gledićke planine: Sahar, vrh, UTM DP95, bukova šuma (leg. Rudski, I. 26.04.1946., det. Rudski, I., BEO 28871); Kragujevac: Karaman, UTM DN89, grabova šuma (leg. Rudski, I. 15.02.1935., det. Rudski, I., BEO 28872); Z Ovčar: Banjski potok, UTM DP36, krečnjak, Fagetum montanum (leg. Sigunov, A., 25.04.1975., det. Sigunov, A., BEO 81119).

\section{Leucojum aestivum L. subsp. aestivum}

Bt Bela Crkva, UTM EQ37 (leg. Izmajlov, N., det. Izmajlov, N., BEO 28885); Pančevo: okolina, UTM DQ76 (leg. Grebenščikov, O. 20.05.1947., det. Grebenščikov, O., BEO s.n.); PančevoOvča, UTM DQ76, vlažne livade (leg. Grebenščikov, O. 20.05.1947., det. Grebenščikov, O., BEO s.n.); JZ Sava: Beograd, UTM DQ56 (leg. Lindtner, V., 12.05.1939., det. Lindtner, V., BEO s.n.); K Uroševac: okolina, UTM EM19, vlažne livade (leg. Jurišić, Ž., 05.1914., det. Jurišić, Ž., BEO 28888); SI Đerdap: Brnjica, UTM EQ64 (leg. Nikolić, V., Diklić, N., 20.04.1968., det. Nikolić, V., BEO s.n.); Pecka bara, UTM FQ04 (leg. Nikolić, V., Diklić, N. 24.04.1968., det. Nikolić, V., BEO s.n.); Negotin: okolina, UTM FP29 (leg. Jovanović, A. 1908., det. Jovanović, A., BEO 28889); SZ Suvobor: Suvobor-Ljig, UTM DP38, Quercetum roboris (leg. Sigunov, A., 20.04.1969., det.

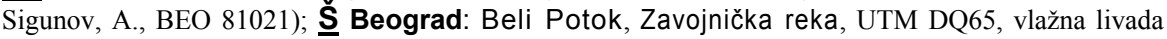
(leg. Diklić, N., 26.04.1954., det. Nikolić, V., BEO s.n.); Kijevo, stanica Kijevo, UTM DQ55 (leg. Grebenščikov, O. 02.05.1933., det. Grebenščikov, O., BEO s.n.); Makiš, UTM DQ55, livade (leg. Krstić, T. 21.04.1953., det. Krstić, T., BEO s.n.); Bele vode, UTM DQ55, vlažne livade (leg. Rudski, I. 10.04.1937., det. Rudski, I., BEO s.n.); Lazarevac: Kolubara, dolina, UTM DQ41, vlažna hrastova šuma (leg. Sigunov, A. 04.04.1973., det. Diklić, N., BEO s.n.).

\section{LILIACEAE (ASPARAGACEAE)}

\section{Prospero autumnale (L.) Speta}

C Prokuplje: Oblačinsko jezero, Oblačinsko polje, UTM EN59 (leg. Adamović, Ž., 19.08. 1973., det. Diklić, N., BEO s.n., sub Scilla autumnalis); 저 Bujanovac: Kraljeva kuća, UTM EN60, mlada sastojina Quercus robur (leg. Diklić, N., 21.09.1961., det. Diklić, N., BEO s.n., sub Scilla autumnalis); Trnovac (s.), iznad sela, UTM EN60, Quercetum confertae-cerris (leg. Diklić, N. 23.09.1961., det. Diklić, N., BEO s.n., sub Scilla autumnalis); Vranje: Zlatokop, pored puta Vranjska Banja, UTM EN70, utrine (leg. Diklić, N. 17.09.1960., det. Diklić, N., BEO s.n., sub Scilla autumnalis).

\section{Ruscus aculeatus L.}

Bt Vršačke planine: Vršački breg, planinarski dom - Kula, UTM EQ29, hrastova šuma (leg. Nikolić, V., Diklić, N., 26.04.1968., det. Diklić, N., BEO s.n.); I Ozren: Janior, UTM EP73, šibljak (leg. Diklić, N., 22.08.1957., det. Diklić, N., BEO s.n.); Po Smederevska Palanka: Mikulja, UTM DQ91, hrastova šuma (leg. Pečnik, 02.06.1943., det. Černjavski, P., BEO 28450); SI Đerdap: potok iza kamenoloma prema Grebenu, UTM EQ83, Exp. NE-SW, šuma graba (leg. Nikolić, V., Diklić, N., 
Rakin, M., 13.09.1965., det. Nikolić, V., BEO s.n.); Donji Milanovac - Greben, sedlo u gornjem delu drugog potoka, UTM EQ92, Exp. NE, šuma sladuna i cera (leg. Nikolić, V., Diklić, N., Rakin, M. 13.09.1965., det. Diklić, N., BEO s.n.); Greben, severoistočne padine prema Dunavu, UTM EQ83, šuma grabića (leg. Nikolić, V., Diklić, N., Rakin, M. 18.09.1965., det. Nikolić, V., BEO s.n.); Greben - Boljetinska reka, UTM EQ83, mešovita šuma (leg. Diklić, N. 18.05.1967., det. Diklić, N., BEO s.n.); Miroč, Veliki Štrbac, UTM FQ03, mešovita šuma (leg. Diklić, N. 16.10.1963., det. Diklić, N., BEO s.n.); Pecka bara, kod Ploča, UTM FQ04 (leg. Nikolić, V., Diklić, N. 25.09.1967., det. Diklić, N., BEO s.n.); Pesača potok, UTM EQ73, bukova šuma (leg. Nikolić, V., Diklić, N. 17.05.1967., det. Diklić, N., BEO s.n.); Poreč-Greben, pored puta, UTM EQ92, mešovita šuma (leg. Nikolić, $v$. 02.08.1966., det. Nikolić, V., BEO s.n.); Miroč: Konjska glava, jugoistočna padina, UTM FQ03,

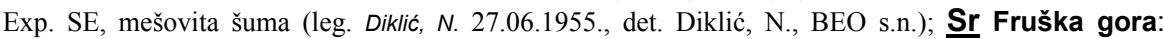
Stražilovo, UTM DR10, šuma (Quercus sessilis-Carpinus orientalis) (leg. Nikolić, V., Diklić, N., 29.05. 1968., det. Diklić, N., BEO s.n.); $\underline{\mathbf{S}}$ Aranđelovac: Risovača, okolina pećine, UTM DQ60, $213 \mathrm{~m}$, krečnjak (leg. Buzurović, U., 01.11.2018., det. Buzurović, U., BEO s.n.); Beograd: Avala, UTM DQ64, hrastova šuma (leg. Đelfi, B. 18.10.1936., det. Đelfi, B., BEO s.n.); borova šuma (leg. Nikolić, V. 14.04.1953., det. Nikolić, V., BEO); Košutnjak, UTM DQ55 (leg. Černjavski, P. 20.07.1943., det. Černjavski, P., BEO s.n.); (leg. Melentijević, Dj. 09.06.1931., det. Melentijević, Dj., BEO); (leg. Đelfi, B. 20.10.1936., det. Đelfi, B., BEO); (leg. Krstić, T. 03.04.1953., det. Krstić, T., BEO); u blizini spomenika knez Mihailo, UTM DQ55, mešovita sastojina (leg. Rudski, I. 11.03.1948., det. Rudski, I., BEO s.n.); Ostružnica, UTM DQ45, hrastova šuma (leg. Krstić, T. 01.10.1953., det. Krstić, T., BEO s.n.).

\section{Ruscus hypoglossum $\mathrm{L}$.}

Bt Vršačke planine: Vršački breg, planinarski dom - Lisičji vrh, UTM EQ39 (leg. Nikolić, V., Diklić, N., 20.06.1969., det. Diklić, N., BEO s.n.); Široko bilo - Lisičja glava, UTM EQ29, šume

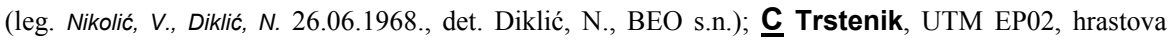
šuma (Quercus conferta) (leg. Černjavski, P., 15.09.1947., det. Černjavski, P., BEO 28454); Veliki Jastrebac: zapadne padine, UTM EP30, Exp. W, bukova šuma (leg. Diklić, N. 20.06.1959., det. Diklić, N., BEO s.n.); Stogorački jelak, UTM EP40, Exp. NW, bukovo-jelova šuma (leg. Diklić, N. 22.07.1959., det. Diklić, N., BEO s.n.); Velika Đulica, UTM EP30 (leg. Sigunov, A. 21.07.1949., det. Krstić, T., BEO 28459); SI Bor: Stol, UTM EP99, Exp. N, bukova šuma (leg. Nikolić, V., Diklić, N., 29.07.1962., det. Diklić, N., BEO s.n.); Crni vrh, UTM EP78, Exp. N, bukova šuma (leg. Nikolić, V. 28.06.1955., det. Nikolić, V., BEO s.n.); Đerdap: Krš, podnožje pored Golubinjske reke, UTM FQ14, EQ73, bukova šuma (leg. Diklić, N. 20.05.1955., det. Diklić, N., BEO s.n.); Veliki Greben, Kroku šerpe, UTM EQ83, bukova šuma (leg. Nikolić, v. 21.06.1956., det. Nikolić, V., BEO s.n.); Majdanpek: Majdanpečka domena, potok Seoski greben, UTM EQ71 (leg. Černjavski, P. 02.08.1946., det. Černjavski, P., BEO 28455); Miroč: Konjska glava, podnožje jugoistočne padine, UTM FQ03, Exp. SE, mešovita šuma (leg. Diklić, N. 27.06.1955., det. Diklić, N., BEO s.n.); Rtanj: severna padina, UTM EP74, Exp. N, bukova šuma (leg. Nikolić, V., Diklić, N., Bogdanović, M. 20.06.1972., det. Diklić, N., BEO s.n.); Sr Fruška gora: Iriški venac, UTM DR00, šuma graba i lipe (leg. Nikolić, V., Diklić, N., 26.05.1970., det. Diklić, N., BEO s.n.); bukova šuma (leg. Nikolić, V., Diklić, N. 27.05.1968., det. Diklić, N., BEO); Iriški venac - Zmajevac, UTM DR00, Exp. N, Fagus-TiliaCarpinus (leg. Nikolić, V., Diklić, N. 09.09.1969., det. Diklić, N., BEO s.n.); Exp. N, bukova šuma (leg. Nikolić, V., Diklić, N. 28.05.1968., det. Diklić, N., BEO); SZ Krupanj: Košutnja stopa, Ćumurana ispod Košutnje stope, UTM CQ63 (leg. Jurišić, Ž., 06.06.1912., det. Jurišić, Ž., BEO 28453); Loznica: Kestebar, Kostenovo, UTM CQ53 (leg. Jurišić, Ž. 03.06.1912., det. Jurišić, Ž., BEO 28456); Š Beograd: Avala, UTM DQ64 (leg. Jurišić, Ž., 01.03.1920., det. Jurišić, Ž., BEO s.n.); Košutnjak, UTM DQ55 (leg. Momčilović, R. 09.12.1979., det. Momčilović, R., BEO s.n.); Lipovica, UTM DQ54 (leg. Černjavski, P. 16.06.1936., det. Černjavski, P., BEO s.n.); blizu Sremčice, UTM DQ54 (leg. Grebenščikov, O. 03.1935., det. Grebenščikov, O., BEO s.n.); Kragujevac: Šumarice, UTM DP84 (leg. Rudski, I. 02.07.1940., det. Krstić, T., BEO 28457). 


\section{LILIACEAE (LILIACEAE)}

\section{Fritillaria meleagris L.}

JI Babušnica: Mlave-Morava, ponor, UTM FN16 (leg. Jurišić, Ž., det. Jurišić, Ž., BEO 28153); Po Smederevska Palanka, UTM DQ91 (leg. Černjavski, P., 1943., det. Černjavski, P., BEO 28152); Ćerovačka Alija, UTM DQ91 (leg. Ranković, B. 01.05.1949., det. Lindtner, V., BEO 28142); Š Lazarevac: Kolubara, UTM DQ31, vlažna hrastova šuma (leg. Sigunov, A., 08.04.1973., det. Diklić, N., BEO 39668); Šopić, UTM DQ41 (leg. X. U. 12.04.1953., det. X. U., BEO 28154).

\section{Fritillaria montana Hoppe ex W. D. J. Koch}

Bt Deliblatska peščara, UTM EQ07 (leg. Grebenščikov, O., 04.1947., det. Grebenščikov, O., BEO s.n., sub F. degeniana); Deliblato, UTM EQ06 (leg. Jurišić, J. 08.05.1922., det. Jurišić, J., BEO 28175, sub F. degeniana); (leg. Grebenščikov, O. 06.04.1945., det. Tomović, G., BEO s.n.); Devojački bunar, UTM DQ98, mešovita listopadna šuma i šikara (leg. Diklić, N. 04.04.1970., det. Diklić, N., BEO 39679, sub F. degeniana); Devojački bunar - Flamunda, UTM EQ08 (leg. Diklić, N. 03.04.1970., det. Diklić, N., BEO 39680, sub F. degeniana); pesak (leg. Nikolić, V., Diklić, N. 26.04.1961., det. Diklić, N., BEO 39681, sub F. degeniana); (leg. Nikolić, V., Diklí, N. 29.04.1968., det. Diklić, N., BEO 39682, sub F. degeniana); SI Đerdap: Pecka bara, iznad Ploča, UTM FQ04 (leg. Dimić, A., 29.04.1967., det. Diklić, N., BEO 39674, sub F. tenella, rev. Buzurović, U. 05.03.2019.); Veliki Štrbac, UTM FQ03 (leg. Nikolić, V., Diklić, N. 25.02.1968., det. Nikolić, V., BEO 39676, sub F. tenella, rev. Buzurović, U. 05.03.2019.).

Lilium carniolicum Bernh. ex W. D. J. Koch subsp. jankae (A. Kern.) Asch. \& Graebn.

I Ruj planina: Zvonce, UTM FN25, livada (leg. Nikolić, V., Diklić, N., 18.06.1966., det. Diklić, N., BEO s.n.); Stara planina, UTM FP30 (leg. Grebenščikov, O. 06.07.1946., det. Grebenščikov, O., BEO 28127); Babin zub, UTM FP30, pašnjaci i kamenjari (leg. Nikolić, V., Diklić, N. 27.06.1975., det. Diklić, N., BEO s.n.); Dobro jutro - Mala poljana, UTM FN58 (leg. Jurišić, Ž. 06.1912., det. Jurišić, Ž., BEO 28118); Jelivičke livade - Prelaz-Vrtop, UTM FN38 (leg. Jurišić, Ž. 06.1912., det. Jurišić, Ž., BEO 28117); Ljuberski rid, UTM FP30, bukovo-jelova šuma (leg. Nikolić, V., Diklić, N. 25.06.1958., det. Nikolić, V., BEO s.n.); Topli Do, Bratkova strana, UTM FN49 (leg. Černjavski, P. 06.07.1946., det. Černjavski, P., BEO 28116); Toplodolska reka, gornji tok, UTM FN39 (leg. Nikolić, V., Diklić, N. 25.06.1958., det. Nikolić, V., BEO s.n.); Zuberski rt, padina Rišora, UTM FP02, livada (leg. Nikolić, v. 25.06.1958., det. Nikolić, V., BEO s.n.); Ženski vrh, UTM FP30, livade (leg. Diklić, N. 21.06.1958., det. Nikolić, V., BEO s.n.); Suva planina, UTM EN98 (leg. Jurišić, Ž. 06.07.1911., det. Jurišić, Ž., BEO 28122); Svrljiške planine: Zeleni vrh, podnožje, UTM FN09, Exp. N, kamenjari (leg. Nikolić, V. 28.06.1957., det. Nikolić, V., BEO s.n.); Vidlič: Basarski kamen, UTM FN38, livade (leg. Nikolić, V., Diklić, N., Lindtner, V. 28.06.1958., det. Nikolić, V., BEO s.n.); Planinica - Mečji vrh, UTM FN38, krečnjak, kamenjari i livade (leg. Diklić, N. 18.06.1974., det. Diklić, N., BEO s.n.); Zvonce: Ranila, Nilhov del, UTM FN25, livade (leg. Nikolić, V., Diklić, N., Rakin, M. 22.07.1964., det. Diklić, N., BEO s.n.); JI Besna kobila, UTM FN00 (leg. llić, Đ., 06.1911., det. Ilić, Đ., BEO 28121, sub L. albanicum, rev. Buzurović, U. 05.03.2019.); ispod vrha, UTM FN00, livade (leg. Nikolić, V., Diklić, N. 22.07.1967., det. Diklić, N., BEO s.n.); Vardenik: Veliki Strešer, UTM FN02, Exp. W, 1700 m, livade (leg. Nikolić, V., Diklić, N. 23.07.1967., det. Diklić, N., BEO s.n.); M Prokletije: Mokra gora, Džora, Seunovo, UTM DN43 (leg. Rudski, I., 28.06.1932., det. Krstić, T., BEO 28123).

\section{Lilium martagon L.}

C Kopaonik: Srebrenac, UTM DN89, livade (leg. Nikolić, V., Diklić, N., Mladenović, S., 29.07. 1979., det. Diklić, N., BEO s.n.); Veliki Jastrebac: Tri sestrice, UTM EP40, kamenjar (leg. Diklić, N. 25.07.1959., det. Diklić, N., BEO s.n.); I Ozren: Leskovik, UTM EP62, kamenjar (leg. Diklić, N., 
26.06.1957., det. Diklić, N., BEO s.n.); Lisičji vrh, UTM DN08, šibljak, Carpinetum orientalis (leg. Diklić, N. 28.06.1957., det. Diklić, N., BEO s.n.); Suva planina: prema Tremu, UTM EN98, krečnjak, kamenjari (leg. Nikolić, V., Diklić, N., Mladenović, S. 26.07.1977., det. Diklić, N., BEO s.n.); Svrljiške planine: Goli vrh, severna padina, UTM FN09, Exp. N, kamenjar (leg. Nikolić, V. 25.07.1957., det. Nikolić, V., BEO s.n.); Pleš, severna padina, UTM EP90, Exp. N, bukova šuma (leg. Nikolić, $V$. 27.07.1957., det. Nikolić, V., BEO s.n.); Zvonačka Banja: Drenjči, UTM FN35, livade (leg. Nikolić, V., Diklić, N., Rakin, M. 19.07.1964., det. Diklić, N., BEO s.n.); severno od banje, UTM FN35, livada (leg. Nikolić, V., Diklić, N., Rakin, M. 15.07.1965., det. Diklić, N., BEO s.n.); J Binačka Morava: Končulj, desna obala, UTM EN50, hrastova šuma, Querceto confertae-cerris (leg. Nikolić, V., 25.06.1966., det. Nikolić, V., BEO s.n.); JZ Nova Varoš: Akmačići, UTM DP00, kamenjari (leg. Nikolić, V., Diklić, N., 10.07.1977., det. Diklić, N., BEO s.n.); Novi Pazar: Sopoćani-Tutin, UTM DN47 (leg. Diklić, N. 14.07.1981., det. Diklić, N., BEO s.n.); Zlatar: Vodena poljana - Golo brdo, UTM DP00, smrčeva šuma (leg. Nikolić, V., Diklić, N. 16.07.1971., det. Diklić, N., BEO s.n.); K Priština: Žegovac, UTM DN91 (leg. Nikolić, V., det. Nikolić, V., BEO s.n.); Šar-planina: Dragaš-Restelica, UTM DM75, ivica jelovih šuma i kamenjari (leg. Nikolić, V., Diklić, N., Mladenović, S. 25.07.1978., det. Diklić, N., BEO s.n.); Jažince-Prevalac, UTM DM97 (leg. Nikolić, V., Diklić, N., Mladenović, S. 20.07.1978., det. Diklić, N., BEO s.n.); M Paštrik: Goropuž, iznad sela, UTM DM67, krečnjak, šuma Quercetum macedonicae i livade (leg. Nikolić, V., Diklić, N., Mladenović, S., 26.07.1979., det. Diklić, N., BEO s.n.); Prizren: Bistrica, klisura, UTM DM77, kamenjar, stene (leg. Nikolić, V., Diklić, N. 13.07.1968., det. Diklić, N., BEO s.n.); Prokletije: Nedžinat, UTM DN31, mešovitoj četinarskoj šumi, zajedno sa Wullfenia (leg. Černjavski, P., Rudski, I., Lindtner, V. 28.07.1933., det. Černjavski, P., BEO 28133); Vrtište, UTM DN54, bukovo-smrčeva šuma (leg. Rudski, I. 15.07.1932., det. Krstić, T., BEO 28134); SI Đerdap: Miroč, Veliki Štrbac (vrh kamene litice iznad Dunava), UTM FQ03, kamene litice (leg. Diklić, N., 22.06.1955., det. Diklić, N., BEO s.n.); Pesača potok, UTM EQ73, bukova šuma (leg. Nikolić, V., Diklić, N. 29.06.1967., det. Diklić, N., BEO s.n.); Kučaj: Malinik, na grebenu, UTM EP76, krečnjak, kamenjar (leg. Nikolić, V., Diklić, N. 27.06.1971., det. Diklić, N., BEO s.n.); Majdanpek: Rajkovo-Zaton, UTM EQ72, Fagetum montanum (leg. Sigunov, A. 03.04.1959., det. Sigunov, A., BEO 82259); Veliki krš: Strelnik, vrh, UTM EP89 (leg. Nikolić, V., Diklić, N., Bogdanović, M. 16.06.1973., det. Diklić, N., BEO s.n.); Sr Fruška gora: Beli kamen, UTM EM69, utrine (leg. Diklić, N., 30.05.1978., det. Diklić, N., BEO s.n.); Iriški venac, UTM DR00, bukova šuma (leg. Nikolić, V., Diklić, N. 27.05.1968., det. Diklić, N., BEO s.n.); put za Čortanovce, UTM DR00 (leg. Nikolić, V., Diklić, N. 27.05.1968., det. Diklić, N., BEO s.n.); Iriški venac - Zmajevac, UTM DR00, Exp. N, bukova šuma (leg. Nikolić, V., Diklić, N. 28.05.1968., det. Diklić, N., BEO s.n.); Stražilovo, UTM DR10, šuma hrasta i lipe (leg. Nikolić, V., Diklić, N. 29.05.1968., det. Diklić, N., BEO s.n.); SZ Jablanik, UTM CP99, bukova šuma (leg. Nikolić, V., Diklić, N., 25.06.1954., det. Nikolić, V., BEO s.n.); severna padina vrha, UTM CP99, Exp. N, livade (leg. Nikolić, V., Diklić, N. 25.05.1954., det. Nikolić, V., BEO s.n.); Stabulja, UTM CP99, livada (leg. Nikolić, V., Diklić, N. 25.07.1954., det. Nikolić, V., BEO s.n.); Valjevo: Poćuta, Stubica, UTM CP98 (leg. Rudski, I. 24.06.1932., det. Rudski, I., BEO

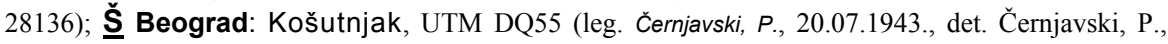
BEO s.n.); Kragujevac: Beloševac, UTM DP97 (leg. Rudski, I. 26.06.1940., det. Rudski, I., BEO 28143); Đuriselo, UTM DP86, livade (leg. Rudski, I. 22.05.1938., det. Rudski, I., BEO 28142); Grošnica, UTM DP96 (leg. Rudski, I. 24.05.1940., det. Krstić, T., BEO 28139); Knić, UTM DP76 (leg. Rudski, I. 16.06.1939., det. Krstić, T., BEO 28140); Merkovac, UTM DP97 (leg. Rudski, I. 22.05.1937., det. Rudski, I., BEO 28144); Rudnik, UTM DP69, Exp. W-SW, Quercetum montanum (leg. Diklić, N. 06.06.1962., det. Diklić, N., BEO s.n.); Z् Ivanjica: Katići, okolina, UTM DP22, livade (leg. Nikolić, V., Mladenović, S., 10.07.1982., det. Diklić, N., BEO s.n.); Studenica, UTM DP16 (leg. Rudski, I. 01.06.1940., det. Krstić, T., BEO 28137); Tara planina: Čemerišta, Ivica-Rastište, UTM CP76 (leg. Jurišić, Ž. 21.06.1912., det. Jurišić, Ž., BEO 28129); Kozja stena, UTM CP76, Exp. SE, krečnjak, Fageto-Pinetum (leg. Sigunov, A. 22.08.1972., det. Sigunov, A., BEO 82151); Perućac, iznad izvora, UTM CP76 (leg. Nikolić, V., Diklić, N., Rakin, M. 13.07.1963., det. Diklić, N., BEO s.n.); 
Rača, UTM CP86, šuma Ostrya carpinifolia (leg. Diklić, N., Rakin, M. 16.07.1963., det. Diklić, N., BEO s.n.); Zajača: Podrinje, UTM CQ60 (leg. Jurišić, Ž. 04.06.1912., det. Jurišić, Ž., BEO 28135).

\section{LILIACEAE (MELANTHIACEAE)}

\section{Veratrum lobelianum Bernh.}

Bt Deliblatska peščara: Deliblato, UTM EQ06 (leg. Soška, T., 1940, det. Soška, T., BEO

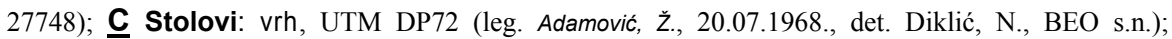
Greda-Usovica, UTM DP62, livade (leg. Nikolić, V., Diklić, N. 12.07.1967., det. Diklić, N., BEO s.n.); I Ruj planina: Cvetkova livada, UTM FN24, livada (leg. Nikolić, V., Diklić, N. 14.07.1965., det. Diklić, N., BEO s.n.); Zvonce, UTM FN25, livade (leg. Nikolić, V., Diklić, N., 18.06.1966., det. Diklić, N., BEO s.n.); Stara planina: Babin zub, UTM FP30, pašnjaci i kamenjari (leg. Nikolić, V., Diklić, $N$. 27.06. 1975., det. Diklić, N., BEO s.n.); Tupanar, podnožje na istočnoj padini, UTM FP30, Exp. E (leg. Nikolić, V., Diklić, N. 23.06.1958., det. Nikolić, V., BEO s.n.); Vidlič: po grebenu, UTM FN48, krečnjak, kamenjari (leg. Nikolić, V., Diklić, N. 16.06.1976., det. Diklić, N., BEO s.n.); JI Besna kobila: planinarski dom, UTM FN00, livade (leg. Nikolić, V., Diklić, N., 22.07.1967., det. Diklić, N., BEO s.n.); Vlasina: Biljene bare, UTM FN13, livada (leg. Černjavski, P. 11.08.1930., det. Černjavski, P., BEO 27761); JZ Golija: Ladne vode, UTM DN49, livade i proplanci u smrčevoj šumi (leg. Nikolić, V., Mladenović, S., 09.07.1982., det. Diklić, N., BEO s.n.); Pešter, UTM DN27, pašnjaci i livade (leg. Nikolić, V., Diklić, N., Mladenović, S. 19.07.1980., det. Diklić, N., BEO s.n.); Sjenica: Pešterska visoravan, Karajukića bunari, UTM DN27, livada (leg. Nikolić, V. 19.03.1966., det. Nikolić, V., BEO s.n.); Zlatar: Vićena poljana - Golo brdo, UTM DP00, livadak (leg. Nikolić, V., Diklić, N. 16.07. 1971., det. Diklić, N., BEO s.n.); K Šar-planina: Ošljak, Prevalac, UTM DM96, livade (leg. Nikolić, V., Diklić, N., 13.07.1968., det. Diklić, N., BEO s.n.); Stojkova kuća - Jezerska čuka, UTM EM06, pašnjaci i kamenjari (leg. Nikolić, V., Diklić, N., Mladenović, S. 20.07.1977., det. Diklić, N., BEO s.n., sub V. album subsp. lobelianum); Stojkova kuća - Piribreg, UTM EM06, pašnjaci i kamenjari (leg. Nikolić, V., Diklić, N., Mladenović, S. 27.07.1976., det. Diklić, N., BEO s.n., sub V. album subsp. lobelianum); M Prokletije: Đeravica - Derviš kom, UTM DN21, pašnjaci i kamenjari (leg. Nikolić, V., Diklić, N., Bogdanović, M., 17.07.1973., det. Diklić, N., BEO s.n.); Šar-planina: Drajčići - Crni vrh, UTM DM96, livade, (leg. Nikolić, V., Diklić, N., Mladenović, S. 17.07.1977., det. Diklić, N., BEO s.n., sub V. album subsp. lobelianum); livade, pašnjaci i kamenjari (leg. Nikolić, V., Diklić, N., Mladenović, S. 18.07. 1978., det. Diklić, N., BEO, sub V. album subsp. lobelianum); SZ Maljen: Divčibare, UTM DP18, Exp. E, serpentinit, močvara, Pinetum sylvestris (leg. Sigunov, A., 22.06.1969., det. Sigunov, A., BEO 81849); Z Tara planina: Mitrovac, kod letovališta, UTM CP76, livada (leg. Nikolić, V., Diklić, N., 13.07.1960., det. Diklić, N., BEO s.n., sub V. album var. viride); Zlatibor: Čigota, UTM DP03, serpentinit, pašnjaci (leg. Nikolić, V., Diklić, N. 12.07.1977., det. Diklić, N., BEO s.n.).

\section{Veratrum nigrum L.}

I Ozren: Leskovik, UTM EP62, kamenjari (leg. Nikolić, V., 24.07.1957., det. Nikolić, V., BEO s.n.); Oštra čuka, UTM EP72, pašnjak (leg. Diklić, N. 25.06.1957., det. Diklić, N., BEO s.n.); Rtanj: istočna padina iznad naselja, UTM EP74, Exp. E (leg. Nikolić, V., Diklić, N. 29.06.1971., det. Diklić, N., BEO s.n.); Suva planina: prema Tremu, UTM EN98, krečnjak, kamenjari (leg. Nikolić, V., Diklić, N., Mladenović, S. 26.07.1977., det. Diklić, N., BEO s.n.); Svrljiške planine: Pleš, UTM EP90, livade (leg. Nikolić, V. 27.07.1957., det. Diklić, N., BEO s.n.); Vlaška planina, UTM FN36, kamenjari (leg. Nikolić, V., Diklić, N., Rakin, M. 21.07.1964., det. Diklić, N., BEO s.n.). 


\title{
Magnoliopsida
}

\section{AMARANTHACEAE (CHENOPODIACEAE)}

\author{
Bassia laniflora (S. G. Gmelin) A. J. Scott
}

Bč Subotičko-horgoška peščara, UTM DS10 (leg. Adamović, Ž., 15.08.1970., det. Diklić, N., BEO 37517, sub Kochia laniflora); Bt Deliblatska peščara: Deliblato, UTM EQ06 (leg. Soška, T., 07.1940., det. Soška, T., BEO 3223, sub Kochia laniflora); (leg. Pichler, A. 1940, det. Soška, T., BEO 3224, sub Kochia arenaria); živi pesak (leg. Miljković, B. 1929., det. Miljković, B., BEO 3225, sub Kochia laniflora); Flamunda, UTM EQ07 (leg. Diklić, N. 09.1968., det. Diklić, N., BEO 37516, sub Kochia laniflora); SI Đerdap: Golubac, Golubački pesak, UTM EQ44 (leg. Adamović, Ž., 27.08. 1965., det. Diklić, N., BEO 37518, sub Kochia laniflora); Prahovo: Radujevac, UTM FQ30 (leg. Jurišić, Ž. 09.07.1912., det. Jurišić, Ž., BEO 3226, sub Kochia arenaria).

\section{Salsola soda L.}

Bt Melenci: Banja Rusanda, UTM DR44 (leg. Tucakov, 09.1969., det. Diklić, N., BEO 37533); Novi Bečej, UTM DR34 (leg. Lindtner, V. 18.06.1955., det. Lindtner, V., BEO 3253).

\section{ARISTOLOCHIACEAE}

\section{Asarum europaeum L. subsp. europaeum}

C Kopaonik: Duboka reka, UTM DN89 (leg. Rudski, I., 05.07.1932., det. Nikolić, V., BEO 5331); Kruševac: Gaglovo, Plandište, UTM CN89, šuma graba i leske. (leg. Bogdanović, $M$. 13.07.1974., det. Nikolić, V., BEO 39484); I Ozren: Lepterija iznad Moravice, UTM EP73, mladi bukovi šumarak (leg. Diklić, N., 21.05.1957., det. Diklić, N., BEO 39492); Oštra čuka, UTM EP72, 1000 m, krečnjak, bukova šuma (leg. Diklić, N., Mladenović, S. 20.07.1988., det. Diklić, N., BEO 39506); JZ Golija: Kušići, severoistočne padine vrha, UTM DP21, Exp. NE (leg. Nikolić, V., Diklić, N., 16.07.1982., det. Diklić, N., BEO 39501); Javor: Kušići, severozapadne padine vrha, UTM DP21, Exp. NW, livade (leg. Nikolić, V., Mladenović, S. 15.07.1982., det. Nikolić, V., BEO 39502); M Prokletije: Mokra planina, Vrelski potok, UTM DN54 (leg. Rudski, I., 06.07.1932., det. Nikolić, V., BEO 5330); Po Smederevska Palanka: Pridvorački lugovi, UTM DQ91 (leg. Banković, B., 01.05.1949., det. Lindtner, V., BEO 5339); SI Crni vrh: zapadna padina, UTM EP78, Exp. W, bukova šuma (leg. Nikolić, V., 27.05.1955., det. Nikolić, V., BEO 39505); Đerdap: Golubac, Golubački grad, UTM EQ54, šuma grabića (leg. Nikolić, V., Diklić, N. 15.05.1967., det. Diklić, N., BEO 39480); Majdanpek: Majdanpečka domena, potok Spinja, UTM EQ71 (leg. Černjavski, P. 19.07.1946., det. Černjavski, P., BEO 5337); Miroč: Mihajlov ponor, UTM FQ04, mešovita šuma (leg. Diklić, N. 27.06.1955., det. Diklić, N., BEO 39495); Veliki Štrbac, blizu vrha, UTM FQ03, Exp. W, 700 m, mešovita šuma (leg. Diklić, N. 20.05.1955., det. Diklić, N., BEO 39494); Visoki čukar, UTM FQ03, Exp. E-NE, mešovita šuma (leg. Diklić, N. 24.05.1955., det. Diklić, N., BEO 39493); Sr Fruška gora: Crveni čot, UTM CR90, mešovite listopadne šume (leg. Nikolić, V., Diklić, N., 06.05.1971., det. Diklić, N., BEO 39487); Irig, UTM DQ19 (leg. Sigunov, A. 20.04.1947., det. Nikolić, V., BEO 39504); Iriški venac, UTM DR00, šuma graba i lipe (leg. Nikolić, V., Diklić, N. 26.05.1970., det. Diklić, N., BEO 39499); spomenik, UTM CR90, mešovita hrastova šuma (leg. Černjavski, $P$.

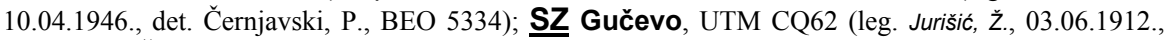
det. Jurišić, Ž., BEO 5338); Jablanik, UTM CP99, Exp. NW, stara bukova šuma (leg. Nikolić, V., Diklić, N. 27.05.1954., det. Diklić, N., BEO 39497); planinarski dom, UTM CP99, Exp. N, bukova šuma (leg. Nikolić, V., Diklić, N. 25.05.1954., det. Diklić, N., BEO 39498); podnožje istočne padine, UTM CP99, Exp. N, ivica bukove šume (leg. Diklić, N. 23.04.1955., det. Diklić, N., BEO 39491); Lajkovac: Man. 
Bogovađa, UTM DQ30, mešovita bukova šuma (leg. Černjavski, P. 07.07.1942., det. Černjavski, P., BEO 5336); Medvednik, UTM DP28, Exp. SE, bukova šuma (leg. Nikolić, V., Diklić, N. 26.05.1954., det.

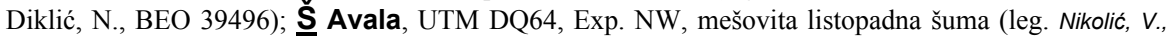
Diklić, N., Mladenović, S., 06.05.1977., det. Nikolić, V., BEO 39503).

\section{BETULACEAE (BETULACEAE)}

\section{Betula pendula Roth}

C Stolovi: Čiker, UTM DP62, Exp. SE, 1300 m, kao ivica bukovika (leg. Jurišić, J., 01.06.1924., det. Jurišić, J., BEO 1161); JI Vlasina: Vlasinska reka, na obali, UTM EN86 (leg. Černjavski, P., 17.08.1930., det. Černjavski, P., BEO 1163); $\underline{M}$ Prokletije: Dečanska planina, UTM DN31, u mešovitoj bukovoj šumi (leg. Černjavski, P., Rudski, I., Lindtner, V., 18.07.1933., det. Černjavski, P., BEO 1154); Man. Visoki Dečani, pored Dečanske Bistrice, UTM DN31 (leg. Černjavski, P., Rudski, I., Lindtner, V. 14.07.1933., det. Černjavski, P., BEO 1159); Mokra gora, Prilate, UTM DN54 (leg. Rudski, I. 15.07.1932., det. Rudski, I., BEO 1155); SI Majdanpek: Debeli Lug, UTM EQ61, šljaka na drumu. (leg. Černjavski, P., 08.1946., det. Černjavski, P., BEO 1168); Majdanpečka domena, Greben Mustafe, sev. padina blizu Grebove reke, UTM EQ71 (leg. Černjavski, P. 03.08.1946., det. Černjavski, P., BEO 1169); Majdanpek, UTM EQ71 (leg. Černjavski, P. 08.1946., det. Černjavski, P., BEO 1167); Mustafa, severni bočni greben, podnožje, UTM EQ71 (leg. Černjavski, P. 10.08.1946.,

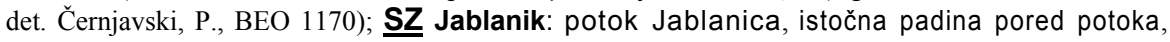
UTM CP99, ivica šume (leg. Nikolić, V., Diklić, N., 28.06.1954., det. Nikolić, V., BEO 1171); Maljen: Divčibare, UTM DP28 (leg. Černjavski, P. 29.09.1946., det. Černjavski, P., BEO 1166); Vlašić, UTM DQ02 (leg. Jurišić, Ž. 24.08.1911., det. Jurišić, Ž., BEO 1152).

\section{BETULACEAE (CORYLACEAE)}

\section{Corylus colurna L.}

I Greben planina, UTM FN35, šibljak (leg. Nikolić, V., Diklić, N., 16.07.1965., det. Diklić, N., BEO 35967); Niš: Koritnik, UTM EN89, šuma grabića (leg. Diklić, N. 03.06.1963., det. Diklić, N., BEO 35969); Ozren: Janior, UTM EP73, šibljak (leg. Diklić, N. 22.07.1960., det. Diklić, N., BEO 35971); Sokobanja, Janior, UTM EP73, šibljak Carpinetum orientalis (leg. Diklić, N. 19.05.1957., det. Nikolić, V., BEO 35975, sub C. colurna f. glandulifera); sanatorijum, UTM EP63 (leg. Diklić, N. 22.07.1960., det. Diklić, N., BEO 35970); Rtanj, UTM EP41, Exp. E, 900 m, sastojina grabića i jorgovana (leg. Diklić, N. 10.06.1965., det. Diklić, N., BEO 35962); Sićevačka klisura, UTM EN89, Exp. S, šuma (leg. Matvejev, S. 30.07.1945., det. Černjavski, P., BEO 1357); Sićevačka klisura (leg. Grebenščikov, O. 14.05.1949., det. Grebenščikov, O., BEO 1358); Kusača, UTM EN99, stene (leg. Nikolić, V., Diklić, N. 26.05.1966., det. Diklić, N., BEO 35958); Ostrvica, Man. Sv. Petka, UTM EN99 (leg. Pavlović, Z. 02.06.1947., det. Diklić, N., BEO 35973); Svrljiške planine: Pernatica, između Pernatice i Pleša, UTM EP90, kamenjar (leg. Nikolić, V. 26.06.1957., det. Nikolić, V., BEO 35979, sub C. colurna f. glandulifera); Tupižnica: zapadna padina, UTM EP94, šibljak (leg. Diklić, N. 23.07. 1962., det. Diklić, N., BEO 35956); Vidlič: Planinica - Basarski kamen, UTM FN38, 1000-1250 m, krečnjak, vrtače i kamenjari (leg. Nikolić, V., Diklić, N. 16.06.1974., det. Nikolić, V., BEO 35963); Zvonačka Banja: severno od banje, UTM FN35, hrastova šuma (leg. Nikolić, V., Diklić, N., Rakin, M.

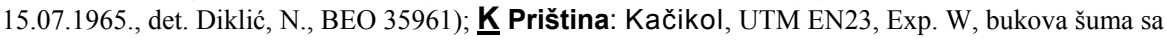
javorom (leg. Nikolić, V., 01.06.1961., det. Nikolić, V., BEO 35978, sub C. colurna f. glandulifera); Zlaš: Orman, UTM EN22, bukovo-grabova šuma sa mečikom leskom (leg. Nikolić, V. 01.06.1961., det. Nikolić, V., BEO 35981, sub C. colurna f. glandulifera); Žegovac: Dronik, UTM DN85, Exp. e, bukova šuma (leg. Nikolić, V. 04.06.1961., det. Nikolić, V., BEO 35980, sub C. colurna f. glandulifera); M Paštrik: Gorožup, UTM DM67, Exp. S, 500-1500 m, krečnjak, šuma Quercetum macedonicaei 
livade (leg. Nikolić, V., Diklić, N., Mladenović, S., 26.07.1979., det. Diklić, N., BEO 35964); Prokletije: Ločanska Bistrica, kod potoka u bukovoj šumi, UTM DN40, bukova šuma (leg. Černjavski, $P$., Rudski, I., Lindtner, V. 15.07.1933., det. Černjavski, P., BEO 1359); SI Đerdap: Pesača potok, UTM EQ73, bukova šuma (leg. Nikolić, V., Diklić, N., 29.06.1967., det. Nikolić, V., BEO 35959); Miroč: Konjska glava, prevoj između Konjska glava i Visokog Čukara, UTM FQ03, mešovita šuma (leg. Diklić, N., Pajović, M. 28.07.1955., det. Diklić, N., BEO 35957); Veliki Štrbac, južna padina glavnog visa, UTM FQ03, Exp. S, 550 m, retka mešovita šuma (leg. Diklić, N. 20.05.1955., det. Diklić, N., BEO 35976, sub C. colurna f. glandulifera); Pecka bara - lugarnica, UTM FQ03, pored puta (leg. Nikolić, V., Diklić, N. 25.06.1967., det. Diklić, N., BEO 35955); Visoki čukar, južna padina, UTM FQ03, Exp. S, 500 m, šibljak (leg. Diklić, N. 18.05.1955., det. Diklić, N., BEO 35977, sub C. colurna f. glandulifera); Stol: Vizjak, UTM EP99, krečnjak, livade i kamenjari (leg. Nikolić, V., Diklić, N. 12.07. 1985., det. Diklić, N., BEO 35974); Veliki krš: Bor, UTM EP89, Exp. SW, šibljak (leg. Diklić, N. 29.06.1960., det. Diklić, N., BEO 35968); Exp. E-NE, bukova šuma (leg. Diklić, N. 22.06.1962., det. Diklić, N., BEO 35972); Strelinik, zapadno podnožje, UTM EP89, Exp. W (leg. Nikolić, V., Diklić, N., Bogdanović, M. 16.06.1973., det. Nikolić, V., BEO 35960); Žagubica: Vrelo Mlave - Buk, UTM EP69, čistine u pojasu bukove šume (leg. Diklić, N. 23.09.1969., det. Diklić, N., BEO 35966).

\section{BRASSICACEAE (CRUCIFERAE)}

\section{Brassica elongata Ehrh. subsp. elongata}

Sr Fruška gora: Čortanovci-Venac, pored puta, UTM DR20, međe (leg. Diklić, N., Mladenović, S., 11.07.1983., det. Jovanović, M., BEO 42323).

\section{Cardamine waldsteinii Dyer}

SZ Jablanik: Novakovići, UTM CP99, Exp. N, bukova šuma pored puta (leg. Nikolić, V., Diklić, N., 27.05.1954., det. Diklić, N., BEO 41406, sub C. savensis); Stabulja, UTM CP99, bukova šuma pored puta (leg. Diklić, N. 26.05.1954., det. Diklić, N., BEO 41403, sub C. savensis); izvor kod planinarske kuće, UTM CP99, bukova šuma oko izvora (leg. Nikolić, V., Diklić, N. 25.05.1954., det. Diklić, N., BEO 41402, sub C. savensis); severozapadna padina, UTM CP99, Exp. N-NW, stara bukova šuma (leg. Nikolić, V., Diklić, N. 22.05.1954., det. Diklić, N., BEO 41400, sub C. savensis); Medvednik: plato na vrhu, UTM CP99, bukova šuma (leg. Nikolić, V. 11.05.1955., det. Nikolić, V., BEO 41405, sub C. savensis); vrh, UTM CP99, proređena bukova šuma (leg. Nikolić, V., Diklić, N. 26.05.1954., det. Diklić, N., BEO 41404, sub C. savensis); $\underline{\mathbf{Z}}$ Tara planina: Bukova glava, UTM CP76, šuma bukve i jele (leg. Diklić, N., Bogdanović, M., 17.05.1974., det. Diklić, N., BEO 41407, sub C. savensis); Mitrovac, UTM CP76, 1200 m, livade (leg. Diklić, N., Bogdanović, M. 17.05.1974., det. Diklić, N., BEO 41408, sub C. savensis).

\section{Euclidium syriacum (L.) R. Br.}

Bč Bač: Bač-Odžaci, UTM CR62, livade. (leg. Nikolić, V., Diklić, N., 27.05.1978., det. Diklić, N., BEO 41760); Čurug, UTM DR23, sušne livade (leg. Nikolić, V., Diklić, N. 08.06.1977., det. Diklić, N., BEO 41759); Bt Banatski Karlovac, UTM EQ08 (leg. Soška, T., 16.05.1943., det. Soška, T., BEO 7107); Novi Bečej: Vian, UTM DR34, ad vian. (leg. Lindtner, V. 01.06.1956., det. Lindtner, V., BEO 7109); Žabalj, UTM DR22, slatine pored puta (leg. Nikolić, V., Diklić, N. 25.05.1973., det. Nikolić, V., BEO 41762).

\section{Hesperis dinarica Beck}

M Prokletije: Mokra planina, UTM DN54 (leg. Rudski, I., 16.07.1932., det. Stevanović, V., BEO 41705); Žljeb, na putu Rožaje-Peć, UTM DN33 (leg. Černjavski, P., Rudski, I., Lindtner, V. 03.08.1933., det. Černjavski, P., BEO 7063); u klisuri, UTM DN33, stene u klisuri (leg. Rudski, I. 
27.06.1932., det. Stevanović, V., BEO 41706); SZ Jablanik: Debelo brdo, pored puta JablanikStubica, UTM CP98, bukova šuma (leg. Nikolić, V., Diklić, N., 25.06.1954., det. Diklić, N., BEO 41701); ispod vrha, UTM CP99, Exp. N, bukova šuma (leg. Nikolić, V., Diklić, N. 30.07.1954., det. Diklić, N., BEO 41702); jugozapadna padina, UTM CP99, Exp. SW, stara bukova šuma (leg. Nikolić, V., Diklić, N. 25.06.1954., det. Diklić, N., BEO 41703); kod planinarskog doma, UTM CP99, livada (leg. Nikolić, V., Diklić, N. 23.06.1954., det. Diklić, N., BEO 41704).

Hesperis matronalis L. subsp. nivea (Baumg.) Kulcz.

I Stara planina, UTM FP30, livada u bukovoj šumi (leg. Nikolić, V., Diklić, N., 25.06.1958., det. Stevanović, V., BEO 41715, sub H. matronalis subsp. candida).

\section{Hesperis sylvestris Crantz subsp. sylvestris}

SI Đerdap: Donji Milanovac - Greben, pored Dunava, UTM EQ92 (leg. Nikolić, V., Diklić,

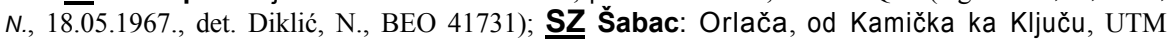
DQ24, šumski i zabareni tereni (leg. Diklić, N., Mladenović, S., 17.05.1988., det. Diklić, N., BEO 41730).

\section{Lepidium cartilagineum (J. Mayer) Thell.}

Bč Subotičko-horgoška peščara: Horgoš, Hajdukovo-Horgoš, UTM DS00, slatine (leg. Nikolić, V., Diklić, N., 07.06.1977., det. Nikolić, V., Diklić, N., BEO 42402); Horgoš-Palić, UTM DS10, močvare i slatine (leg. Diklić, N., Mladenović, S. 23.06.1981., det. Jovanović, M., BEO 42397); KanjižaHorgoš, UTM DS20, utrine (leg. Nikolić, V., Mladenović, S. 28.06.1981., det. Jovanović, M., BEO 42398).

\section{CARYOPHYLLACEAE}

\section{Atocion lerchenfeldianum (Baumg.) M. Popp}

I Stara planina: Belan, Topli Do, UTM FN29, 1200 m, silikat, in rupibus (leg. Grebenščikov, O., 05.07.1946., det. Grebenščikov, O., BEO 4981, sub Silene lerchenfeldiana); K Šar-planina: Bistra prema Prevalacu, severne padine, UTM DM96, Exp. N, 1600-1800 m (leg. Nikolić, V., Diklić, N., 14.07.1968., det. Diklić, N., BEO 39012, sub Silene lerchenfeldiana); M Šar-planina: Dragaš, s. Brod, UTM DM74, Exp. NW (leg. Nikolić, V., Diklić, N., 11.07.1968., det. Nikolić, V., BEO 39013, sub Silene lerchenfeldiana); Draičići - Crni vrh, UTM DM86, DM96, 2000-2600 m, kamenjari (leg. Nikolić, V., Diklić, N. 17.1977., det. Nikolić, V., BEO 39010, sub Silene lerchenfeldiana).

\section{Dianthus monadelphus Vent. subsp. pallens (Sm.) Greuter \& Burdet}

C Prokuplje: Hisar, UTM EN48 (leg. Lindtner, V., 05.07.1953., det. Lindtner, V., BEO 4736, sub D. pallens); I Niš: Gorica, UTM EN79 (leg. llić, Đ., 07.1910., det. Ilić, Đ., BEO 4739, sub D. pallens); Sokobanja, UTM EP63, in vinetis (leg. llić, Đ. 07.1907., det. Ilić, Đ., BEO 4737, sub D. pallens); J Aleksinac: Logorište, UTM EP52, in vinetis (leg. llić, Đ., 08., det. Ilić, Đ., BEO 4738, sub D. pallens).

\section{Dianthus superbus L. subsp. superbus}

I Zvonačka Banja: Rakita, Vilnov del, UTM FN25, livade (leg. Nikolić, V., Diklić, N., Rakin, M., 22.07.1964., det. Diklić, N., BEO 38674); JI Vardenik: Strešer, UTM FN01 (leg. llić, Đ., 08.1910., det. Ilić, Đ., BEO 4750); Vlasina: Stratorija, Stratorija - Duga Zanoga, UTM FN12, livada (leg. Černjavski, P. 15.08.1930., det. Černjavski, P., BEO 4748); Vlasinsko jezero, UTM FN03, $1200 \mathrm{~m}$, livade (leg. Diklić, N. 23.07.1966., det. Diklić, N., BEO 38675); kod hotela, UTM FN03, livade kod 
hotela (leg. Nikolić, V., Diklić, N., Bogdanović, M. 25.07.1972., det. Nikolić, V., BEO 38666); mali most veliki most, UTM EN86, livada (leg. Černjavski, P. 08.08.1930., det. Černjavski, P., BEO 4749); K Šar-planina: Stojkova kuća - Jezerska čuka, UTM EM06, 1200-2000 m, pašnjaci i kamenjari (leg. Nikolić, V., Diklić, N., Mladenović, S., 20.07.1977., det. Nikolić, V., BEO 38671); $\underline{M}$ Šarplanina: Drajčići - Crni vrh, UTM DM96, livade, pašnjaci i kamenjari (leg. Nikolić, V., Diklić, N., Mladenović, S., 18.07.1978., det. Nikolić, V., BEO 38669); Z Zlatibor: Partizanske vode, UTM CP94, livade (leg. Bogdanović, M., 07.1975., det. Nikolić, V., BEO 38676).

\section{Gypsophila paniculata L.}

Bt Deliblatska peščara: Deliblato, UTM EQ06, u sađenoj šumi (leg. Černjavski, P., 02.07. 1927., det. Černjavski, P., BEO 4119); SI Đerdap: Kladovo, UTM FQ24, pesak (leg. Diklić, N., 28.07.1964., det. Diklić, N., BEO 38097).

\section{Herniaria glabra L. subsp. glabra}

C Kraljevo: ispod mosta, UTM DP74, inundov. šljunak ispod mosta (leg. Jurišić, J., 31.05.1924., det. Jurišić, J., BEO 3329); I Stara planina: Topli Do, UTM FN29, in glareosis (leg. Grebenščikov, O., 07.07.1946., det. Grebenščikov, O., BEO 3339); J Binačka Morava: Končuljska klisura, UTM EN50 (leg. Jurišić, Ž., 10.05.1914., det. Jurišić, Ž., BEO 3328); kamenjar, čistine u šumi sladuna i cera (leg. Nikolić, V., Diklić, N. 16.06.1975., det. Nikolić, V., BEO 37574); SI Đerdap: Sip, UTM FQ24, livade (leg. Nikolić, V., Diklić, N., 24.06.1967., det. Nikolić, V., BEO 37573).

\section{Herniaria hirsuta $\mathrm{L}$.}

C Veliki Jastrebac: zapadne padine, UTM EP40, Exp. W, šibljak Carpinetum orientalis (leg. Diklić, N., 23.07.1959., det. Diklić, N., BEO 37553); I Ozren: Sokobanja, UTM DN08, 350 m, solo calc. (leg. Lindtner, V., 07.06.1956., det. Diklić, N., BEO 37554); Vidlič: Basarski kamen, UTM FN38, livade (leg. Lindtner, V., Nikolić, V., Diklić, N. 28.06.1958., det. Diklić, N., BEO 37551); Zvonce: Odorovci, pored puta za selo Odorovci, UTM FN25 (leg. Nikolić, V., Diklić, N. 20.07.1965., det. Nikolić, V., BEO 37550); J Binačka Morava: Končulj, prosek, UTM EN50, prosek, kamenjar (leg. Nikolić, V., 07.06.1967., det. Diklić, N., BEO 38552); Jl klisura Pčinje: Trgovište-Radovnica, UTM FM09, stene (leg. Nikolić, V., Diklić, N., 13.07.1972., det. Nikolić, V., BEO 37549); K Kačanik: Kačanički klanac, UTM EM27 (leg. Jurišić, Ž., 30.04.1914., det. Jurišić, Ž., BEO 3294); Po Stalać: Braljina, Man. Sv. Nikola, UTM EP33, in saxosis (leg. Lindtner, V., 30.06.1953., det. Lindtner, V., BEO 3292).

\section{Moehringia bavarica (L.) Gren.}

M Prokletije: Rugovska klisura, UTM DN32 (leg. Soška, T., 25.06.1925., det. Malý, K., BEO 3768, sub M. malyi var. siparuntia); (leg. Nikolić, V., Diklić, N. 28.07.1971., det. Diklić, N., BEO 37958, sub M. malyi); stene (leg. Nikolić, V., Diklić, N., Bogdanović, M. 14.07.1973., det. Nikolić, V., BEO 37959, sub M. malyi var. siparuntia); Z klisura Rzava: Kršanje, UTM CP75, krečnjak (leg. Diklić, N., Bogdanović, M., 16.05.1974., det. Nikolić, V., BEO 37952, sub M. malyi); Mali Zvornik: okolina, UTM CQ51, stene (leg. Jurišić, Ž. 07.06.1912., det. Jurišić, Ž., BEO 3767, sub M. ponae); Ovčarskokablarska klisura: Ovčar Banja, UTM DP36, krečnjak, stene (leg. Diklić, N., Bogdanović, M. 20.05.1974., det. Nikolić, V., BEO 37955, sub M. malyi); pored pruge, UTM DP36, krečnjak, stene pored pruge (leg. Diklić, N., Mladenović, S. 22.06.1979., det. Diklić, N., BEO 37956, sub M. malyi); Podrinje: Soko tvrđava, UTM CP88 (leg. Jurišić, Ž. 05.06.1912., det. Jurišić, Ž., BEO 3766, sub M. ponae); Tara planina: kanjon Dervente, UTM CP66, 300-350 m, krečnjak, stene (leg. Diklić, N., Bogdanović, M. 18.05.1974., det. Nikolić, V., BEO 37946, sub M. malyi). 


\section{Moehringia pendula (Waldst. \& Kit.) Fenzl}

I Stara planina: Belan, Topli Do, UTM FN29, $1200 \mathrm{~m}$, in rupibus (leg. Grebenščikov, O., 15.07.1946., det. Grebenščikov, O., BEO 3774); Z Rzav: Val ispod Tustog brda, carinarnica mokrogorska, UTM CP92 (leg. Jurišić, Ž., 22.06.1912., det. Jurišić, Ž., BEO 3771); Tara planina: Derventa, UTM CP66 (leg. Jurišić, Ž. 19.06.1912., det. Jurišić, Ž., BEO 3769); Zlatibor: Čajetina, Branište, UTM CP94 (leg. Jurišić, Ž. 25.06.1912., det. Jurišić, Ž., BEO 3770).

\section{Silene viridiflora $\mathrm{L}$.}

C Veliki Jastrebac: prema Orlovom kršu, zapadna padine, UTM EP40, Exp. W, bukova šuma (leg. Diklić, N., 20.07.1959., det. Diklić, N., BEO 39355); I Svrljiške planine: Goli vrh, podnožje, UTM FN09, livada (leg. Nikolić, V., 21.06.1957., det. Nikolić, V., BEO 39357); SI Beljanica: istočna padina, UTM EP58, Exp. E, ivica bukove šume (leg. Diklić, N., 24.09.1969., det. Diklić, N., BEO 39359); Crni vrh, UTM EP78, Exp. S, livada na kosi (leg. Nikolić, V. 27.06.1955., det. Nikolić, V., BEO 39362); Đerdap: Porečka reka, Gradišnica potok, UTM EQ91 (leg. Nikolić, V., Diklić, N. 04.06.1966., det. Nikolić, V., BEO 39354); Veliki Greben, Crni vrh, UTM EQ83, bukova šuma (leg. Nikolić, V. 30.07.1956., det. Nikolić, V., BEO 39358); Stol (Bor): Lučka reka, desna strana, UTM EP88, livade (leg. Nikolić, V., Diklić, N. 28.07.1962., det. Diklić, N., BEO 39356); izvorište, UTM EP99, livada (leg. Nikolić, v. 22.06.1955., det. Nikolić, V., BEO 39361); Z Podrinje: Donja Trešnica, UTM CQ51 (leg. Jurišić, Ž., 06.06.1912., det. Jurišić, Ž., BEO 5149).

Silene viscosa (L.) Pers.

Bt Čoka: Tisa, UTM DR38, slatine (leg. Nikolić, V., Diklić, N., 02.06.1978., det. Nikolić, V., BEO 39366); slatine (leg. Nikolić, V., Diklić, N. 02.06.1978., det. Nikolić, V., BEO); slatine (leg. Nikolić, V., Diklić, N. 02.06.1978., det. Nikolić, V., BEO).

Spergularia media (L.) C. Presl subsp. media

Bt Novi Bečej, UTM DR34 (leg. Lindtner, V., 06.1955., det. Lindtner, V., BEO 3373, sub S. marginata).

\section{CISTACEAE}

Helianthemum salicifolium (L.) Mill.

JI Pčinja: Šajince-Trgovište, UTM EM89, kamenjar (leg. Nikolić, V., Diklić, N., 09.05.1973., det. Nikolić, V., BEO 42613).

Tuberaria guttata (L.) Fourr.

J Binačka Morava: Končuljska klisura, UTM EN50 (leg. Nikolić, V., 29.06.1965., det. Nikolić, V., BEO 42610, sub Helianthemum salicifolium, rev. Zlatković, B. 14.12.2015.).

\section{EUPHORBIACEAE}

\section{Euphorbia carniolica Jacq.}

SI Majdanpek: Majdanpečka domena, potok Dupaš, dno potoka, UTM EQ71 (leg.

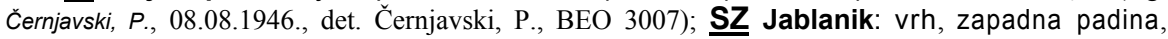


UTM CP99, livada (leg. Nikolić, V., Diklić, N., 25.06.1954., det. Diklić, N., BEO 37266); na severnoj padini kod planinarskog doma, UTM CP99, bukova šuma (leg. Nikolić, V., Diklić, N. 25.05.1954., det. Diklić, N., BEO 37268); Medvednik: jugo-istočna padina, UTM DP28, stara bukova šuma (leg. Nikolić, V., Diklić, N. 26.05.1954., det. Diklić, N., BEO 37267).

\section{Euphorbia lingulata Heuff.}

I Rtanj: jugoistočna padina prema vrhu, UTM EP74, kamenjar (leg. Nikolić, V., Diklić, N., Bogdanović, M., 20.06.1972., det. Nikolić, V., BEO 37241).

\section{FAGACEAE}

\section{Castanea sativa Mill.}

Banjičko brdo, u vinogradu Steve Božovića, vinograd (leg. Jurišić, Ž. 09.09.1911., det. Jurišić,

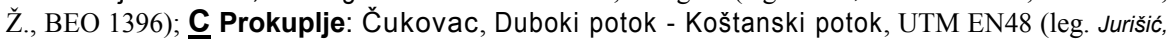
Ž., 01.06.1911., det. Jurišić, Ž., BEO 1398); Pavitnjak - Duboki potok, UTM EN48 (leg. Jurišíc, Ž. 01.06.1911., det. Jurišić, Ž., BEO 1397); I Niš: Gorica, UTM EN79 (leg. Jurišić, Ž., 20.05.1911., det. Jurišić, Ž., BEO 1389); J Leskovac: Hisar, UTM EN48 (leg. Jurišić, Ž., 29.05.1911., det. Jurišić, Ž., BEO 1399); Vranje: Ćoška, UTM EN71 (leg. Jurišić, ž. 23.05.1911., det. Jurišić, Ž., BEO 1401); JI Vladičin Han: Kržinca, UTM EN82, vinograd (leg. Jurišić, Ž., 21.05.1911., det. Jurišić, Ž., BEO 1400); K Kosovska Mitrovica: Orahovo, UTM DN84 (leg. Jurišić, Ž., 01.07.1914., det. Jurišić, Ž., BEO 1380); Šar-planina: Brezovica, Malo borče, UTM DM97, serpentinit, kamenjari (leg. Nikolić, V., Diklić, N., Mladenović, S. 27.07.1976., det. Diklić, N., BEO 36008); M Prokletije: Peć, Gubavac, UTM DN32, šikara sa Forsythia europaea (leg. Nikolić, V., Diklić, N., Bogdanović, M., 16.07.1973., det. Nikolić, V., BEO 36009); Po Svilajnac, UTM EP17, vinograd (leg. Dimitrijević, M., 10.06.1922., det. Jurišić, Ž., BEO 1378); SZ Loznica: Kestenjar, UTM CQ53, kestenjar (leg. Jurišić, Ž., 03.06.1911., det. Jurišić, Ž., BEO 1377); Kličevac, UTM CQ53 (leg. Jurišić, Ž. 03.06.1912., det. Jurišić, Ž., BEO 1391); Vlašić: iznad Crniljeva, UTM DQ02 (leg. Jurišić, Ž. 24.08.1911., det. Jurišić, Ž., BEO 1379); Š Osečina: Lopatanj, u imanju Jovića Blag., UTM CQ80 (leg. Jurišić, Ž., 25.08.1911., det. Jurišić, Ž., BEO 1384); Z Studenica, UTM DP16, sađeno (leg. Rudski, I., 30.06.1932., det. Grebenščikov, O., BEO 1387).

\section{GENTIANACEAE}

\section{Gentiana asclepiadea L.}

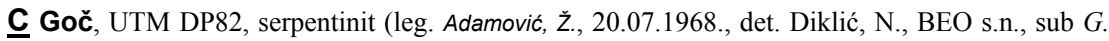
asclepiadea subsp. cruciata); Kopaonik: prema Pančićevom vrhu, UTM DN89, Nardetum strictae (leg. Nikolić, V., Diklić, N., Rakin, M. 18.09.1964., det. Diklić, N., BEO s.n., sub G. asclepiadea subsp. cruciata); Gobelja, UTM DN89, Exp. S, silikat, Juniperetum nanae (leg. Sigunov, A. 24.08.1974., det. Diklić, N., BEO s.n., sub G. asclepiadea subsp. cruciata); Pajino preslo, UTM DN89, vlažne livade u smrčevoj šumi (leg. Diklić, N. 18.08.1982., det. Diklić, N., BEO s.n., sub G. asclepiadea subsp. cruciata); Suvo rudište, UTM DN89 (leg. Sigunov, A. 23.08.1946., det. Sigunov, A., BEO 22045); Exp. NW(leg. Diklić, N. 20.08.1982., det. Diklić, N., BEO s.n., sub G. asclepiadea subsp. cruciata); Kraljevo: Stolovi, Čiker, UTM DP62 (leg. Jurišić, J. 01.06.1924., det. Jurišić, J., BEO 22041); Željin: pašnjak na vrhu, UTM DP81, pašnjak (leg. Adamović, Ž. 20.09.1966., det. Diklić, N., BEO s.n., sub G. asclepiadea subsp. cruciata); I Greben, UTM FN35 (leg. Lindtner, V., 24.07.1933., det. Lindtner, V., BEO 22040); Stara planina: Babin zub, ispod, UTM FP30, Exp. N, iznad smrčeve granice (leg. Lindtner, v. 21.09.1968., det. Lindtner, V., BEO s.n., sub G. asclepiadea subsp. cruciata); Široke Luke, UTM FN58 (leg. Pavlović, P. 23.07.1911., det. Pavlović, P., BEO 22043); Zvonce: Ranila, 
UTM FN25 (leg. Nikolić, V., Diklić, N., Rakin, M. 22.07.1964., det. Diklić, N., BEO s.n., sub G. asclepiadea subsp. pectinata); JI Besna kobila, UTM FN00, livade i pašnjaci (leg. Nikolić, V., Diklić, N., 17.07. 1968., det. Diklić, N., BEO s.n., sub G. asclepiadea subsp. cruciata); JZ Golija, UTM DN49 (leg. Nikolić, V., 03.09.1948., det. Nikolić, V., BEO s.n.); M Prokletije: Žljeb, klisura, UTM DN33 (leg.

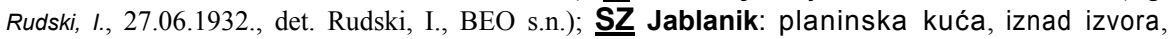
UTM CP99, bukova šuma (leg. Nikolić, V., Diklić, N., 28.07.1954., det. Nikolić, V., BEO s.n.); Stabulja, UTM CP99, bukova šuma (leg. Nikolić, V., Diklić, N. 26.06.1954., det. Nikolić, V., BEO s.n.); Maljen: Divčibare, UTM DP18, Exp. W, Fageto-Abieto-Pinetum (leg. Sigunov, A. 18.09.1969., det. Sigunov, A., BEO 81883); Medvednik, UTM CP99, Exp. S, bukova šuma (leg. Nikolić, V., Diklić, N. 27.06.1954., det. Nikolić, V., BEO s.n.); Valjevo: Poćuta, Stubica, UTM CP98 (leg. Jurišić, Ž. 30.07.1912., det. Jurišić, Ž., BEO 22042); Z Mokra Gora, UTM CP75 (leg. Jurišić, Ž., 22.06.1912., det. Jurišić, Ž., BEO 22051); Tara planina: put prema Kremni, UTM CP85, ivica jelove i smrševe šume (leg. Diklić, $N$. 16.09.1966., det. Diklić, N., BEO s.n., sub G. asclepiadea subsp. pectinata); Rača, kanjon, UTM CP86 (leg. Diklić, N. 18.07.1963., det. Diklić, N., BEO s.n.); Zlatibor: Bela Reka, UTM DP12 (leg. Jurišić, Ž. 19.07.1908., det. Jurišić, Ž., BEO 22050).

\section{Gentiana cruciata L. subsp. cruciata}

I Devica: Gornji Skokar - Koviljak, u vrtači, UTM EP72 (leg. Diklić, N., 24.06.1957., det. Diklić, N., BEO s.n.); Greben, UTM FN35, kamenjar (leg. Nikolić, V., Diklić, N. 17.07.1965., det. Diklić, N., BEO s.n.); klisura Jerme: Vlasi, UTM FN36 (leg. Nikolić, V., Diklić, N., Rakin, M. 18.07.1965., det. Diklić, N., BEO s.n.); Ozren, UTM DN08, livade (leg. Diklić, N. 06.1960., det. Diklić, N., BEO s.n.); Leskovik, na vrhu, UTM EP62, livade (leg. Diklić, N. 26.07.1957., det. Diklić, N., BEO s.n.); Oštra čuka, UTM EP72, pašnjak (leg. Diklić, N. 25.07.1957., det. Diklić, N., BEO s.n.); krečnjak, kamenjari (leg. Diklić, N., Mladenović, S. 20.07.1988., det. Stevanović, V., BEO); Sićevačka klisura: Man. Sv. Petka, UTM EN99 (leg. Bilić, I. 20.07.1945., det. Nikolić, V., BEO 22036); Suva planina: Gornji Dušnik, UTM EN98, šikare i livade (leg. Diklić, N., Mladenović, S. 18.07.1985., det. Stevanović, V., BEO s.n.); Svrljiške planine, UTM FP00, hrastova šuma (leg. Nikolić, V. 24.07.1957., det. Nikolić, V., BEO s.n.); JZ Novi Pazar: Sopoćani-Tutin, UTM DN47 (leg. Nikolić, V., Mladenović, S., 14.07.1981., det. Diklić, N., BEO s.n.); K Šar-planina: Lešnica, UTM DN29 (leg. Pichler, A., 08.1940., det. Pichler, A., BEO s.n.); $\underline{M}$ Šar-planina: Žur-Dragaš, UTM DM76, pojas grabića (leg. Nikolić, V., Diklić, N., 11.07.1969., det. Diklić, N., BEO s.n.); SI Crni vrh, UTM EP78, bukova šuma sa jasikom (leg. Nikolić, V., 01.08.1955., det. Nikolić, V., BEO s.n.); Glavica, kosa od vrha prema Glavici, UTM EP78 (leg. Nikolić, V. 03.08.1955., det. Nikolić, V., BEO s.n.); Đerdap: Tekija, Golo brdo, UTM FQ04 (leg. Nikolić, V., Diklić, N. 18.06.1969., det. Diklić, N., BEO s.n.); Tekija - Pecka bara, Golo brdo, UTM FQ04 (leg. Nikolić, V., Diklić, N. 27.06.1967., det. Diklić, N., BEO s.n.); Rtanj: istočna padina, UTM EP74, Exp. E, krečnjak (leg. Diklić, N., Mladenović, S. 19.07.1988., det. Buzurović, U., BEO s.n.); prema grebenu, UTM EP74, krečnjak (leg. Diklić, N., Mladenović, S. 19.07.1988., det. Buzurović, U., BEO s.n.); SZ Jablanik, UTM CP99, Exp. N, utrina (leg. Nikolić, V., Diklić, N., 27.07.1954., det. Nikolić, V., BEO s.n.); Valjevo: Poćuta, Stubica, UTM CP98 (leg. Jurišić, Ž. 30.07.1912., det. Jurišić, Ž., BEO 22029); Š Kragujevac: Ramaćki visovi, UTM DP78 (leg. Rudski, I., 01.07.1937., det. Rudski, I., BEO 22080); Z Ovčar: Straževina, UTM DP36, Exp. E, Fagetum montanum (leg. Sigunov, A., 22.07. 1976., det. Sigunov, A., BEO 81085); Tara planina: Čemerište, UTM CP76, livada (leg. Nikolić, V., Diklić, N. 15.07.1960., det. Diklić, N., BEO s.n.); Oštre stene, UTM CP76, kamenjari (leg. Nikolić, V., Diklić, N. 14.07.1960., det. Diklić, N., BEO s.n.).

\section{Gentiana lutea L. subsp. symphyandra (Murb.) Hayek}

JZ Priboj: Crni vrh, UTM CP82 (leg. Lindtner, V., 19.07.1949., det. Diklić, N., BEO s.n., sub G. lutea, rev. Buzurović, U. 01.03.2019.); K Šar-planina: Kodža-Balkan (Jezerska planina), UTM EM08, Exp. SE, serpentinit, pašnjaci i kamenjari (leg. Diklić, N., 19.07.1975., det. Diklić, N., BEO s.n.); Lešnica, UTM DN29 (leg. Pichler, A. 08.1940., det. Pichler, A., BEO s.n.); M Paštrik: Goropuž, iznad sela, UTM DM67, krečnjak, šuma Quercetum macedonicae i livade (leg. Nikolić, V., Diklić, N., Mladenović, S., 26.07.1979., det. Stevanović, V., BEO s.n.); Prokletije: Nedžinat, UTM DN31, 
subalpijske i alpijske livade (leg. Rudski, .., Lindtner, V. 28.07.1933., det. Černjavski, P., BEO 22021, sub Gentiana punctata, rev. Buzurović, U. 01.03.2019.); Žljeb, UTM DN33 (leg. Demajo, M. 26.07.1979., det. Buzurović, U., BEO s.n.); $\mathbf{S Z}$ Maljen: Divčibare, UTM DP18, Exp. E, serpentinit, Pinetum sylvestris (leg. Sigunov, A., 01.06.1969., det. Sigunov, A., BEO 80846, sub G. lutea, rev. Buzurović, U. 01.03.2019.); oko vrha, UTM DP18, vlažne livade (leg. Sigunov, A. 15.07.1973., det. Diklić, N., BEO s.n.); Priboj: Crni vrh, UTM CP82 (leg. Nikolić, V. 19.07.1949., det. Nikolić, V., BEO 22015, sub G. lutea, rev. Buzurović, U. 01.03.2019.); Z Maljen: Divčibare, UTM DP18 (leg. Lindtner, V., 07.1948., det. Lindtner, V., BEO 22017, sub G. lutea, rev. Buzurović, U. 01.03.2019.); Zlatibor, UTM CP94, livade, 06.07., BEO 22014); Čigota, UTM DP03, serpentinit, pašnjaci (leg. Nikolić, V., Diklić, N. 12.07.1977., det. Diklić, N., BEO s.n., sub G. lutea, rev. Buzurović, U. 01.03.2019.).

\section{Gentiana punctata L.}

I Stara planina: Midžor, ispod vrha, UTM FP30, Exp. W (leg. Nikolić, V., 23.06.1958., det. Diklić, N., BEO s.n.); Tupanar, UTM FP30, stene (leg. Nikolić, V., Diklić, N. 23.06.1958., det. Diklić, N.,

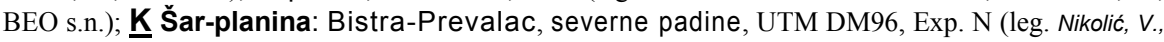
Diklić, N., 14.07.1968., det. Diklić, N., BEO s.n.); Careva livada - Jažinačko jezero, UTM DM96, Exp. NW (leg. Nikolić, V., Diklić, N., Mladenović, S. 24.07.1980., det. Diklić, N., BEO s.n.); Stojkova kuća - Durlov potok, UTM EM06, livade i kamenjari (leg. Nikolić, V., Diklić, N., Mladenović, S. 25.07.1976., det. Diklić, N., BEO s.n.); Stojkova kuća - Jezerska čuka, UTM EM06 (leg. Nikolić, V., Diklić, N., Mladenović, S. 23.07.1980., det. Diklić, N., BEO s.n.); Stojkova kuća - Livadica, UTM EM07, krečnjak, livade i kamenjari (leg. Nikolić, V., Diklić, N. 22.07.1975., det. Diklić, N., BEO s.n.); Stojkova kuća - Piribreg, UTM EM06, kamenjari (leg. Nikolić, V., Diklić, N., Bogdanović, M. 22.07. 1974., det. Diklić, N., BEO s.n.); $\underline{M}$ Prokletije: Derviš kom, UTM DN21, granit (leg. Černjavski, P., Rudski, I., Lindtner, V., 16.07.1933., det. Černjavski, P., BEO 22023); Pločica, UTM DN21 (leg. Lindtner, V. 06.1933., det. Lindtner, V., BEO 22020); Đeravica - Derviš kom, UTM DN21, pašnjaci i kamenjari (leg. Nikolić, V., Diklić, N., Bogdanović, M. 17.07.1973., det. Diklić, N., BEO s.n.); Kurvala, UTM DN31, livade i žbunje (leg. Černjavski, P., Rudski, I., Lindtner, V. 16.07.1933., det. Černjavski, P., BEO 22018); Mokra gora, Radovački stanovi, UTM DN44 (leg. Rudski, I. 29.06.1932., det. Benić, N., BEO s.n.); Pločica - Derviš kom, UTM DN21, granit (leg. Černjavski, P., Rudski, l., Lindtner, V. 17.07.1933., det. Černjavski, P., BEO 22019).

\section{Gentianella ciliata (L.) Borkh. subsp. ciliata}

M Prokletije: Koprivnik, UTM DN31 (leg. Černjavski, P., 08.1934., det. Černjavski, P., BEO 12151); Mokra gora, Međedova leska, UTM DN64, pašnjaci (leg. Nikolić, v. 24.09.1969., det. Diklić, N., BEO s.n.); $\underline{\mathbf{Z}}$ Tara planina: put prema Kremni, UTM CP85, ivica jelove i smrševe šume (leg. Diklić, N., 16.09.1966., det. Diklić, N., BEO s.n.).

\section{GERANIACEAE}

\section{Erodium hoefftianum C. A. Mey. subsp. hoefftianum}

J Aleksinac, UTM EM58, vinogradi (leg. llić, Đ., det. Ilić, Đ., BEO 9531, sub E. tmoleum); Logorište, UTM EP52, vinogradi (leg. llić, Đ. 04., det. Ilić, Đ., BEO 9530); Vranje: okolina, UTM EN71 (leg. llić, Đ. 05.1910., det. Ilić, Đ., BEO 9632).

\section{Geranium macrorrhizum L.}

I Leskovik, UTM EP70, izdanačka bukova šuma (leg. Diklić, N., 22.06.1957., det. Diklić, N., BEO 44093); Sićevačka klisura: Ostrvica, Man. Sv. Petka, UTM EN99 (leg. Grebenščikov, O. 19.05. 1949., det. Diklić, N., BEO 9448); Svrljiške planine: Pleš, UTM EP90, Exp. N, bukova šuma (leg. Nikolić, V. 27.07.1957., det. Nikolić, V., BEO 44090); Tupižnica, UTM EP94, krečnjak (leg. Lindtner, V. 
07.1955., det. Lindtner, V., BEO 9447); Bučje, UTM EP94, 1000-1100 m, krečnjak (leg. Lindtner, V. 22.07.1955., det. Diklić, N., BEO 9445); Zvonačka Banja: tvrđava Kale, UTM FN35 (leg. Tomić, V. 13.08.1955., det. Diklić, N., BEO 44091); Zvonce: Drenjak, vrh, UTM FN36, kamenjar (leg. Nikolić, V., Diklić, N., Rakin, M. 21.07.1964., det. Diklić, N., BEO 44099); J Vranje: Oblik, UTM EN71, 1300 m, bukova šuma (leg. Diklić, N., 01.06.1960., det. Diklić, N., BEO 44092); K Šar-planina: Stojkova kuća - Jezerska čuka, UTM EM06, 1600-2400 m (leg. Nikolić, V., Diklić, N., Mladenović, S., 23.07. 1980., det. Jovanović, M., BEO 44087); M Prokletije: Mokra planina, Crvene vode, UTM DN54, livade u smrčevoj šumi (leg. Rudski, I., 03.07.1932., det. Janković, M., BEO 9459); Debelo brdo, UTM DN69 (leg. Rudski, I. 12.07.1932., det. Janković, M., BEO 9460); Vrelski potok, UTM DN53 (leg. Rudski, I. 02.07.1932., det. Janković, M., BEO 9455); Po Despotovac: klisura Resave, UTM EP57, krečnjak, stene (leg. Nikolić, V., Diklić, N., 12.06.1976., det. Jovanović, M., BEO 44089); SI Đerdap: Pesača potok, UTM EQ73, bukova šuma (leg. Nikolić, V., Diklić, N., 17.05.1967., det. Diklić, N., BEO 44101); Majdanpek: Majdanpečka domena, Veliki Pek, UTM EQ71, u steni (leg. Černjavski, P. 05.08.1946., det. Černjavski, P., BEO 9446); Miroč: Veliki Štrbac, južna padina glavnog visa, UTM FQ03, Exp. S, mešovita šuma (leg. Diklić, N. 22.06.1955., det. Diklić, N., BEO 44100); Velki Štrbac, južna padina glavnog visa, UTM FQ03, Exp. S, 650 m, krečnjak, blokovi stena (leg. Diklić, N. 20.05.1955., det. Diklić, N., BEO 44095); Stol: Vizjak, UTM EP99, kamenjar (leg. Nikolić, V. 06.08.1955., det. Nikolić, V., BEO 44098); vrh, UTM EP99, Exp. W, bukova šuma (leg. Nikolić, V. 25.05.1955., det. Nikolić, V., BEO 44097); Z Studenica: Man. Studenica, UTM DP61 (leg. Rudski, I., 01.06.1940., det. Janković, M., BEO 9450); Tara planina: Derventa, Drum Debo, UTM CP66 (leg. Jurišić, Ž. 19.06.1912., det. Jurišić, Ž., BEO 9453).

\section{Geranium nodosum L.}

Z Tara planina: Derventa, Drum Debo, UTM CP66 (leg. Jurišić, Ž., 19.06.1912., det. Jurišić, Ž., BEO 9416).

\section{Geranium robertianum L. subsp. robertianum}

C Kopaonik: Suvo rudište, UTM DN89 (leg. Rudski, I., 10.1938., det. Rudski, I., BEO 9518); Radan: Petrova gora, kod rasadnika, UTM EN46, bukova šuma (leg. Nikolić, v. 21.07.1959., det. Jovanović, M., BEO 44160); I Ozren: Sokobanja, UTM DN08, 350 m (leg. Lindtner, V., 11.05.1956., det. Diklić, N., BEO 44166); Sićevačka klisura: Ostrvica, Man. Sv. Petka, UTM EN99 (leg. Grebenščikov, O. 14.05.1949., det. Grebenščikov, O., BEO 9514); J Kukavica: Vlajna, vrh, UTM EN73, pašnjak (leg. Nikolić, V., 19.06.1960., det. Jovanović, M., BEO 44161); Jl klisura Pčinje: Man. Prohor Pčinjski, UTM EM78 (leg. Nikolić, V., Diklić, N., 15.05.1975., det. Nikolić, V., BEO 44167); JZ Novi Pazar: Man. Sopoćani, iznad manastira, UTM DN47, šikara sa Ostrya carpinifolia (leg. Diklić, N., Mladenović, S., 13.06.1985., det. Jovanović, M., BEO 44162); SI Crni vrh, UTM EP78, bukova šuma (leg. Nikolić, V., 27.05.1955., det. Nikolić, V., BEO 44163); Đerdap: Donji Milanovac, Varoški potok, UTM EQ82, Exp. NW, 90 m, bukova šuma (leg. Nikolić, V., Diklić, N., Rakin, M. 11.09.1965., det. Nikolić, V., BEO 44169); Pesača potok, UTM EQ73, bukova šuma (leg. Nikolić, V., Diklić, N. 17.05.1967., det. Diklić, N., BEO 44168); Veliki Greben, Crni vrh, UTM EQ83, bukova šuma (leg. Nikolić, V. 30.07.1956., det. Nikolić, V., BEO 44165); Majdanpek: Majdanpečka domena, Valea Fondata, jaruga kod izlaza, UTM EQ71 (leg. Černjavski, P. 21.07.1946., det. Černjavski, P., BEO 9524); Miroč: Krš, Golubinjska reka, UTM EQ92, Exp. S, Syringetum (leg. Diklić, N. 20.06.1955., det. Diklić, N., BEO 44170); Stol: vrh, UTM EP99, bukova šuma (leg. Nikolić, V. 25.05.1955., det. Nikolić, V., BEO 44164); SZ Jablanik: jugozapadna padina, UTM CP99, Exp. SW, proređena bukova šuma, sa strmom kamenitom podlogom (leg. Nikolić, V., Diklić, N., 25.06.1954., det. Nikolić, V., BEO 44171); južna padina, UTM CP99, Exp. S, bukova šuma (leg. Nikolić, V., Diklić, N. 27.05.1954., det. Nikolić, V., BEO 44172); Medvednik: južna padina, UTM DP28, Exp. S, bukova šuma (leg. Nikolić, V., Diklić, N. 27.06.1954., det. Diklić, N., BEO 44173); ̌ㅡ Kragujevac: Knić, UTM DP79 (leg. Rudski, I., 16.06.1939., det. Rudski, I., BEO 9517); Z Rzav: Val ispod Tustog brda, carinarnica mokrogorska, UTM CP92 (leg. Jurišić, Ž., 22.06.1912., det. Jurišić, Ž., BEO 9522); Studenica, UTM DP16 (leg. Rudski, I. 01.06.1940., det. Rudski, I., BEO 9519). 
Geranium subcaulescens L'Hér. ex DC.

K Šar-planina: Brezovica - Stojkova kuća, UTM EM06, kamenjari (leg. Nikolić, V., Diklić, N., Bogdanović, M., 22.07.1974., det. Nikolić, V., BEO 44023, sub G. cinereum subsp. subcaulescens); Stojkova kuća - Jezerska čuka, UTM EM06, 1200-2000 m, pašnjaci i kamenjari (leg. Nikolić, V., Diklić, N., Mladenović, S. 20.07.1977., det. Jovanović, M., BEO 44016, sub G. cinereum subsp. subcaulescens); Stojkova kuća - Piribeg, UTM EM06, 1800-2200 m, krečnjak, kamenjari (leg. Nikolić, V., Diklić, N. 21.07.1975., det. Nikolić, V., BEO 44021, sub G. cinereum subsp. subcaulescens); $\mathbf{M}$ Šarplanina: Draičići - Crni vrh, UTM DM86, DM96, 2000-2600 m, kamenjari (leg. Nikolić, V., Diklić, N., 17.07.1977., det. Jovanović, M., BEO 44017, sub G. cinereum subsp. subcaulescens).

\section{GROSSULARIACEAE}

\section{Ribes alpinum $\mathrm{L}$.}

C Veliki Jastrebac: Pogled, UTM EP30, 1400 m, čistine (leg. Diklić, N., 12.05.1959., det. Diklić, N., BEO 46006).

\section{Ribes uva-crispa L. subsp. uva-crispa}

JZ Zlatar: Vodena poljana - Golo brdo, UTM DP00, 1200-1600 m, smrčeva šuma (leg. Nikolić, V., Diklić, N., 16.07.1971., det. Diklić, N., BEO 46008, sub R. glossularia); M Prokletije: Žljeb, Mokra gora - Savine vode, UTM DN64, 1262 m (leg. Rudski, I., 24.06.1932., det. Nikolić, V., BEO 10794, sub R. glossularia); SI Stol: Bor, UTM EP88, Exp. N, bukova šuma (leg. Nikolić, V., Diklić, N., 27.06.1962., det. Diklić, N., BEO 46010, sub R. glossularia); na putu za Bučje, UTM EP93, Exp. N, bukova šuma (leg. Nikolić, V., Diklić, N. 28.07.1962., det. Diklić, N., BEO 46011, sub R. glossularia); vrh, UTM EP99, bukova šuma (leg. Nikolić, v. 25.05.1955., det. Nikolić, V., BEO 46007, sub R. glossularia).

\section{MALVACEAE (MALVACEAE)}

\section{Althaea officinalis L.}

J Bujanovac, UTM EN60, livade sa vrbom Salix alba (leg. Diklić, N., 21.09.1961., det. Diklić, N., BEO 43749); Po Smederevska Palanka: Popova česma, pored potoka, UTM DQ91 (leg. Ranković, B., 17.08.1947., det. Diklić, N., BEO 43747); SI Đerdap: Ada Poreč, ispod Grebena, UTM EQ80, njive i strnjišta (leg. Nikolić, V., Diklić, N., Rakin, M., 15.09.1965., det. Kosovac, A., BEO 43751); Dobra-Brnjica, pored puta, UTM EQ92, pored puta (leg. Nikolić, V., Diklić, N. 29.09.1966., det. Kosovac, A., BEO 43744); Donji Milanovac - Porečka reka, kulture pored Dunava, UTM EQ92, kulture pored Dunava (leg. Nikolić, V., Diklić, N. 01.08.1966., det. Kosovac, A., BEO 43750); Đervin-Tekija, pored Dunava, UTM FQ14 (leg. Diklić, N. 19.10.1965., det. Kosovac, A., BEO 43745); Porečka reka, ušće, UTM EQ91, strnjišta i kulture pored Dunava, pesak (leg. Nikolić, V., Diklić, N., Rakin, M. 10.09.1965., det. Nikolić, V., Diklić, N., Rakin, M., BEO 43753); Pored puta Tekija - Stari Kovilovski Potok, UTM FQ14 (leg. Diklić, N., Pajović, M. 24.07.1955., det. Diklić, N.,

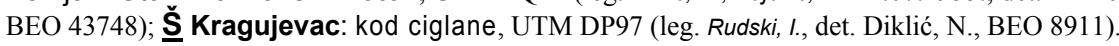

\section{Kitaibelia vitifolia Willd.}

C Lukovo: klisura kod Lukova, UTM EN07, šibljaci (leg. Černjavski, P., 19.07.1939., det. Černjavski, P., BEO 8928); M Prokletije: Koprivnik, UTM DN31 (leg. Černjavski, P., 08.1934., det. Černjavski, P., BEO 8926). 


\section{MALVACEAE (TILIACEAE)}

\section{Tilia cordata Mill. subsp. cordata}

Bt Kikinda, UTM DR57 (leg. Izmajlov, N., 26.07.1922., det. Izmajlov, N., BEO 8986); Vršačke planine: Vršački breg, Široko bilo - Lisičija glava, UTM EQ39, šume (leg. Nikolić, V., Diklić, N. 26.06.1968., det. Diklić, N., BEO 43764); I Stara planina: Temska, Temska - Topli Do, UTM FN28 (leg. Lindtner, V., 22.06.1958., det. Nikolić, V., BEO 43763); $\underline{M}$ Prokletije: Koprivnik, UTM DN31 (leg. Černjavski, P., 08.1934., det. Černjavski, P., BEO 8989); Žljeb, Stubica na putu RožajPeć, UTM DN33, bukova šuma (leg. Černjavski, P., Rudski, l., Lindtner, V. 03.08.1933., det. Černjavski, P., BEO 8983); SI Majdanpek: Majdanpečka domena, potok Spinje, UTM EQ71, bukova sastojina (leg. Černjavski, P., 18.07.1948., det. Černjavski, P., BEO 8982); potok Vrbljani, leva strana, UTM EQ71 (leg. Gajić, M. 11.07.1947., det. Gajić, M., BEO 8985); Veliki Pek, Potkopina, severne padine, UTM EQ71, Exp. N, bukova šuma (leg. Gajić, M. 11.07.1947., det. Gajić, M., BEO 8984); Vučiji Potok, UTM EQ71 (leg. Černjavski, P. 26.07.1946., det. Černjavski, P., BEO 8981); Z Tara planina: Oštre stene, UTM CP76, kamenjar (leg. Nikolić, V., Diklić, N., 14.07.1960., det. Diklić, N., BEO 43766).

\section{Tilia tomentosa Moench}

Bt Deliblatska peščara: Bendin brest, UTM EQ16 (leg. Soška, T., 07.1943., det. Soška, T., BEO 8960); Deliblato, Popara (leg. Soška, T. 26.06.1944., det. Soška, T., BEO 8959); Vršačke planine: Vršački breg, planinarski dom - Kula, UTM EQ29, hrastova šuma (leg. Nikolić, V., Diklić, N. 26.06.1968., det. Diklić, N., BEO 43796); planinarski dom - Lisičiji vrh, UTM EQ39 (leg. Nikolić, V., Diklić, N. 17.07.1969., det. Diklić, N., BEO 43792); I Ozren: Sokobanja, desno od puta za selo Jezero, UTM DN08, livada pored bukove šume (leg. Diklić, N., 02.08.1958., det. Diklić, N., BEO 43798); Janior, UTM EP73, šibljak Carpinetum orientalis (leg. Diklić, N. 19.05.1957., det. Diklić, N., BEO 43800); J Vranje: Vranjska Banja, UTM EN81, Querceto-carpinetum (leg. Diklić, N., 26.09. 1961., det. Diklić, N., BEO 43799); K Gnjilane: Lipovica, UTM EN30, hrastova šuma (leg. Nikolić, V., 24.07.1962., det. Nikolić, V., BEO 43790); $\underline{M}$ Prokletije: Mokra planina, Vrela, UTM DN54 (leg. Rudski, I., 02.07.1932., det. Rudski, I., BEO 8953); SI Đerdap: Donji Milanovac - Greben, Kadin potok, UTM EQ92, 70-100 m, bukova šuma sa orahom (leg. Nikolić, V., Diklić, N., Rakin, M., 14.09.1965., det. Diklić, N., BEO 43789); Greben, severoistočne padine prema Dunavu, UTM EQ83, Exp. NE, šuma grabića (leg. Nikolić, V., Diklić, N., Rakin, M. 18.09.1965., det. Diklić, N., BEO 43802); Greben-Boljetin, pored Dunava, UTM EQ83, mešovita šuma (leg. Nikolić, V., Diklić, N., Rakin, M. 17.09.1965., det. Diklić, N., BEO 43803); Kladovo, Osoj, UTM FQ23, šikara na padinama terase (leg. Adamović, Ž. 06.1968., det. Diklić, N., BEO 43791); Gornjačka klisura: padina sa desne strane reke Mlave, UTM EQ40, kamenjar (leg. Nikolić, V. 11.07.1956., det. Nikolić, V., BEO 43801); Majdanpek: Majdanpečka domena, UTM EQ71, mešovita hrastova sastojina (leg. Černjavski, P. 29.07.1946., det. Černjavski, P., BEO 8933); Domena, UTM EP49 (leg. Černjavski, P. 13.08.1946., det. Černjavski, P., BEO 8932); Jabučji potok - greben, UTM EQ71, mešovita hrastova sastojina (leg. Černjavski, P. 29.07.1946., det. Černjavski, P., BEO 8935); Podarova Reka, desna padina ka seoskom putu, UTM EQ71, mešovita sastojina bukve i lipe (leg. Gajić, M. 11.07.1947., det. Gajić, M., BEO 8961); Miroč: Veliki Štrbac, Pecka bara - Ploča, UTM FQ03 (leg. Nikolić, V., Diklić, N. 25.09.1967., det. Diklić, N., BEO 43804); Sr Fruška gora: Iriški venac-Zmajevac, UTM DR00, Exp. N, bukova šuma (leg. Nikolić, V., Diklić, N., 28.05.1968., det. Diklić, N., BEO 43793); Š Kragujevac: Badnjevac, UTM DP98 (leg. Rudski, I., 30.04.1940., det. Rudski, I., BEO 8938); Beloševac, UTM DP96 (leg. Rudski, I. 21.05.1939., det. Rudski, I., BEO 8947); Drača, UTM DP87, Carpinetum (leg. Rudski, I. 06.06.1939., det. Rudski, I., BEO 8957); Grošnica, UTM DP96 (leg. Rudski, I. 24.05.1940., det. Rudski, I., BEO 8956); Mečkovac, UTM DP97 (leg. Rudski, I. 29.08.1939., det. Rudski, I., BEO 8946); Z Ovčarsko-kablarska klisura, UTM DP36 (leg. Nikolić, V., Diklić, N., 11.10.1955., det. Diklić, N., BEO 43788). 
60 Jovanović, M. ET AL.: Plants, FUnGI, AMPHIBIANS AND REPTILES FROM SERBIA

\section{NYMPHAEACEAE}

\section{Nymphaea alba L.}

Sr Obedska Bara: Obrež, UTM DQ15 (leg. Lindtner, V., 20.10.1946., det. Černjavski, P., BEO 6277, sub Castalia alba); 77 m (leg. Lindtner, V. 30.06.1946., det. Lindtner, V., BEO 40752, sub C. savensis).

\section{PAEONIACEAE}

\section{Paeonia daurica Andrews subsp. daurica}

K Kosovska Mitrovica: s. Kičica, UTM DN84 (leg. Matvejev, S., 28.05.1949., det. Nikolić, V., BEO 5409, sub P. corallina, rev. Hong, D.-J. 16.08.2003.); M Paštrik: Gorožup, UTM DM67, Exp. S, 500-1500 m, krečnjak, šuma Quercetum macedonicae i livade (leg. Nikolić, V., Diklić, N., Mladenović, S., 26.07.1979., det. Nikolić, V., BEO 45283, sub P. corallina, rev. Hong, D.-J. 16.08.2003.).

\section{Paeonia peregrina Mill.}

I Leskovik: vrh, UTM EP70, 1000 m, kamenjar (leg. Diklić, N., 22.06.1957., det. Diklić, N., BEO 45295, sub P. decora); UTM EP70 (leg. llić, Đ. 04.1909., det. Grebenščikov, O., BEO 5421, sub $P$. decora); Pirot: Gornje Krnjino, Petlovo bojište, UTM FN28 (leg. Vasić, O. 03.06.1990., det. Hong, D.-J., BEO 45294); Svrljig: Plužine, Visoke stene, UTM EP81, 480-600 m, krečnjak (leg. Lindtner, V. 10.05.1955., det. Lindtner, V., BEO 5430, sub P. decora); K Kosovska Mitrovica: s. Kičica, UTM DN84 (leg. Matvejev, S., 28.05.1949., det. Grebenščikov, O., BEO 5423, sub P. decora); Lipljane: Starogradsko, UTM EN00, 500 m, šuma sladuna i cera (leg. Diklić, N. 29.05.1971., det. Diklić, N., BEO 45296, sub P. decora); Priština: Gazimestan, kod spomenika, UTM DQ49 (leg. Nikolić, V. 25.05.1962., det. Nikolić, V., BEO 45305, sub P. decora); Sofalija, UTM EN12 (leg. Nikolić, V. 25.05.1962., det. Nikolić, V., BEO 45298, sub P. decora); SI Negotin: okolina, UTM FP29 (leg. Jovanović, A., 1908., det. Jovanović, A., BEO 5424, sub P. decora).

\section{PlumbaginaCEAE}

\section{Armeria rumelica Boiss.}

C Radan planina: Petrova gora, UTM EN46, 1400 m (leg. Nikolić, V., 23.07.1959., det. Nikolić, V., BEO 53393); Veliki pod, UTM EN36, Exp. S-W, livade (leg. Nikolić, V. 23.06.1960., det. Nikolić, V., BEO 53392); J Bujanovac: Binačka Morava, Lučane, UTM EM59 (leg. Nikolić, V., 28.06.1965., det. Nikolić, V., BEO 53387, sub A. vulgaris, rev. Buzurović, U. 25.02.2019.); Lopardinci, iznad sela, UTM EN60, kamenjari (leg. Diklić, N. 01.06.1963., det. Diklić, N., BEO 53383); Rujan: prevoj-Svinjište, pored puta, UTM EM68, $700 \mathrm{~m}$, silikat (leg. Tomović, G., Zlatković, B. 01.06.2014., det. Buzurović, U., BEO 53416); Vranje: Lebovac, UTM EN61 (leg. llić, Đ. 07.1910., det. Ilić, Đ., BEO 16657); JI Besna kobila, UTM FN00, planinske livade (leg. llić, Đ., 06., det. Ilić, Đ., BEO 16646); ispod vrha, UTM FN00, 1600-1900 m, livade i pašnjaci (leg. Nikolić, V., Diklić, N. 17.07.1968., det. Nikolić, V., Diklić, N., BEO 53395); kod planinarskog doma, UTM FN00, 15001600 m, livade (leg. Nikolić, V., Diklić, N. 22.07.1967., det. Nikolić, V., Diklić, N., BEO 53391); spomenik-vrh, UTM FN00, 1650-1922 m, pašnjaci i kamenjari (leg. Diklić, N., Mladenović, S. 15.07. 1979., det. Diklić, N., Mladenović, S., BEO 53390); Čemernik, UTM FN03 (leg. Černjavski, P. 19.08.1930., det. Černjavski, P., BEO 16641); Karamanica: pored puta, UTM FM08, 1528 m, silikat, visokoplaninske rudine (leg. Tomović, G., Tomović, LJ., Zlatković, B. 21.06.2014., det. Buzurović, U., BEO 53412); Krajište: Božica, periferija sela, UTM FN11, silikat, mezofilne livade i erodirane 
površine (leg. Niketić, M., Tomović, G. 29.06.2016., det. Niketić, M., BEO 53454); Ostrozub: Dobro Polje, UTM FN04, planinske livade (leg. Ilić, Đ. 06., det. Ilić, Đ., BEO 16644); Vardenik: Valožje, Brzaci (mahala), UTM FN01 (leg. Bokić, B., Radak, B. 17.06.2013., det. Buzurović, U., BEO 53435); Veliki Strešer, zapadna padina, UTM FN02, Exp. W, 1700 m, livade (leg. Nikolić, V., Diklić, N. 23.07.1967., det. Nikolić, V., Diklić, N., BEO 53389); Vlasina, UTM FN02 (leg. Ilić, Đ. 07.1910., det. Ilić, Đ., BEO 16631); ogolićeni breg i žbunje (leg. Černjavski, P. 09.08.1930., det. Černjavski, P., BEO 16640); Baltijance, UTM FN02 (leg. Ilić, Đ. 06.1910., det. Ilić, Đ., BEO 16656); Okruglica, UTM FN02 (leg. Bokić, B., Radak, B. 17.06.2013., det. Buzurović, U., BEO 53438); Klisura (s.), na putu za selo, UTM FN13 (leg. Diklić, N. 18.07.1958., det. Diklić, N., BEO 53382); Vranje: Crni vrh, UTM EN90, planinske livade (leg. Ilić, Đ. 06., det. Ilić, Đ., BEO 16645).

\section{Goniolimon tataricum (L.) Boiss. subsp. tataricum}

C Ibarska dolina: Raška, UTM DN69, serpentinit (leg. Lindtner, V., 23.06.1948., det. Lindtner, V., BEO 16583, sub G. collinum, rev. Buzurović, U. 04.03.2013.); Vlas, UTM DN69, serpentinit, kamenjari (leg. Pavlović, Z., Lindtner, V. 30.07.1955., det. Pavlović, Z., BEO 53464, sub G. collinum subsp. serbicum); Ušće, UTM DP61, serpentinit, stene (leg. Rajevski, L. 15.07.1950., det. Rajevski, L., BEO 16599, sub G. collinum subsp. serbicum); kod krsta na vrhu brda u centru mesta, UTM DP61, 348 m, ultramafiti (leg. Buzurović U., Đurović, S., Kabaš, E. 03.07.2016., det. Buzurović, U., BEO s.n.); Prokuplje: Hisar, UTM EN48 (leg. Jurišić, Ž. 03.06.1911., det. Jurišić, Ž., BEO 16611, sub Statice gmelini, rev. Buzurović, U. 04.04.2012.); (leg. Jurišić, Ž. 31.05.1911., det. Jurišić, Ž., BEO 16613, sub Statice gmelini, rev. Buzurović, U. 04.04.2012.); (leg. Nikolić, V. 05.07.1953., det. Nikolić, V., BEO 53256, sub G. serbicum); Sokolica, UTM EN48, dolomit (leg. Ružić, M. 07.1979., det. Ružić, M., BEO 53260, sub Statice gmelini, rev. Buzurović, U. 25.02.2015.); I Pirot - Bela Palanka, UTM FN28, krečnjak, pored puta (leg. Diklić, N., 15.06.1974., det. Diklić, N., BEO 53259, sub G. serbicum); K Ibarska dolina: Banjska, okolina, UTM DN85, serpentinit, kamenjari (leg. Pavlović, Z., 16.07. 1955., det. Pavlović, Z., BEO 53438); pored puta za Kosovsku Mitrovicu, UTM DN85 (leg. Nikolić, V., Diklić, N., Bogdanović, M. 12.07.1973., det. Diklić, N., BEO 53254, sub G. collinum, rev. Buzurović, U. 04.03.2013.); Sokolovina, UTM DN85, serpentinit, kamenjari (leg. Pavlović, Z. 17.07.1955., det. Pavlović, Z., BEO 53460); Kula, ispod vrha na sedlu, UTM DN85, travnjak (leg. Pavlović, Z. 23.06.1956., det. Pavlović, Z., BEO 53462); motel Simonida, UTM DN86, 496 m, ultramafiti (leg. Buzurović U., Đurović, S., Kabaš, E. 03.07.2016., det. Buzurović, U., BEO s.n.); Priština: Koznica, Lozište, UTM EN32 (leg. Jurišić, Ž. 27.06.1914., det. Jurišić, Ž., BEO 16600, sub G. collinum subsp. serbicum); M Peć: Klina, Pograđe, UTM DN72 (leg. Černjavski, P., 1933., det. Černjavski, P., BEO 16586, sub G. collinum, rev. Buzurović, U. 04.03.2013.).

\section{Limonium gmelinii (Willd.) Kuntze}

Bč Subotičko-horgoška peščara: Horgoš, UTM DS21, močvare i slatine (leg. Nikolić, V., Mladenović, S., 28.06.1981., det. Diklić, N., BEO 53280, sub Statice gmelini); Horgoš-Palić, UTM DS10, močvare i slatine (leg. Nikolić, V., Mladenović, S. 29.06.1981., det. Diklić, N., BEO 53287, sub Statice gmelini); Bt Kikinda: Melenci-Bašaid, pored puta, UTM DR54, livade, slatine (leg. Jovanović, M., Stojanović,A., 01.09.2011., det. Jovanović, M., BEO 53289); Novi Kneževac - Banatsko Aranđelovo, UTM DR39 (leg. Nikolić, V., Diklić, N. 02.06.1978., det. Diklić, N., BEO 53277, sub Statice gmelini); Vršac: Veliki Vršački rit, UTM ER10 (leg. Nojgebauer 08.1940., det. Nojgebauer, BEO 16612, sub Statice gmelini); Zrenjanin: Melenci, Banja Rusanda, UTM DR44 (leg. Tucakov, J. 09.1969., det. Diklić, N., BEO 53278, sub Statice gmelini); C Prokuplje: Oblačinsko jezero, UTM EN59 (leg. Jurišić, Ž., 04.06.1911., det. Jurišić, Ž., BEO 16605, sub Statice gmelini); (leg. Adamović, Ž. 19.08.1973., det. Diklić, N., BEO 53276, sub Statice gmelini); pored vode (leg. Diklić, N., Mladenović, S. 17.07.1985., det. Diklić, N., Mladenović, S., BEO 53272). 


\section{Plumbago europaea L.}

J Preševo: "Krnje brdo", vrh, UTM EM58, stene (leg. Diklić, N., 24.09.1961., det. Diklić, N., BEO 53252); Rujan: Cer (s.), UTM EM57, krečnjak, kamenjari (leg. Ranđelović, N. 03.08.1982., det. Ranđelović, N., BEO 53251).

\section{RANUNCULACEAE}

\section{Anemone sylvestris L.}

Bt Deliblatska peščara: Čardak, UTM EQ06 (leg. Grebenščikov, O., 06.05.1945., det. Grebenščikov, O., BEO 5751); Deliblato, UTM EQ06 (leg. Jurišić, J. 08.05.1922., det. Jurišić, J., BEO 5749); (leg. Miljković, B. 1929., det. Miljković, B., BEO 5756); dolina, UTM EQ07 (leg. Grebenščikov, O. 05.05.1948., det. Grebenščikov, O., BEO 5752); Korn bunar, UTM EQ07, in arenosis (leg. Soška, $T$. 15.05.1930., det. Soška, T., BEO 5748); Mramorak, UTM EQ06, In arenosis (leg. Grebenščikov, O. 17.05.1935., det. Grebenščikov, O., BEO 5754); Sr Fruška gora: Stražilovo, UTM DR10 (leg. Grebenščikov, O., 06.05.1949., det. Diklić, N., BEO 39848); (leg. Lindtner, V. 24.04.1959., det. Lindtner, V., BEO 39849).

\section{Hepatica nobilis Schreb.}

I Ozren: Lepterija, UTM EP73, Carpinetum orientalis (leg. Nikolić, V., Diklić, N., Bogdanović, M., 19.06.1972., det. Jovanović, M., BEO 39898); Sokobanja, Janior, UTM EP73, šibljak Carpinetum orientalis (leg. Diklić, N. 19.05.1957., det. Diklić, N., BEO 39890, sub Anemone hepatica); Lepterija iznad Moravice, UTM EP73, mladi bukovi šumarak (leg. Diklić, N. 21.05.1957., det. Diklić, N., BEO 39889, sub Anemone hepatica); Rtanj: iznad Lukova, UTM EP64, 900 m, In silvis (Fagetum) (leg. Grebenščikov, O. 07.04.1939., det. Grebenščikov, O., BEO 5668, sub Anemone hepatica); severna padina, UTM EP74, Exp. N, kamenjar (leg. Nikolić, V., Diklić, N., Bogdanović, M. 20.06.1972., det. Jovanović, M., BEO 39900); Sićevačka klisura: Sićevo, Radov dol, UTM EN99, in silvis (leg. Lindtner, V. 02.05.1954., det. Lindtner, V., BEO 5660, sub Anemone hepatica); Stara planina: Belan, Topli Do, UTM FN29, 1200 m, In fagetis (leg. Grebenščikov, O. 05.07.1946., det. Grebenščikov, O., BEO 5670, sub Anemone hepatica); Temska, iznad sela, UTM FN28, Exp. NW, QuercetoCarpinetum (leg. Nikolić, V. 26.03.1979., det. Nikolić, V., BEO 39886, sub Anemone hepatica); Temska - Topli Do, UTM FN28, Exp. SW, Quercetum (leg. Nikolić, V. 11.03.1979., det. Nikolić, V., BEO 39887, sub Anemone hepatica); Svrljiške planine, UTM FP00, Exp. N, bukova šuma (leg. Nikolić, V. 17.05.1957., det. Nikolić, V., BEO 39895, sub Anemone hepatica); Pleš, iznad sela Beloinje, UTM EP90, 780-820 m, bukova šuma (leg. Lindtner, V. 28.06.1955., det. Lindtner, V., BEO 5659, sub Anemone hepatica); Vidlič: Carev kladenac, UTM FN38, Exp. NW, 1000 m, krečnjak, Carpinetum (leg. Nikolić, V. 01.04.1979., det. Nikolić, V., BEO 39897, sub Anemone hepatica); J Leskovac: Bučje, Radujev krak, UTM EN76 (leg. Jurišić, Ž., 22.05.1905., det. Jurišić, Ž., BEO 5662, sub $H$. triloba); JZ Priboj, UTM CP82, serpentinit, in Pinetis (leg. Lindtner, V., 14.04.1949., det. Lindtner, V., BEO 5649, sub Anemone hepatica); Bič planina, UTM CP82, in Pinetis (leg. Lindtner, V. 14.04.1949., det. Lindtner, V., BEO 5648, sub Anemone hepatica); Prijepolje: Duboki pipik, UTM CP90, Exp. W, posečena bukova šuma (leg. Pavlović, Z. 15.04.1949., det. Pavlović, Z., BEO 5651, sub Anemone hepatica); Mileševka, UTM CP90, hrastova šuma (leg. Pavlović, Z. 14.04.1949., det. Krstić, T., BEO 5652, sub Anemone hepatica); bukova šuma (leg. Pavlović, Z. 14.04.1949., det. Pavlović, Z., BEO 5654, sub Anemone hepatica); Barje, UTM CP90, bukova šuma (leg. Pavlović, Z. 14.04.1949., det. Pavlović, Z., BEO 5653, sub Anemone hepatica); M Prokletije: Dečanska planina, UTM DN31, mešovita bukova šuma (leg. Černjavski, P., Rudski, l., Lindtner, V., 15.07.1933., det. Černjavski, P., Rudski, I., Lindtner, V., BEO 5664, sub Anemone hepatica); Mokra planina, Džora, Seunovo, UTM DN43, 1800 m (leg. Rudski, I. 28.06.1932., det. Rudski, I., BEO 5655, sub Anemone hepatica); Oklačka glava (Razvrš), UTM DN54 (leg. Rudski, I. 09.07.1932., det. Rudski, I., BEO 5656, sub Anemone hepatica); Nedžinat, UTM DN31, jelova šuma (leg. Černjavski, P., Rudski, I., Lindtner, V. 28.07.1933., det. 
Černjavski, P., Rudski, I., Lindtner, V., BEO 5666, sub Anemone hepatica); SI Đerdap: Donji Milanovac - Greben, drugi potok prema Grebenu, UTM EQ92, Exp. NW, 80-100 m, šuma grabića (leg. Nikolić, V., Diklić, N., Rakin, M., 13.09.1965., det. Diklić, N., BEO 39894, sub Anemone hepatica); Pesača, UTM EQ73, šuma (leg. Nikolić, V., Diklić, N. 22.04.1968., det. Nikolić, V., BEO 39893, sub Anemone hepatica); Tekija - Kovilovski potok, UTM FQ14 (leg. Nikolić, V., Diklić, N. 28.06.1967., det. Diklić, N., BEO 39885, sub Anemone hepatica); Majdanpek: Majdanpečka domena, UTM EQ71, mešovita bukovo-hrastova šuma (leg. Černjavski, P. 29.07.1946., det. Černjavski, P., BEO 5658, sub Anemone hepatica); Miroč: Krš, Golubinjska reka, UTM EQ92, Exp. S, Syringetum (leg. Diklić, N. 17.05.1955., det. Diklić, N., BEO 39892, sub Anemone hepatica); Veliki Štrbac, blizu vrha, UTM FQ03, Exp. W, 700 m, mešovita šuma (leg. Diklić, N. 20.05.1955., det. Diklić, N., BEO 39891, sub Anemone hepatica); Negotin: Luka, UTM FP29, šuma (leg. Jurišsić, ž. 12.03.1906., det. Jurišić, Ž., BEO 5661, sub Anemone hepatica); Z Studenica, UTM DP61, Studenica (leg. Rudski, I., 01.06.1940., det. Grebenščikov, O., BEO 5650, sub Anemone hepatica); Tara planina: Derventa, Drum Debo, UTM CP66 (leg. Jurišić, Ž. 19.06.1912., det. Jurišić, Ž., BEO 5663, sub $H$. triloba); Užice: okolina, UTM DP05, stene (leg. anonim. 03., det. anonim., BEO 5671, sub Anemone hepatica).

\section{Ranunculus aquatilis $\mathrm{L}$.}

Bt Kikinda, UTM DR57 (leg. Izmajlov, N., 08.05.1922., det. Izmajlov, N., BEO 6199); Padej, Betlehem, UTM DR37 (leg. Lindtner, V. 05.05.1955., det. Lindtner, V., BEO 6200); Novi Kneževac: Novi Kneževac - Banatski Aleksandrovac, UTM DR29 (leg. Nikolić, V., Diklić, N. 02.06.1978., det. Jovanović, M., BEO 40041); Sutjeska, UTM DR72, slatine (leg. Nikolić, V., Diklić, N. 03.06.1979., det. Jovanović, M., BEO 40039); JI Vlasina: Biljene bare, UTM FN13 (leg. Milovanović, D., 25.06.1947., det. Nikolić, V., BEO 6195); Vlasinska reka, ispod malog mosta, UTM FN13 (leg. Milovanović, D. 23.06.1947., det. Nikolić, V., BEO 6194); Vlasinsko jezero, UTM FN03, ploveće ostrvo sfagnuma (leg. Diklić, N. 17.07.1958., det. Nikolić, V., BEO 6196); pored jezera, UTM FN03 (leg. Nikolić, V., Diklić, N. 26.07.1972., det. Jovanović, M., BEO 40037).

\section{Ranunculus flabellifolius Heuff. ex Rchb.}

SI Đerdap: Pesača potok, UTM EQ73, stara bukova šuma. (leg. Diklić, N., 28.04.1977., det. Jovanović, M., BEO 40247); bukova šuma. (leg. Nikolić, V., Diklić, N. 22.04.1968., det. Jovanović, M., BEO 40257); mešovite i bukove šume. (leg. Nikolić, V., Diklić, N. 22.04.1976., det. Jovanović, M., BEO 40261).

\section{Ranunculus flammula $\mathrm{L}$. subsp. flammula}

JI Vlasina: Biljene bare, UTM FN13 (leg. Milovanović, D., 25.06.1947., det. Nikolić, V., BEO 5953); Vlasinsko jezero, UTM FN03, močvarne livade (leg. Nikolić, V., Diklić, N. 17.06.1967., det. Diklić, N., BEO 40275); brana, UTM FN03, močvara, sfagnum (leg. Sigunov, A. 18.09.1981., det. Jovanović, M., BEO 40279); JZ Pešter, UTM DN27, pašnjaci i livade (leg. Nikolić, V., Diklić, N., Mladenović, S., 19.07.1980., det. Jovanović, M., BEO 40278).

\section{Ranunculus lateriflorus DC.}

Bt Kikinda, UTM DR57 (leg. Slavnić, ž., 05.1941., det. Slavnić, Ž., BEO 5935); Novi Bečej, UTM DR34 (leg. Lindtner, V. 18.06.1955., det. Lindtner, V., BEO 5934); Žabalj, UTM DR22, slatine pored puta (leg. Nikolić, V., Diklić, N. 25.05.1973., det. Nikolić, V., BEO 40306).

\section{Ranunculus serbicus Vis.}

I Vidlič: Carev kladenac, planinarski dom, UTM FN38, 800-1000 m, krečnjak, čistine u šumi grabića (leg. Nikolić, V., Diklić, N., 16.06.1976., det. Jovanović, M., BEO 40678); Careva česma, 
planinarski dom, UTM FN38 (leg. Nikolić, V., Diklić, N. 21.06.1974., det. Nikolić, V., BEO 40687); J Vranje: Sobina, kraj potoka, UTM EN71, pokraj potoka (leg. llić, Đ., det. Ilić, Đ., BEO 5950); K Šar-planina: Brezovica, Brezovica - Stojkova kuća, UTM EM06, 900-1600 m, bukovo-jelova šuma (leg. Nikolić, V., Diklić, N., Mladenović, S., 24.07.1976., det. Nikolić, V., BEO 40686); pojas bukove šume (leg. Nikolić, V., Diklić, N., Bogdanović, M. 22.07.1974., det. Nikolić, V., BEO 40689); Suva reka, prema Jažinačkom jezeru, UTM DM96, 1400-1900 m, mešovita šuma. (leg. Nikolić, V., Diklić, N., Mladenović, S. 20.07.1978., det. Nikolić, V., BEO 40683); M Prokletije: Mokra planina, Oklačka glava (Razvrš), UTM DN54 (leg. Rudski, I., 09.07.1932., det. Diklić, N., BEO 40680); Z Tara planina: Kaluđerske bare, UTM CP86 (leg. Nikolić, V., Diklić, N., Mladenović, S., 20.06.1978., det. Jovanović, M., BEO 40679).

\section{RHAMNACEAE}

\section{Frangula rupestris (Scop.) Schur}

I Sićevačka klisura: Ostrvica, Man. Sv. Petka, UTM EN99, zajednica žalfije (leg. Nikolić, V., Diklić, N., Bogdanović, M., 19.06.1973., det. Jovanović, M., BEO 44854); kamenjar sa Salvia officinalis (leg. Nikolić, V., Diklić, N., Rakin, M. 26.07.1964., det. Diklić, N., BEO 44857); Tupižnica: zapadna padina, UTM EP94, Exp. W, šibljak (leg. Diklić, N. 23.07.1962., det. Diklić, N., BEO 44864, sub Rhamnus rupestris); JZ Priboj: Oštrik, UTM CP91, serpentinit, kamenjari (leg. Nikolić, V., Diklić, N., Mladenović, S., 22.06.1978., det. Jovanović, M., BEO 44853); M Prokletije: Nedžinat, severne padine prema Bistrici, UTM DN31, Exp. N, 1200-1400 m (leg. Nikolić, V., Diklić, N., 25.07.1971., det. Nikolić, V., BEO 44860, sub Rhamnus rupestris); SI Kučaj: Malinik, UTM EP76, stene (leg. Diklić, N., 13.06.1965., det. Nikolić, V., BEO 44859, sub Rhamnus rupestris); Stol (Bor), UTM EP99, Exp. S, 800-1100 m, krečnjak, pašnjaci i kamenjari (leg. Nikolić, V., Diklić, N. 12.07.1985., det. Šilić, Č., BEO 44851); UTM EP88, Exp. SW, Stipa grafiana (leg. Nikolić, V., Diklić, N. 29.06.1962., det. Jovanović, M., BEO 44855); Veliki krš: Kovasla, UTM EP89, kamenjar (leg. Diklić, N. 16.07.1959., det. Diklić, N., BEO 44892, sub Rhamnus rupestris); Z Tara planina: Kaluđerske bare, Vidikovac, UTM CP86, 800 m, stene (leg. Diklić, N., 01.07.1965., det. Diklić, N., BEO 44858, sub Rhamnus rupestris); kanjon Dervente, UTM CP66 (leg. Nikolić, V., Diklić, N., Rakin, M. 14.07.1963., det. Diklić, N., BEO 44863, sub Rhamnus rupestris); Rača, UTM CP86, šuma crnog bora (leg. Diklić, N., Rakin, M. 16.07.1963., det. Nikolić, V., BEO 44861).

\section{ROSACEAE}

\section{Potentilla apennina Ten. subsp. apennina}

I Suva planina: Sokolov kamen, UTM EN98, $1552 \mathrm{~m}$, kamenjari (leg. Nikolić, V., Diklić, N., Bogdanović, M., 17.07.1974., det. Diklić, N., BEO 46331); Trem, UTM EN98, $1500 \mathrm{~m}$, kamenjari (leg. Nikolić, V., Diklić, N., Mladenović, S. 26.07.1977., det. Diklić, N., BEO 46326); vrh, UTM EQ83, stene (leg. Nikolić, V., Diklić, N., Bogdanović, M. 18.06.1973., det. Diklić, N., BEO 46327); $\underline{\text { M }}$ Prokletije: Koprivnik, UTM DN31, 2200 m, in rupibus (leg. Grebenščikov, O., 09.07.1939., det. Grebenščikov, O., BEO 11035); Nedžinat, UTM DN31, 2200-2450 m, osuline i kamenjari (leg. Nikolić, V., Diklić, N., Bogdanović, M. 15.07.1973., det. Diklić, N., BEO 46328); Nedžinatsko jezero, UTM DN31, Exp. N, 2000 m, krečnjak, mešovita četinarska šuma (leg. Nikolić, V., Diklić, N. 27.07.1971., det. Diklić, N., BEO 46329).

\section{Spiraea cana Waldst. \& Kit.}

JZ Novi Pazar: Gradina, Bačvar (brdo), Ras (pašnjak), UTM DN57, pašnjak (leg. Nikolić, V., Diklić, N., 16.05.1985., det. Jovanović, M., BEO 46019); Z Drina: Derventa-Grlac, UTM CP67 (leg. 
Jurišić, Ž., 19.06.1912., det. Jurišić, Ž., BEO 10824); Rzav: Val ispod Tustog brda, carinarnica mokrogorska, UTM CP92 (leg. Jurišić, Ž. 22.06.1912., det. Jurišić, Ž., BEO 10823).

\section{RUTACEAE}

\section{Haplophyllum suaveolens (DC.) G. Don}

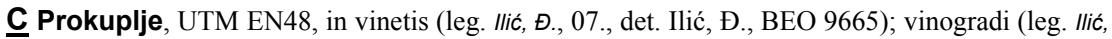
๑. 06.1909., det. Wraber, T., BEO 9676); J Aleksinac: Bobovište, UTM EP91, neobrađena mesta (leg. llić, Đ., 04., det. Ilić, Đ., BEO 9664); (leg. lilić, Đ., det. Wraber, T., BEO 9675).

\section{Ruta graveolens $\mathrm{L}$.}

I Sićevačka klisura: Ostrvica, Man. Sv. Petka, UTM EN99, zajednica žalfije i pelina (leg. Nikolić, V., Diklić, N., 27.05.1966., det. Nikolić, V., BEO 44287); goleti (leg. Diklić, N. 10.06.1971., det. Nikolić, V., BEO 44288); zajednica žalfije (leg. Nikolić, V., Diklić, N., Bogdanović, M. 19.06.1973., det. Nikolić, V., BEO 44291); Carpinetum orientalis (leg. Diklić, N. 26.06.1963., det. Diklić, N., BEO 44293); Sićevo, UTM EN89 (leg. ilić, Đ., det. ilić, Đ., BEO 9616); (leg. Jurišíc, Ž., det. Jurišić, Ž., BEO 9617); M Paštrik: Gorožup, UTM DM67, Exp. S, 500-1500 m, krečnjak, šuma Quercetum macedonicae i livade (leg. Nikolić, V., Diklić, N., Mladenović, S., 25.07.1979., det. Diklić, N., BEO 44294); SI Đerdap: Sip-Tekija, UTM FQ24, šuma grabića (Carpinus orientalis) (leg. Diklić, N., 29.07.1964., det. Diklić, N., BEO 44290).

\section{SAXIFRAGACEAE}

\section{Saxifraga bulbifera L.}

C Pasjača, UTM EN57, Exp. SW, hrastova šuma (leg. Nikolić, V., 13.05.1959., det. Jovanović, M., BEO 45826); Vidojevica, UTM EN47, livada (leg. Nikolić, V. 15.05.1959., det. Jovanović, M., BEO 45825); I Niš, UTM EN79 (leg. Jurišić, Ž., 05.1911., det. Jurišić, Ž., BEO 10587); J Binačka Morava: Končulj, UTM EN50 (leg. Jurišić, Ž., 10.05.1914., det. Jurišić, Ž., BEO 10588); Lučane, okolina, UTM EM59 (leg. Nikolić, V. 03.06.1967., det. Jovanović, M., BEO 45827); Vranje: Vrtogoš, Sv. Ilija, UTM EN60, 1200 m, livade (leg. Diklić, N. 02.06.1980., det. Diklić, N., BEO 45829); JI klisura Pčinje: Man. Prohor Pčinjski, UTM EM78 (leg. Nikolić, V., Diklić, N., 15.05.1975., det. Diklić, N., BEO 45831); Pčinja: Šajince-Trgovište, UTM EM89, kamenjar (leg. Nikolić, V., Diklić, N. 09.05. 1973., det. Diklić, N., BEO 45830); JZ Novi Pazar: Đurđevi Stupovi, UTM DN57, Exp. NE, 35$40^{\circ}$ (leg. Nikolić, V., Diklić, N., 17.05.1985., det. Jovanović, M., BEO 45824); Raičinovića banja, pored potoka, UTM DN47, pored potoka (leg. Nikolić, V., Diklić, N. 14.05.1985., det. Jovanović, M., BEO 45823); K K Gornje Nerodimlje, UTM EM09 (leg. Jurišsić, ž., 03.05.1914., det. Jurišić, Ž., BEO 10589).

\section{Saxifraga glabella Bertol.}

K Šar-planina: Kobilica, UTM DM96, c. 2400 m, pašnjaci i kamenjari (leg. Nikolić, V., Diklić, N., Mladenović, S., 19.07.1977., det. Diklić, N., BEO 45844); 1800-2300 m, pašnjaci i kamenjari (leg. Nikolić, V., Diklić, N., Mladenović, S. 18.07.1978., det. Diklić, N., BEO 45846); Konjuška, padine prema Bistri, UTM DM96, 2000-2400 m (leg. Nikolić, V., Diklić, N., Mladenović, S. 24.07.1978., det. Diklić, N., BEO 45841); Prevalac-Bistra, UTM DM96, 1600-2200 m, kamenjari (leg. Nikolić, V., Diklić, N. 23.07.1975., det. Diklić, N., BEO 45845); $\underline{M}$ Koritnik: Prizren, UTM DM66 (leg. Oehm, H., 09.1937., det. Soška, T., BEO 10680); Prokletije: Koprivnik, UTM DN31, $2200 \mathrm{~m}$, in rupibus (leg. Grebenščikov, $O$. 10.07.1939., det. Grebenščikov, O., BEO 10677); Nedžinat, UTM DN31, 2200-2450 m, osuline i 
kamenjari (leg. Nikolić, V., Diklić, N., Bogdanović, M. 15.07.1973., det. Diklić, N., BEO 45840); Pločica, pored Bistrice kod Pločanskih stanova, UTM DN21 (leg. Černjavski, P., Rudski, I., Lindtner, V. 18.07.1933., det. Černjavski, P., BEO 10632, sub S. adscendens, rev. Niketić, M. 08.1994.).

\section{ULMACEAE}

\section{Celtis australis L. subsp. australis}

Bt Kikinda, UTM DR57, u bašti (leg. Izmajlov, N., 04.04.1925., det. Izmajlov, N., BEO 2240); SI Đerdap: Boljetinska reka, prema Dobri, UTM EQ83, šuma Celtis australis-Juglans regia (leg. Diklić, N., 03.08.1966., det. Nikolić, V., BEO 36674); ušće pored Dunava, UTM EQ83 (leg. Diklić, N. 22.10.1965., det. Nikolić, V., BEO 36672); Miroč: Veliki Štrbac, Pecka bara - lugarnica, UTM FQ03 (leg. Nikolić, V., Diklić, N. 19.05.1967., det. Nikolić, V., BEO 36677); Pecka bara - Ploča, UTM FQ03 (leg. Nikolić, V., Diklić, N. 25.04.1967., det. Nikolić, V., BEO 36673).

\section{URTICACEAE}

\section{Parietaria serbica Pančić}

I Bela Palanka: Bela Palanka-Pirot, UTM FN08, krečnjak, stene iznad puta (leg. Nikolić, V., Diklić, N., 15.06.1974., det. Nikolić, V., BEO 36719); Bor: Rgotski kamen, UTM EP97, kamenjar (leg. Nikolić, V., Diklić, N., Bogdanović, M. 15.06.1973., det. Nikolić, V., BEO 36720); Jelašnička klisura, UTM EN89, krečnjak, kamenjari (leg. Diklić, N., Mladenović, S. 14.07.1979., det. Nikolić, V., BEO 36711); stene (leg. Nikolić, V., Diklić, N. 16.06.1967., det. Nikolić, V., BEO 36713); stene (leg. Nikolić, V., Diklić, N. 24.05.1964., det. Diklić, N., BEO 36717); stene (leg. Nikolić, V. 09.04.1975., det. Nikolić, V., BEO 36722); Sićevačka klisura: Sićevo, UTM EN89 (leg. llić, Đ., det. Ilić, Đ., BEO 2307); SI Gornjačka klisura: Man. Gornjak, UTM EQ40, stene (leg. Nikolić, V., Diklić, N., Bogdanović, M., 21.06.1972., det. Nikolić, V., BEO 36718).

\section{Urtica kioviensis Rogow.}

Sr Obedska Bara, UTM DQ25 (leg. Milovanović, D., 11.06.1947., det. Černjavski, P., BEO 36690); Obrež, UTM DQ15 (leg. Černjavski, P. 26.06.1948., det. Lintner, V., BEO 36688); (leg. Černjavski, P. 22.09.1946., det. Lintner, V., BEO 36689).

\section{FUNGI Pezizomycetes MORCHELLACEAE}

\section{Morchella elata Fr.}

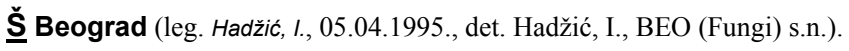

\section{Morchella esculenta (L.) Pers.}

Sr Sremska Mitrovica (leg. Hadžić, I., 24.04.1996., det. Hadžić, I., BEO (Fungi) s.n., sub M.

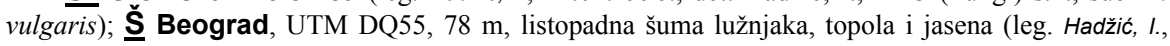
02.04.1992., det. Hadžić, I., BEO (Fungi) s.n.); UTM DQ65, 225 m, listopadna šuma (leg. Hadžić, I. 03.04.1995., det. Hadžić, I., BEO (Fungi) s.n.). 


\section{TUBERACEAE}

\section{Tuber aestivum (Wulfen) Spreng.}

Bt Bela Crkva: Jasenovo, UTM EQ27, 76 m, šume lužnjaka, topola i jasena u dolini reke (Ulmenion minoris) (leg. Milenković, M. et al., 10.10.1995., det. Milenković, M., BEO (Fungi) s.n.); Straža, UTM EQ27, $75 \mathrm{~m}$, šume lužnjaka, topola i jasena u dolini reke (Ulmenion minoris), hrast lužnjak (leg. Milenković, M. et al. 10.10.1995., det. Milenković, M., BEO (Fungi) s.n.); BEO (Fungi) s.n.); BEO (Fungi) s.n.); Deliblatska peščara, UTM EQ17, $150 \mathrm{~m}$, hrastove brdske šume, Querceto-

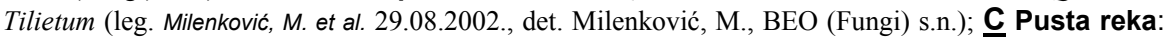
Dubovo, UTM EN57, $300 \mathrm{~m}$, hrastove brdske šume, sastojine hrasta cera (leg. Milenković, M. et al., 19.08.2005., det. Milenković, M., BEO (Fungi) s.n.); I Aleksinac, UTM EP52, 170 m, šume lužnjaka, topola i jasena u dolini reke (Ulmenion minoris) (leg. Milenković, M. et al., 18.11.1998., det. Milenković, M., BEO (Fungi) s.n.); Svrljig, UTM EP71, $460 \mathrm{~m}$, hrastove brdske šume (leg. Milenković, M. et al. 23.10.1992., det. Milenković, M., BEO (Fungi) s.n.); Zaplanje: Krastavče, UTM EN88, 450 m, hrastove brdske šume (leg. Milenković, M. et al. 20.08.2005., det. Milenković, M., BEO (Fungi) s.n.); Po Požarevac: Bratinac, UTM EQ14, $80 \mathrm{~m}$, šume lužnjaka, topola i jasena u dolini reke (Ulmenion minoris) (leg. Milenković, M. et al., 17.12.1992., det. Milenković, M., BEO (Fungi) s.n.); Resava: Roanda, UTM EP29, $200 \mathrm{~m}$, šume lužnjaka, topola i jasena u dolini reke (Ulmenion minoris), topola (leg. Milenković, M. et al. 19.11.1998., det. Milenković, M., BEO (Fungi) s.n.); Smederevska Palanka, UTM DQ91, 100 m, šume lužnjaka, topola i jasena u dolini reke (Ulmenion minoris), topola (leg. Milenković, M. et al. 11.11.1995., det. Milenković, M., BEO (Fungi) s.n.); Stig: Požarevac, UTM EQ14, 80 m, šume lužnjaka, topola i jasena u dolini reke (Ulmenion minoris) (leg. Milenković, M. et al. 03.11.1998., det. Milenković, M., BEO (Fungi) s.n.); UTM EQ22, 95 m, šume lužnjaka, topola i jasena u dolini reke (Ulmenion minoris), leska (leg. Milenković, M. et al. 24.10.1992., det. Milenković, M., BEO (Fungi) s.n.); Velika Plana, UTM EQ01, 70 m, šume lužnjaka, topola i jasena u dolini reke (Ulmenion minoris), hrast lužnjak (leg. Milenković, M. et al. 18.11.1998., det. Milenković, M., BEO (Fungi) s.n.); $\underline{\text { Sr }}$ Fruška gora, $480 \mathrm{~m}$, hrastove brdske šume, Querco carpinetum betuli (leg. Milenković, M. et al., 24.11.1995., det. Milenković, M., BEO (Fungi) s.n.); 500 m, hrastove brdske šume (leg. Milenković, M. et al. 24.11.1995., det. Milenković, M., BEO (Fungi) s.n.); Zemun: Dobanovci, UTM DQ46, 85 m, listopadna šuma (leg. Milenković, M. et al. 03.03.1997., det. Milenković, M., BEO (Fungi) s.n.); BEO (Fungi) s.n.); Surčinsko polje, UTM DQ46, $85 \mathrm{~m}$, listopadna šuma (leg. Milenković, M. et al. 24.11.1995., det. Milenković, M., BEO (Fungi) s.n.);

SZ Lajkovac: Slovac, UTM DQ20, 120 m, hrastove brdske šume, Querceto-Tilietum (leg. Milenković, M. et al., 03.08.2006., det. Milenković, M., BEO (Fungi) s.n.); Šabac, UTM CQ95, $70 \mathrm{~m}$, šume lužnjaka, topola i jasena u dolini reke (Ulmenion minoris), hrast lužnjak (leg. Milenković, M. et al. 07.09.1996., det. Milenković, M., BEO (Fungi) s.n.); 75 m, šume lužnjaka, topola i jasena u dolini reke (Ulmenion minoris) (leg. Milenković, M. et al. 06.11.1999., det. Milenković, M., BEO (Fungi) s.n.); Valjevo, UTM DP09, $260 \mathrm{~m}$, hrastove brdske šume, sastojine hrasta cera (leg. Milenković, M. et al. 01.08.2006., det. Milenković, M., BEO (Fungi) s.n.); S̆ Beograd: Košutnjak, UTM DQ55, $171 \mathrm{~m}$, hrastove brdske šume (leg. Milenković, M. et al., 08.08.1997., det. Milenković, M., BEO (Fungi) s.n.); Mala Moštanica, UTM DQ44, $150 \mathrm{~m}$, hrastove brdske šume, sastojine hrasta cera (leg. Milenković, $M$. et al. 28.06.1997., det. Milenković, M., BEO (Fungi) s.n.); UTM DQ55, $70 \mathrm{~m}$, parkovska šuma (leg. Milenković, M. et al. 05.09.1992., det. Milenković, M., BEO (Fungi) s.n.); 75 m, šume lužnjaka, topola i jasena u dolini reke (Ulmenion minoris) (leg. Milenković, M. et al. 15.10.1996., det. Milenković, M., BEO (Fungi) s.n.); (leg. Milenković, M. et al. 15.05.2015., det. Milenković, M., BEO (Fungi) s.n.); $70 \mathrm{~m}$, parkovska šuma, leska (leg. Milenković, M. et al. 15.09.1994., det. Milenković, M., BEO (Fungi) s.n.); Kolubara: Lazarevac, UTM DQ31, 110 m, šume lužnjaka, topola i jasena u dolini reke (Ulmenion minoris), hrast lužnjak (leg. Milenković, M. et al. 05.11.2005., det. Milenković, M., BEO (Fungi) s.n.); Kosmaj, UTM DQ62, $515 \mathrm{~m}$, hrastove brdske šume, Querceto-Tilietum (leg. Milenković, M. et al. 02.02.2002., det. Milenković, M., BEO (Fungi) s.n.); $565 \mathrm{~m}$, hrastove brdske šume (leg. Milenković, M. et al. 27.10.2002., det. Milenković, M., BEO (Fungi) s.n.); Mladenovac: Amerić, UTM DQ72, $200 \mathrm{~m}$, hrastove brdske šume (leg. Milenković, M. et al. 29.01.2002., det. Milenković, M., BEO (Fungi) s.n.); UTM DQ71, 80 m, šume lužnjaka, topola i jasena u dolini reke (Ulmenion minoris) (leg. Milenković, M. et 
al. 13.01.2002., det. Milenković, M., BEO (Fungi) s.n.); Obrenovac: Mala Moštanica, UTM DQ44, $100 \mathrm{~m}$, hrastove brdske šume (leg. Milenković, M. et al. 04.09.1996., det. Milenković, M., BEO (Fungi) s.n.); $150 \mathrm{~m}$, hrastove brdske šume, sastojine hrasta cera (leg. Milenković, M. et al. 01.09.2001., det. Milenković, M., BEO (Fungi) s.n.); 12.04.2003., det. Milenković, M., BEO (Fungi) s.n.); 13.05.2001., det. Milenković, M., BEO (Fungi) s.n.); 20.10.2000., det. Milenković, M., BEO (Fungi) s.n.); 28.10.2001., det. Milenković, M., BEO (Fungi) s.n.); Umka, UTM DQ44, 160 m, hrastove brdske šume, sastojine hrasta cera (leg. Milenković, M. et al. 25.06.1997., det. Milenković, M., BEO (Fungi) s.n.); UTM DQ34, 75 m, šume lužnjaka, topola i jasena u dolini reke (Ulmenion minoris) (leg. Milenković, M. et al. 05.10.1996., det. Milenković, M., BEO (Fungi) s.n.); Smederevska Palanka: Glibovac, UTM DQ91, 100 m, šume lužnjaka, topola i jasena u dolini reke (Ulmenion minoris) (leg. Milenković, M. et al. 01.12.2001., det. Milenković, M., BEO (Fungi) s.n.); 02.12.1994., det. Milenković, M., BEO (Fungi) s.n.); 24.10.1995., det. Milenković, M., BEO (Fungi) s.n.); šume lužnjaka, topola i jasena u dolini reke (Ulmenion minoris), hrast lužnjak (leg. Milenković, M. et al. 06.10.1995., det. Milenković, M., BEO (Fungi) s.n.); Z Tara planina, UTM CP76, $1060 \mathrm{~m}$, mešovita lišćarsko-četinarska šuma, Fagetum (leg. Milenković, M. et al., 29.09.2004., det. Milenković, M., BEO (Fungi) s.n.).

\section{Tuber macrosporum Vittad.}

Bt Banatska Palanka, UTM EQ26, 75 m, listopadna šuma (leg. Milenković, M. et al., 11.10. 1995., det. Milenković, M., BEO (Fungi) s.n.); Bela Crkva: Jasenovo, UTM EQ27, 76 m, šume lužnjaka, topola i jasena u dolini reke (Ulmenion minoris) (leg. Milenković, M. et al. 10.10.1995., det. Milenković, M., BEO (Fungi) s.n.); Straža, UTM EQ27, 75 m, šume lužnjaka, topola i jasena u dolini reke (Ulmenion minoris), hrast lužnjak (leg. Milenković, M. et al. 10.10.1995., det. Milenković, M., BEO (Fungi) s.n.); Pančevo, UTM DQ76, $70 \mathrm{~m}$, šume lužnjaka, topola i jasena u dolini reke (Ulmenion minoris), hrast lužnjak (leg. Milenković, M. et al. 02.11.1994., det. Milenković, M., BEO (Fungi) s.n.); 03.03.1995., det. Milenković, M., BEO (Fungi) s.n.); 18.10.1995., det. Milenković, M., BEO (Fungi) s.n.); BEO (Fungi) s.n.); 19.10.1994., det. Milenković, M., BEO (Fungi) s.n.); 20.12.1994., det. Milenković, M., BEO (Fungi) s.n.); 23.11.1994., det. Milenković, M., BEO (Fungi) s.n.); 29.10.1994., det. Milenković, M., BEO (Fungi) s.n.); BEO (Fungi) s.n.); Uzdin, UTM DR60, 75 m, šume lužnjaka, topola i jasena u dolini reke (Ulmenion minoris), topola (leg. Milenković, M. et al. 08.10.1995., det. Milenković, M., BEO (Fungi) s.n.); $\underline{\mathbf{C}}$ Trstenik, UTM DP92, $170 \mathrm{~m}$, šume lužnjaka, topola i jasena u dolini reke (Ulmenion minoris), hrast lužnjak (leg. Milenković, M. et al., 20.11.2005., det. Milenković, M., BEO (Fungi) s.n.); ! Aleksinac, UTM EP52, $170 \mathrm{~m}$, šume lužnjaka, topola i jasena u dolini reke (Ulmenion minoris) (leg. Milenković, M. et al., 18.11.1998., det. Milenković, M., BEO (Fungi) s.n.); Istočna Srbija (leg. Milenković, M. et al. 22.10.1992., det. Milenković, M., BEO (Fungi) s.n.); BEO (Fungi) s.n.); Svrljig: Lozan, UTM FP00, $450 \mathrm{~m}$, hrastove brdske šume (leg. Milenković, M. et al. 22.10.1992., det. Milenković, M., BEO (Fungi) s.n.); UTM EP80, $550 \mathrm{~m}$, listopadna šuma (leg. Milenković, M. et al. 21.10.1992., det. Milenković, M., BEO (Fungi) s.n.); Po Batočina, UTM EP08, $105 \mathrm{~m}$, šume lužnjaka, topola i jasena u dolini reke (Ulmenion minoris), topola (leg. Milenković, M. et al., 13.10.1994., det. Milenković, M., BEO (Fungi) s.n.); BEO (Fungi) s.n.); Lapovo: Markovac, UTM EP19, $100 \mathrm{~m}$, šume lužnjaka, topola i jasena u dolini reke (Ulmenion minoris) (leg. Milenković, M. et al. 21.11.2005., det. Milenković, M., BEO (Fungi) s.n.); Mlava: Petrovac, Orljevo, UTM EQ22, 100 m, šume lužnjaka, topola i jasena u dolini reke (Ulmenion minoris) (leg. Milenković, M. et al. 13.10.1994., det. Milenković, M., BEO (Fungi) s.n.); Resava: Roanda, UTM EP29, 200 m, šume lužnjaka, topola i jasena u dolini reke (Ulmenion minoris), topola (leg. Milenković, M. et al. 19.11.1998., det. Milenković, M., BEO (Fungi) s.n.); Smederevska Palanka: Glibovac, UTM DQ91, 100 m, šume lužnjaka, topola i jasena u dolini reke (Ulmenion minoris) (leg. Milenković, M. et al. 02.12.1994., det. Milenković, M., BEO (Fungi) s.n.); 24.10.1995., det. Milenković, M., BEO (Fungi) s.n.); UTM DQ91, 100 m, šume lužnjaka, topola i jasena u dolini reke (Ulmenion minoris), topola (leg. Milenković, M. et al. 11.11.1995., det. Milenković, M., BEO (Fungi) s.n.); Stig: Požarevac, UTM EQ13, 80 m, šume lužnjaka, topola i jasena u dolini reke (Ulmenion minoris), hrast lužnjak (leg. Milenković, M. et al. 03.11.1998., det. Milenković, M., BEO (Fungi) s.n.); UTM EQ14, 80 m, šume lužnjaka, topola i jasena u dolini reke (Ulmenion minoris) (leg. Milenković, M. et al. 03.11.1998., det. Milenković, M., BEO (Fungi) s.n.); SZ Mačva: Bogatić, UTM CQ86, 80 m, šume lužnjaka, topola i jasena u dolini reke (Ulmenion 
minoris) (leg. Milenković, M. et al., 10.11.1998., det. Milenković, M., BEO (Fungi) s.n.); Povlen: Stubica, UTM EQ14, $80 \mathrm{~m}$, šume lužnjaka, topola i jasena u dolini reke (Ulmenion minoris) (leg. Milenković, M. et al. 14.10.1994., det. Milenković, M., BEO (Fungi) s.n.); Valjevo, UTM DP09, 260 m, hrastove brdske šume, sastojine hrasta cera (leg. Milenković, M. et al. 15.02.2008., det. Milenković, M., BEO (Fungi) s.n.); UTM DQ10, 160 m, šume lužnjaka, topola i jasena u dolini reke (Ulmenion minoris) (leg. Milenković, M. et al. 06.11.2005., det. Milenković, M., BEO (Fungi) s.n.); Š Barajevo, UTM DQ53, 130 m, šume lužnjaka, topola i jasena u dolini reke (Ulmenion minoris) (leg. Milenković, M. et al., 04.11.1998., det. Milenković, M., BEO (Fungi) s.n.); Jasenica: Saranovo, UTM DP89, 130 m, šume lužnjaka, topola i jasena u dolini reke (Ulmenion minoris), topola (leg. Milenković, M. et al. 01.12.2001., det. Milenković, M., BEO (Fungi) s.n.); 04.11.1995., det. Milenković, M., BEO (Fungi) s.n.); 14.12.1994., det. Milenković, M., BEO (Fungi) s.n.); 15.12.1995., det. Milenković, M., BEO (Fungi) s.n.); 17.10.1998., det. Milenković, M., BEO (Fungi) s.n.); 18.11.1994., det. Milenković, M., BEO (Fungi) s.n.); Kolubara: Lazarevac, Kolubarski kopovi uglja, UTM DQ32, 105 m, šume lužnjaka, topola i jasena u dolini reke (Ulmenion minoris) (leg. Milenković, M. et al. 06.12.2001., det. Milenković, M., BEO (Fungi) s.n.); UTM DQ42, 150 m, šume lužnjaka, topola i jasena u dolini reke (Ulmenion minoris) (leg. Milenković, M. et al. 04.11.1998., det. Milenković, M., BEO (Fungi) s.n.); Sokolovo, UTM DQ42, 90 m, šume lužnjaka, topola i jasena u dolini reke (Ulmenion minoris) (leg. Milenković, M. et al. 04.11.1998., det. Milenković, M., BEO (Fungi) s.n.); Veliki Crljeni, UTM DQ42, $100 \mathrm{~m}$, šume lužnjaka, topola i jasena u dolini reke (Ulmenion minoris) (leg. Milenković, M. et al. 04.11.1998., det. Milenković, M., BEO (Fungi) s.n.); Mladenovac: Amerić, UTM DQ72, 180 m, hrastove brdske šume (leg. Milenković, M. et al. 13.01.2002., det. Milenković, M., BEO (Fungi) s.n.); UTM DQ71, 130 m, šume lužnjaka, topola i jasena u dolini reke (Ulmenion minoris) (leg. Milenković, M. et al. 01.12.1995., det. Milenković, M., BEO (Fungi) s.n.); Obrenovac: Mala Moštanica, UTM DQ44, $125 \mathrm{~m}$, hrastove brdske šume, sastojine hrasta cera (leg. Milenković, M. et al. 30.11.1992., det. Milenković, M., BEO (Fungi) s.n.); 150 m, hrastove brdske šume, sastojine hrasta cera (leg. Milenković, M. et al. 05.10.1996., det. Milenković, M., BEO (Fungi) s.n.); Umka, UTM DQ44, 145 m, parkovska šuma, breza (leg. Milenković, M. et al. 06.11.2005., det. Milenković, M., BEO (Fungi) s.n.); 26.11.2005., det. Milenković, M., BEO (Fungi) s.n.); UTM DQ35, 75 m, šume lužnjaka, topola i jasena u dolini reke (Ulmenion minoris), hrast lužnjak (leg. Milenković, M. et al. 05.11.1994., det. Milenković, M., BEO (Fungi) s.n.); Smederevo: Ralja, UTM DQ93, 80 m, šume lužnjaka, topola i jasena u dolini reke (Ulmenion minoris) (leg. Milenković, M. et al. 30.11.1996., det. Milenković, M., BEO (Fungi) s.n.); 100 m, šume lužnjaka, topola i jasena u dolini reke (Ulmenion minoris) (leg. Milenković, M. et al. 17.10.1998., det. Milenković, M., BEO (Fungi) s.n.); Smederevska Palanka: Glibovac, UTM DQ91, 100 m, šume lužnjaka, topola i jasena u dolini reke (Ulmenion minoris), hrast lužnjak (leg. Milenković, M. et al. 06.10.1995., det. Milenković, M., BEO (Fungi) s.n.); Stepojevac: Veliki Borak, UTM DQ53, 150 m, listopadna šuma (leg. Milenković, M. et al. 14.11.1995., det. Milenković, M., BEO (Fungi) s.n.).

\section{Tuber magnatum Picco}

Bt Pančevo: Crepaja, UTM DQ78, $80 \mathrm{~m}$, listopadna šuma, leska (leg. Milenković, M. et al., 08.11.2014., det. Milenković, M., BEO (Fungi) s.n.); UTM DQ76, 70 m, šume lužnjaka, topola i jasena u dolini reke (Ulmenion minoris), topola (leg. Milenković, M. et al. 20.10.1994., det. Milenković, M., BEO (Fungi) s.n.); PO Batočina, UTM EP08, $120 \mathrm{~m}$, šume lužnjaka, topola i jasena u dolini reke (Ulmenion minoris), hrast lužnjak (leg. Milenković, M. et al., 19.10.1992., det. Milenković, M., BEO (Fungi) s.n.); Po dolina Velike Morave, $80 \mathrm{~m}$, šume lužnjaka, topola i jasena u dolini reke (Ulmenion minoris) (leg. Milenković, M. et al., 15.12.2005., det. Milenković, M., BEO (Fungi) s.n.); Stig: Rašanac, UTM EQ22, 160 m, šume lužnjaka, topola i jasena u dolini reke (Ulmenion minoris) (leg. Milenković, M. et al. 24.10.1992., det. Milenković, M., BEO (Fungi) s.n.); Sr Morović, UTM CQ58, 80 $\mathrm{m}$, šume lužnjaka, topola i jasena u dolini reke (Ulmenion minoris), hrast lužnjak (leg. Lindtner, $V$, 17.10.1947., det. Lindtner, V, BEO (Fungi) s.n.); UTM CQ68, 80 m, šume lužnjaka, topola i jasena u dolini reke (Ulmenion minoris), hrast lužnjak (leg. Lindtner, $v$ 17.10.1947., det. Lindtner, V, BEO (Fungi) s.n.); Obedska bara: Obrež, UTM DQ25, 80 m, šume lužnjaka, topola i jasena u dolini reke 
(Ulmenion minoris), hrast lužnjak (leg. Milenković, M. et al. 15.10.2005., det. Milenković, M., BEO (Fungi) s.n.); Zemun: Boljevci, UTM DQ35, $80 \mathrm{~m}$, šume lužnjaka, topola i jasena u dolini reke (Ulmenion minoris) (leg. Lindtner, $V$ 25.10.1957., det. Lindtner, V, BEO (Fungi) s.n.); SZ Mačva: Bogatić, UTM CQ86, $80 \mathrm{~m}$, šume lužnjaka, topola i jasena u dolini reke (Ulmenion minoris) (leg. Milenković, M. et al., 06.11.1999., det. Milenković, M., BEO (Fungi) s.n.); 10.11.1998., det. Milenković, M., BEO (Fungi) s.n.); Šabac, Slepčević, UTM CQ85, 85 m, listopadna šuma (leg. Milenković, M. et al. 14.12.1996., det. Milenković, M., BEO (Fungi) s.n.); Tamnava: Ub, UTM DQ22, 100 m, listopadna šuma (leg. Milenković, M. et al. 15.12.2007., det. Milenković, M., BEO (Fungi) s.n.); Valjevo, UTM DQ10, 200 m, listopadna šuma, topola (leg. Milenković, M. et al. 28.11.2005., det. Milenković, M., BEO (Fungi) s.n.); šume lužnjaka, topola i jasena u dolini reke (Ulmenion minoris) (leg. Milenković, M. et al.

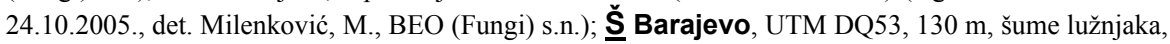
topola i jasena u dolini reke (Ulmenion minoris) (leg. Milenković, M. et al., 04.11.1998., det. Milenković, M., BEO (Fungi) s.n.); Batočina, UTM CQ68, $120 \mathrm{~m}$, šume lužnjaka, topola i jasena u dolini reke (Ulmenion minoris), hrast lužnjak (leg. Lindtner, $v$ 10.10.1947., det. Lindtner, V, BEO (Fungi) s.n.); Jasenica: Natalinci, UTM DP89, $100 \mathrm{~m}$, šume lužnjaka, topola i jasena u dolini reke (Ulmenion minoris), topola (leg. Milenković, M. et al. 12.11.1994., det. Milenković, M., BEO (Fungi) s.n.); Saranovo, UTM DP89, 130 m, šume lužnjaka, topola i jasena u dolini reke (Ulmenion minoris), topola (leg. Milenković, M. et al. 01.12.2001., det. Milenković, M., BEO (Fungi) s.n.); 04.11.1995., det. Milenković, M., BEO (Fungi) s.n.); 15.12.2005., det. Milenković, M., BEO (Fungi) s.n.); Kolubara: Lazarevac, Arnajevo, UTM DQ52, $200 \mathrm{~m}$, šume lužnjaka, topola i jasena u dolini reke (Ulmenion minoris) (leg. Milenković, M. et al. 04.11.1998., det. Milenković, M., BEO (Fungi) s.n.); Sokolovo, UTM DQ42, 90 m, šume lužnjaka, topola i jasena u dolini reke (Ulmenion minoris) (leg. Milenković, M. et al. 04.11.1998., det. Milenković, M., BEO (Fungi) s.n.); Obrenovac, UTM DQ34, $80 \mathrm{~m}$, šume lužnjaka, topola i jasena u dolini reke (Ulmenion minoris) (leg. Milenković, M. et al. 05.12.1995., det. Milenković, M., BEO (Fungi) s.n.); UTM DQ35, $75 \mathrm{~m}$, šume lužnjaka, topola i jasena u dolini reke (Ulmenion minoris), topola (leg. Milenković, M. et al. 06.12.2001., det. Milenković, M., BEO (Fungi) s.n.); Smederevo: Ralja, UTM DQ93, 100 m, šume lužnjaka, topola i jasena u dolini reke (Ulmenion minoris) (leg. Milenković, M. et al. 17.10.1998., det. Milenković, M., BEO (Fungi) s.n.); Vojvodina (leg. Milenkovíc, M. et al. 15.11.1992., det. Milenković, M., BEO (Fungi) s.n.).

\section{Agaricomycetes}

\section{HYMENOGASTRACEAE}

\section{Psilocybe serbica M.M. Moser \& E. Horak}

$\underline{Z}$ Tara planina, UTM CP76, 1080 m, mešovita šuma, ispod bukve (leg. Lindtner, V., Moser, M., 11.10.1963., det. Moser, M., BEO (Fungi) s.n.).

\section{AGARICACEAE}

\section{Battarrea phalloides (Dicks.) Pers.}

Bt Deliblatska peščara, UTM EQ16, 95 m, šumo-stepa (leg. Kus, B., 06.07.2016., det. Ivančević, B., BEO (Fungi) s.n.); I Niš, UTM EN79, 200 m, ruderalna livada u parku (leg. Kocić, M., 19.6.2014., det. Kustera, M., BEO (Fungi) s.n.). 


\section{AMANITACEAE}

Saproamanita vittadinii (Moretti) Redhead, Vizzini, Drehmel \& Contu

Š Beograd, UTM DQ56, 75 m, travnjak u parku (leg. Hadžić, I., 05.10.1998., det. Hadžić, I., BEO (Fungi) s.n., sub Amanita vittadinii).

\section{BOLETACEAE}

Butyriboletus regius (Krombh.) D. Arora \& J.L. Frank

SZ Cer, UTM CQ83, 330 m, hrastova šuma (leg. Hadžić, I., 09.10.2005., det. Hadžić, I., BEO (Fungi) s.n., sub Boletus regius); Valjevo, listopadna šuma (leg. Hadžić, I. 20.09.2001., det. Hadžić, I., BEO (Fungi) s.n., sub Boletus regius); $\underline{\mathbf{s}}$ Obrenovac: Mala Moštanica, UTM DQ44, $150 \mathrm{~m}$, listopadna šuma (leg. Hadžić, I., 27.05.1994., det. Hadžić, I., BEO (Fungi) s.n., sub Boletus regius).

Rubroboletus rhodoxanthus (Krombh.) Kuan Zhao \& Zhu L. Yang

SI Đerdap, listopadna šuma, verovatno hrast (leg. Jelić, M., 1964., det. Jelić, M., BEO (Fungi) s.n., sub Boletus rhodoxanthus).

Strobilomyces strobilaceus (Scop.) Berk.

C Cer, UTM CQ74, 155 m, hrastova šuma (leg. Hadžić, I., 14.10.2001., det. Hadžić, I., BEO (Fungi) s.n.); JI Vlasina, UTM FN03, 1500 m (leg. Hadžić, I., 21.09.2002., det. Hadžić, I., BEO (Fungi) s.n.).

\section{CANTHARELLACEAE}

\section{Cantharellus cibarius Fr.}

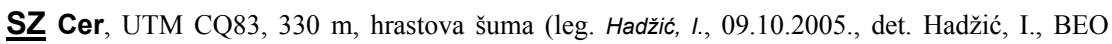
(Fungi) s.n.).

Cantharellus friesii Quél.

Š Beograd, UTM DQ55, 0 m, šuma kitnjaka i cera (leg. Hadžić, I., 15.07.1993., det. Hadžić, I., BEO (Fungi) s.n.).

\section{FOMITOPSIDACEAE}

Rhodofomes roseus (Alb. \& Schwein.) Kotl. \& Pouzar

C Kopaonik, 1600 m, smrčeva šuma, na smrči (leg. Lindtner, V., Moser, M., 21.10.1955., det. Lindtner, V., BEO (Fungi) s.n., sub Fomitopsis rosea). 
72 Jovanović, M. ET AL.: Plants, FUngi, AMPHiBIANS AND REPTILES FrOM SERBIA

\section{GEASTRACEAE}

Geastrum fornicatum (Huds.) Hook.

Bt Deliblatska peščara, UTM DQ97, $155 \mathrm{~m}$, borova (Pinus nigra) sađena šuma na pesku (leg. Ivančević, B., 10.1998., det. Ivančević, B., BEO (Fungi) s.n.).

\section{Geastrum melanocephalum (Czern.) V.J. Staněk}

I Niš, UTM EP70, 645 m, proplanak, livada (leg. Milenković, B., 25.09.2011., det. Kustera, M., BEO (Fungi) s.n.).

\section{Geastrum nanum Pers.}

Bt Deliblatska peščara, UTM EQ06, 145 m, sađeni bagrem (leg. Ivančević, B., 10.1998., det. Ivančević, B., BEO (Fungi) s.n., sub G. schmidelii).

\section{Myriostoma coliforme (Dicks.) Corda}

Š Beograd, UTM DQ55, 78 m, listopadna šuma lužnjaka, topola i jasena (leg. Radić, S., 1996., det. Radić, S., BEO (Fungi) s.n.).

\section{HERICIACEAE}

\section{Hericium cirrhatum (Pers.) Nikol.}

C Rudnik, UTM DP68, 1000 m, bukova šuma, na bukvi (leg. Ružanović, Ž., 12.6.2016., det. Ivančević, B., BEO (Fungi) s.n.).

\section{HYGROPHORACEAE}

Hygrophorus marzuolus (Fr.) Bres.

SI Đerdap, listopadna šuma, verovatno hrast (leg. Jelić, M., 1964., det. Jelić, M., BEO (Fungi) s.n.).

Hygrophorus russula (Schaeff. ex Fr.) Kauffman

SZ Cer, UTM CQ83, 330 m, hrastova šuma (leg. Hadžić, I., 09.10.2005., det. Hadžić, I., BEO (Fungi) s.n.).

\section{PhallaCeAE}

\section{Mutinus caninus (Huds.) Fr.}

SI Đerdap, bukova šuma (leg. Jelić, M., 1968., det. Jelić, M., BEO (Fungi) s.n.); SZ Cer, UTM CQ74, 155 m, hrastova šuma (leg. Hadžić, l., 14.10.2001., det. Hadžić, I., BEO (Fungi) s.n.);

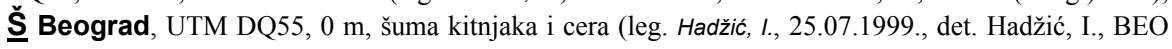
(Fungi) s.n.). 


\section{Phallus hadriani Vent.}

Bt Deliblatska peščara, UTM EQ07, 155 m, pašnjak (leg. Ivančević, B., 10.2009., det. Ivančević, B., BEO (Fungi) s.n.); UTM EQ26, 75 m, pašnjak (leg. Ivančević, B. 09.1994., det. Ivančević, B., BEO (Fungi) s.n.); Sr Zemun: Bečmen (leg. Hadžić, l., 11.09.1999., det. Hadžić, I., BEO (Fungi)

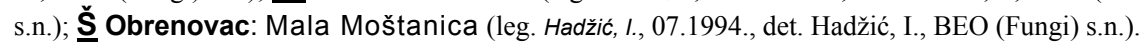

\section{POLYPORACEAE}

\section{Hapalopilus croceus (Pers.) Donk}

Š Avala, UTM DQ64, 400 m, hrastova šuma (leg. Ivančević, B., 1997., det. Ivančević, B., BEO (Fungi) s.n.

\section{AMPHIBIANS AND REPTILES}

\section{Amphibia}

\section{BOMBINATORIDAE}

\section{Bombina bombina (Linnaeus, 1761)}

Bt Kovin: Bavanište, UTM DQ96, 92 m (leg. Živković, M. 30.09.1984., det. Paunović, A., 108 SZV); Perlez: Sakule, UTM DQ59, 83 m (leg. Jovanović, M., 29.06.1988., det. Paunović, A., 182 SZV); Sr Novi Sad: Petrovaradin, Petrovaradinski rit, UTM DR10, 76 m (leg. Paunović, A., 11.10.1996., det. Paunović, A., 244 SZV); 76 m (leg. Paunović, A. 14.05.1995., det. Paunović, A., 347 SZV); 76 m (leg. Paunović, A. 26.03.1995., det. Paunović, A., 352 SZV); UTM DR11, 76 m (leg. Paunović, A. 30.09.1996., det. Paunović, A., 246 SZV); Petrovaradinski rit, Marija Snežna, UTM DR10, 75 m (leg. Paunović, A. 30.09.1996., det. Paunović, A., 245 SZV); $74 \mathrm{~m}$ (leg. Paunović, A.

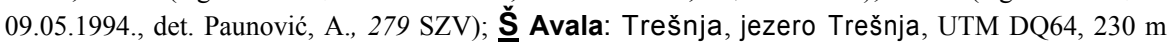
(leg. Paunović, A., 10.04.1998., det. Paunović, A., 854 SZV).

\section{Bombina variegata (Linnaeus, 1758)}

I Rtanj, UTM EP74, 732 m (leg. Paunović, A., 06.08.2006., det. Paunović, A., 910 SZV 140); SI Boljevac: Garje, UTM EP55, 670 m (leg. Paunović, A., 12.08.2005., det. Paunović, A., 896 SZV); Bor: Dubašnica, s. Bigerje, UTM EQ60, 750 m (leg. Paunović, A. 09.07.1995., det. Paunović, A., 707 SZV); s. Gornjane, UTM EP90, 380 m (leg. Paunović, A. 12.06.1994., det. Paunović, A., 405 SZV); Zlot, Zlotska klisura, UTM EP77 (leg. Paunović, A. 21.05.1994., det. Paunović, A., 406 SZV); (leg. Paunović, Milan 03.06.1997., det. Paunović, A., 824 SZV); UTM EP78 (leg. Paunović, A. 11.05.1996., det. Paunović, A., 413 SZV); Đerdap: Šomrda, Tatarski vis, UTM EQ73, $580 \mathrm{~m}$ (leg. Paunović, A. 04.08.2005., det. Paunović, A., 898 SZV); Južni Kučaj: Bele vode, UTM EP65, 700 m (leg. Paunović, A. 01.05.2008., det. Paunović, A., 917 SZV 147); Lukovo: Krivi Vir, UTM EP65, $350 \mathrm{~m}$ (leg. Paunović, M. 24.06.2004., det. Paunović, A., 881 SZV); SZ Valjevo: man. Ćelije, reka Gradac, UTM DP09 (leg. Koprivica, U., Koprivica, V., 10.04.1988., det. Paunović, A., 185 SZV); Z Tara planina: Zaovinsko jezero, UTM CP66, 900 m (leg. Paunović, A., 03.05.2005., det. Paunović, A., 887 SZV). 


\section{BUFONIDAE}

\section{Bufo bufo (Linnaeus, 1758)}

SI Boljetin: Boljetinska reka, UTM EQ83, 78 m (leg. Mučalica, M., 15.09.1884., det. Paunović, A., 935 SZV 165); Bor: s. Gornjane, UTM EP89, 440 m (leg. Paunović, A. 12.06.1994., det. Paunović, A., 404 SZV); Zlot, Zlotska klisura, UTM EP77 (leg. Paunović, Milan 03.06.1997., det. Paunović, A., 823 SZV); Donji Milanovac, UTM EQ92, 73 m (leg. Jovanović, M. 17.06.1985., det. Paunović, A., 126 SZV); Đerdap: Golubački grad, UTM EQ54 (leg. Jovanović, M. 13.06.1986., det. Paunović, A., 139 SZV); Tekija, UTM FQ14 (leg. Jovanović, M. 13.09.1984, det. Paunović, A., 103 SZV); Žagubica: vrelo Mlave, UTM EP69, 333 m (leg. Paunović, A. 18.05.2004., det. Paunović, A., 878 SZV); UTM EP69, 330 m (leg. Paunović, A. 05.08.2005., det. Paunović, A., 899 SZV); $\underline{\mathbf{s}}$ Topola: Krćevac, UTM DQ70, 160 m (leg. Paunović, A., 30.10.2002., det. Paunović, A., 871 SZV).

\section{Bufotes viridis (Laurenti, 1768)}

Bč Horgoš: Mala pijaca, UTM DS10, 88 m (leg. Paunović, A., 22.07.1997., det. Paunović, A., 847 SZV, sub Bufo viridis); Srbobran: Miodragovićev salaš, UTM DR02, $80 \mathrm{~m}$ (leg. Paunović, A. 20.08.2005., det. Paunović, A., 901 SZV, sub Bufo viridis); Bt Kovin: Bavanište, UTM DQ96 (leg. Živković, M., 28.09.1984., det. Paunović, A., 107 SZV, sub Bufo viridis); Vršac: Gaj, UTM ER11, 83 m (leg. Paunović, A. 20.08.2006., det. Paunović, A., 911 SZV 141, sub Bufo viridis); ! Bovan: Bovansko jezero, UTM EP53, $230 \mathrm{~m}$ (leg. Paunović, A., 25.06.2002., det. Paunović, A., 864 SZV, sub Bufo viridis); Po Požarevac: Mala Krsna, UTM EQ03, 76 m (leg. Paunović, A., 23.09.1984., det. Paunović, A., 114 SZV, sub Bufo viridis); (leg. Jovanović, M. 09.07.1984., det. Paunović, A., 91 SZV 99, sub Bufo viridis); SI Bor: Žagubica, r. Tisnica, UTM EP69, 320 m (leg. Paunović, A., 12.06.1994., det. Paunović, A., 798 SZV, sub Bufo viridis); Sr Beograd: Blok 45, UTM DQ56, 76 m (leg. Jovanović, M., 07.07.1987., det. Paunović, A., $163 \mathrm{SZV}$, sub Bufo viridis); $\underline{\mathbf{S}}$ Avala: Trešnja, jezero Trešnja, UTM DQ67, 230 m (leg. Vlajić, A., 10.04.1998., det. Paunović, A., 858 SZV 132).

\section{HYLIDAE}

\section{Hyla arborea (Linnaeus, 1758)}

JZ Prijepolje: Mileševo, UTM CP90, 760 m (leg. Paunović, A., 10.05.1997., det. Paunović, A., $649 \mathrm{SZV})$.

\section{PELOBATIDAE}

\section{Pelobates fuscus (Laurenti, 1768)}

Bt Beograd: Krnjača, UTM DQ56, 71 m (leg. Paunović, A., 13.04.2000., det. Paunović, A., 925 SZV 155); Deliblatska peščara: Utrine, Dabin salaš, UTM EQ26, $73 \mathrm{~m}$ (leg. Paunović, $A$. 09.08.1999., det. Paunović, A., 923 SZV 153); Sr Novi Sad: Petrovaradin, Petrovaradinski rit, UTM DR11, 76 m (leg. Paunović, A., 21.08.1996., det. Paunović, A., 719 SZV).

\section{Pelobates syriacus Boettger, 1889}

Bt Deliblatska peščara: Hatarica, UTM EQ70, $102 \mathrm{~m}$ (leg. Paunović, A., 23.04.2004., det. Paunović, A., 873 SZV); Utrine, UTM EQ26 (leg. Paunović, A. 21.04.2000., det. Paunović, A., 926 SZV 156). 


\section{RANIDAE}

\section{Pelophylax esculentus (Linnaeus, 1758)}

Bt Pančevo: Pančevački rit, UTM DQ66, 76 m (leg. Paunović, A., 16.07.2004., det. Paunović, A., 883 SZV, sub Rana kl. esculenta); 71 m (leg. Paunović, A. 16.07.2004., det. Paunović, A., 884 SZV, sub Rana kl. esculenta); Sr Novi Sad: Petrovaradin, Petrovaradinski rit, UTM DR10, 76 m (leg. Paunović, A., 14.05.1995., det. Paunović, A., 345 SZV, sub Rana kl. esculenta); 80 m (leg. Paunović, A. 30.09.1996., det. Paunović, A., 229 SZV, sub Rana kl. esculenta); 76 m (leg. Paunović, A. 09.05.1994., det. Paunović, A., 297 SZV, sub Rana kl. esculenta); UTM DR11, 75 m (leg. Paunović, A. 30.09.1996., det. Paunović, A., 207 SZV, sub Rana kl. esculenta); 76 m (leg. Paunović, A. 07.09.1995., det. Paunović, A., $436 \mathrm{SZV}$, sub Rana kl. esculenta).

\section{Pelophylax lessonae Camerano, 1882}

Sr Novi Sad: Petrovaradin, Petrovaradinski rit, UTM DR10, $76 \mathrm{~m}$ (leg. Paunović, A., 30.09.1996., det. Paunović, A., 208 SZV, sub Rana lessonae); 76 m (leg. Paunovíc, A. 13.06.1995., det. Paunović, A., 332 SZV, sub Rana lessonae); 76 m (leg. Paunović, A. 07.08.1995., det. Paunović, A., 737 SZV, sub Rana lessonae); UTM DR11, 76 m (leg. Paunović, A. 11.10.1996., det. Paunović, A., 236 SZV, sub Rana lessonae); Petrovaradinski rit, Marija Snežna, UTM DR10, $77 \mathrm{~m}$ (leg. Paunović, A. 30.09.1996., det. Paunović, A., 230 SZV, sub Rana lessonae); 77 m (leg. Paunović, A. 11.10.1996., det. Paunović, A., 241 SZV, sub Rana lessonae); 76 m (leg. Paunović, A. 09.05.1994., det. Paunović, A., 310 $\mathrm{SZV}$, sub Rana lessonae).

\section{Pelophylax ridibundus Pallas, 1771}

Bč Odžaci: Ratkovo, UTM CR63, 85 m (leg. Jakov, M., 31.05.1989., det. Paunović, A., 193 SZV, sub Rana ridibunda); I Aleksinac: Suva bara, UTM EP41, $290 \mathrm{~m}$ (leg. Paunović, A., 24.06.2002., det. Paunović, A., 863 SZV, sub Rana ridibunda); Sićevačka klisura: s. Ostrovica, Ostrovička Banjica, UTM EN99, 400 m (leg. Paunović, A. 08.07.1994., det. Paunović, A., 265 SZV, sub Rana ridibunda); s. Sićevo, Nišava, UTM EN89, 210 m (leg. Paunović, A. 08.07.1994., det. Paunović, A., 267 SZV, sub Rana ridibunda); Po Velika Plana: Petrovac na Mlavi, UTM EQ31, $120 \mathrm{~m}$ (leg. Paunović, A., 18.05.2004., det. Paunović, A., 879 SZV); SI Bor: Bor, Borsko jezero, UTM EP88, 450 m (leg. Paunović, A., 10.06.1994., det. Paunović, A., 389 SZV, sub Rana ridibunda); s. Bučje, pl. Stol, UTM EP99, 900 m (leg. Olivera Bjelić 24.08.1996., det. Paunović, A., 839 SZV, sub Rana ridibunda); s. Gornjane, UTM EP89, 350 m (leg. Paunović, A. 12.06.1994., det. Paunović, A., 401 SZV, sub Rana ridibunda); Zlot, UTM EP77, 265 m (leg. Paunović, A. 21.08.1994., det. Paunović, A., 263 SZV, sub Rana ridibunda); 280 m (leg. Paunović, A. 21.09.1995., det. Paunović, A., 382 SZV, sub Rana ridibunda); Đerdap: Brnjica, Brnjička reka, UTM EQ64 (leg. Jovanović, M. 13.06.1986., det. Paunović, A., 141 SZV, sub Rana ridibunda); Golubac, UTM EQ54 (leg. Jovanović, M. 13.06. 1986., det. Paunović, A., 140 SZV, sub Rana ridibunda); (leg. Jovanović, M. 10.04.1988., det. Paunović, A., 933 SZV 163, sub Rana ridibunda); Pecka bara, UTM FQ04, 89 m (leg. anonim. 18.10.1967., det. Paunović, A., 81 SZV 91, sub Rana ridibunda); Sr Novi Sad: Petrovaradin, Petrovaradinski rit, UTM DR10, 76 m (leg. Paunović, A., 13.06.1995., det. Paunović, A., 329 SZV, sub Rana ridibunda); 76 m (leg. Paunović, A. 14.05.1995., det. Paunović, A., 346 SZV, sub Rana ridibunda); 76 m (leg. Paunović, A. 12.07.1995., det. Paunović, A., 418 SZV, sub Rana ridibunda); 76 m (leg. Paunović, A. 07.09.1995., det. Paunović, A., 438 SZV, sub Rana ridibunda); Petrovaradinski rit, Marija Snežna, UTM DR10, 76 m (leg. Paunović, A. 09.05.1994., det. Paunović, A., 282 SZV, sub Rana ridibunda); $\underline{\mathbf{S Z}}$ Valjevo: man. Ćelije, reka Gradac, UTM DP09, 250 m (leg. Koprivica, U., 10.04.1988., det. Paunović, A., 933

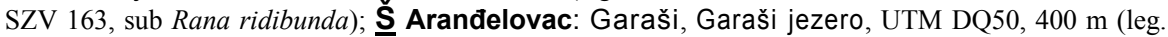
Paunović, A., 29.10.2002., det. Paunović, A., 866 SZV, sub Rana ridibunda); Avala: Trešnja, jezero Trešnja, UTM DQ66, 230 m (leg. Vlajić, A. 10.04.1998., det. Paunović, A., 857 SZV 131); Knić: Gružansko jezero, UTM DP76, 300 m (leg. Paunović, A. 27.04.2004., det. Paunović, A., 875 SZV); 
Topola: Krćevac, UTM DQ70, 160 m (leg. Paunović, A. 30.10.2002., det. Paunović, A., 869 SZV, sub Rana ridibunda).

\section{Rana dalmatina Bonaparte, 1840}

Bt Pančevački rit: Krnjača, UTM DQ66 (leg. Paunović, A., 27.10.2005., det. Paunović, A., 903 SZV); Reva, UTM DQ66, 76 m (leg. Paunović, A. 27.10.2005., det. Paunović, A., 903 SZV); C PI. Radan: s. Ivanje, UTM EN46, 750 m (leg. Paunović, A., 23.07.1994., det. Paunović, A., 318 SZV); s. Obražda, UTM EN45, 864 m (leg. Paunović, A. 25.07.1994., det. Paunović, A., 316 SZV); Šopot, UTM EN46, 1300 m (leg. Paunović, A. 24.07.1994., det. Paunović, A., 315 SZV); SI Boljevac: Garje, UTM EP55, 670 m (leg. Paunović, A., 12.08.2005., det. Paunović, A., 894 SZV); Bor: Zlot, blizu Mandine pećine, UTM EP77, 403 m (leg. Paunović, A. 20.08.1994., det. Paunović, A., 261 SZV); Zlotska klisura, UTM EP77 (leg. Paunović, Milan 03.06.1997., det. Paunović, A., 826 SZV); UTM EP78, 300 m (leg. Paunović, A. 10.05.1996., det. Paunović, A., 417 SZV); Žagubica, Beljevinska reka, okolina, UTM EP77, 255 m (leg. Paunović, A. 11.06.1994., det. Paunović, A., 402 SZV); Đerdap: Boljetin, Boljetinska reka, UTM EQ83, 78 m (leg. Jovanović, M. 18.06.1985., det. Paunović, A., 127 SZV); Dobra, Leva reka, UTM EQ74 (leg. anonim. 23.10.1967., det. Paunović, A., 82 SZV 92); Lukovo: Krivi Vir, UTM EP65 (leg. Paunović, A. 09.09.2004., det. Paunović, A., 886 SZV); Sr Novi Sad: Petrovaradin, Petrovaradinski rit, UTM DR10, 75 m (leg. Paunovíc, A., 30.06.1995., det. Paunović, A., 325 SZV); 76 m (leg. Paunović, A. 12.07.1995., det. Paunović, A., 327 SZV); UTM DR11, 76 m (leg. Paunović, A. 26.03.1995., det. Paunović, A., 293 SZV); 76 m (leg. Paunović, A. 12.07.1996, det. Paunović, A., 323 SZV); 76 m (leg. Paunović, A. 23.05.1997., det. Paunović, A., 789 SZV); Petrovaradinski rit, Marija Snežna, UTM DR10, 75 m (leg. Paunović, A. 09.05.1994., det. Paunović, A., 283 SZV); 75 m (leg. Paunović, A. 11.07.1996., det. Paunović, A., 320 SZV); $\underline{\text { SZ }}$ Sremska Mitrovica: Zasavica, potok Batar, UTM CQ77, 81 m (leg. Paunović, A., 03.11.2005., det. Paunović, A., $904 \mathrm{SZV}$ ); $\underline{\mathbf{s}}$ Avala: Trešnja, jezero Trešnja, UTM DQ65, $230 \mathrm{~m}$ (leg. Vlajić, A., 10.04.1998., det. Paunović, A., 855 SZV 129); Obrenovac: Zabran, UTM DQ34, 73 m (leg. Kašić. Lj. 23.07.1978., det. Paunović, A., 944 SZV).

\section{Rana graeca Boulenger, 1891}

SZ Valjevo: reka Jablanica, UTM CP99 (leg. Paunović, M., 20.04.1992., det. Paunović, A., 195 SZV); Z Kraljevo: Studenica, r. Studenica, UTM DP61, 500 m (leg. Paunović, A., 02.08.1994., det. Paunović, A., 811 SZV); (leg. Paunović, A. 08.08.1994., det. Paunović, A., 817 SZV).

\section{Rana temporaria Linnaeus, 1758}

K Šar-planina: Brezovica, UTM EM07, 1610 m (leg. Paunović, A., 16.07.1995, det. Paunović, A., 633 SZV); UTM EM08, 1460 m (leg. Paunović, A. 16.07.1996, det. Paunović, A., 634 SZV); Crni kamen, UTM EM06, 2200 m (leg. Paunović, A. 20.07.1995., det. Paunović, A., 640 SZV); Durlov potok, UTM EM07, $1800 \mathrm{~m}$ (leg. Paunović, A. 20.07.1995., det. Paunović, A., 639 SZV); M Šarplanina: Restelica, Kula crnkamenska, UTM DM73, 1900 m (leg. Voislav Vasić 09.07.1997., det. Paunović, A., 842 SZV); SI Boljevac: Javorak, UTM EP56, 800 m (leg. Paunović, A., 03.08.2005., det. Paunović, A., 895 SZV); Bor: Zlot, UTM EP77, 265 m (leg. Paunović, A. 21.08.1994., det. Paunović, A., 262 SZV); Južni Kučaj: Bele vode, UTM EP65, 700 m (leg. Paunović, A. 01.05.2008., det. Paunović, A., 915 SZV 145). 


\section{SALAMANDRIDAE}

\section{Ichthyosaura alpestris (Laurenti, 1768)}

Z Tara planina: Mitrovac, UTM CP76, 1080 m (leg. Paunović, A. 03.05.2005., det. Paunović, A., $891 \mathrm{SZV}$, sub Triturus alpestris); Zaovinsko jezero, UTM CP66, $900 \mathrm{~m}$ (leg. Paunović, A., 03.05.2005., det. Paunović, A., 887 SZV, sub Triturus alpestris).

\section{Lissotriton vulgaris (Linnaeus, 1758)}

J Preševo, UTM EM58 (leg. Puzović, S., 08.07.1988., det. Paunović, A., 190 SZV, sub Triturus vulgaris); SI Boljevac: Garje, UTM EP55, 670 m (leg. Paunović, A., 12.08.2005., det. Paunović, A., 900 SZV, sub Triturus vulgaris); Južni Kučaj: Bele vode, UTM EP65, 700 m (leg. Paunović, A. 01.05.2008., det. Paunović, A., 918 SZV 148); Š Beograd: Košutnjak, kafana Košuta, UTM DQ55, 130 m (leg. Koprivica, U., Koprivica, V., 04.04.1988., det. Paunović, A., 184 SZV, sub Triturus vulgaris); Z Tara planina: Mitrovac, UTM CP76, $1080 \mathrm{~m}$ (leg. Paunović, A., 03.05.2005., det. Paunović, A., 893 SZV, sub Triturus vulgaris).

\section{Salamandra salamandra (Linnaeus, 1758)}

I Aleksinac: Suva bara, UTM EP41, 290 m (leg. Paunović, A., 24.06.2002., det. Paunović, A., 862 SZV); Ozren: Ripaljka, UTM EP63, 420 m (leg. Paunović, A. 31.05.2001., det. Paunović, A., 929 SZV 159); Zaječar: s. Vratarnica, pećina Toplik, UTM FP04, 198 m (leg. Velojić 18.03.1997., det. Paunović, A., 381 SZV); SI Bor: Zlot, blizu Mandine pećine, UTM EP77, 403 m (leg. Paunović, Milan, 1998., det. Paunović, A., 850 SZV); Đerdap: Boljetin, Boljetinska reka, UTM EQ83 (leg. Jovanović, M. 15.09.1984., det. Paunović, A., 106 SZV); Tekija, mali Kovilovski potok, UTM FQ14 (leg. Jovanović, M. 14.07.1998., det. Paunović, A., 178 SZV); Vlaški potok, UTM FQ04 (leg. Jovanović, M. 13.07.1998., det. Paunović, A., 177 SZV); Turski potok, UTM EQ64 (leg. Jovanović, M. 14.06. 1986, det. Paunović, A., 938 SZV 168, sub Salamandra salamandra); Južni Kučaj: Bele vode, UTM EP65, 700 m (leg. Paunović, A. 01.05.2008., det. Paunović, A., 916 SZV 146); Lukovo: Krivi Vir, UTM EP65, 414 m (leg. Paunović, A. 10.06.2000., det. Paunović, A., 860 SZV); SZ Valjevo: man. Ćelije, reka Gradac, UTM DP09, 240 m (leg. Koprivica, U., 10.04.1988., det. Paunović, A., 934 SZV 164); $\underline{\text { S } A r a n đ e l o v a c: ~ b r d o ~ R i s o v a c ̌ a, ~ p e c ́ i n a ~ R i s o v a c ̌ a, ~ U T M ~ D Q 60, ~} 0$ m (leg. Paunović, A., 26.10.2018., det. Paunović, A., 948 SZV); Avala: Trešnja, jezero Trešnja, UTM DQ63, 230 m (leg. Vlajić, A. 10.04.1998., det. Paunović, A., 853 SZV); Knić: Gružansko jezero, potok Ćurevac, UTM DP76, 275 m (leg. Paunović, A. 28.04.2004., det. Paunović, A., 875 SZV); Z Guča: Gornj Dubovac, UTM DP43, 900 m (leg. Paunović, A., 30.04.2009., det. Paunović, A., 920 SZV 150).

\section{Reptilia}

\section{ANGUIDAE}

\section{Anguis fragilis Linnaeus, 1758}

C PI. Radan, UTM EN46, 735 m (leg. Paunović, A., 24.07.1994., det. Paunović, A., 363 SZG); Sr Novi Sad: Petrovaradin, Petrovaradinski rit, UTM DR10, 76 m (leg. Paunović, A., 11.10.1995., det. Paunović, A., 346 SZG); 75 m (leg. Paunović, A. 22.05.1996., det. Paunović, A., 347 SZG); 75.5 m (leg. Paunović, A. 30.09.1996., det. Paunović, A., 357 SZG); UTM DR11, 76 m (leg. Paunović, A. 11.07.1996., det. Paunović, A., 348 SZG); Š Beograd: Leštane, UTM DQ65, 150 m (leg. Paunović, A., 11.04.1998., det. Paunović, A., 442 SZG 221). 


\section{COLUBRIDAE}

\section{Coronella austriaca Laurenti, 1768}

C Goč: Krnja jela, UTM DP82 (leg. Paunović, A., 1999., det. Paunović, A., 448 SZG); $\underline{\text { SI Bor: }}$ Borsko jezero, UTM EP88, 445 m (leg. Paunović, A., 10.06.1994., det. Paunović, A., 335 SZG 376).

\section{Dolichophis caspius (Gmelin, 1789)}

C Prokuplje: Dubovo, Asanovac, UTM EN57, 750 m (leg. Paunović, A., 12.12.2006., det. Paunović, A., 467 SZG 335); I Sićevačka klisura: s. Sićevo, r. Nišava, UTM EN89, 215 m (leg. Paunović, A., 09.07.1994., det. Paunović, A., 340 SZG 381, sub Coluber caspius); SI Bor: s. Brestovac, Brestovačka Banja, UTM EP87, 385 m (leg. Paunović, A., 24.09.1995., det. Paunović, A., 337 SZG 378, sub Coluber caspius); Zlot, UTM EP77, 280 m (leg. Paunović, A. 09.06.1994., det. Paunović, A., 349 SZG 387, sub Coluber caspius).

\section{Natrix natrix (Linnaeus, 1758)}

SI Lukovo: Krivi Vir, UTM EP65 (leg. Paunović, A., 02.08.2006., det. Paunović, A., 463 SZG 331); Sr Novi Sad: Fruška gora, Popovičko jezero, UTM DR00, $250 \mathrm{~m}$ (leg. Paunović, A., 30.09.1996., det. Paunović, A., 460 SZG); Petrovaradin, Petrovaradinski rit, UTM DR10, $76 \mathrm{~m}$ (leg. Paunović, A. 28.09.1995., det. Paunović, A., 336 SZG 377); 76 m (leg. Paunović, A. 30.09.1996., det. Paunović, A., 358 SZG); UTM DR11, 75 m (leg. Paunović, A. 30.09.1996., det. Paunović, A., 360 SZG).

\section{Natrix tessellata (Laurenti, 1768)}

I Sićevačka klisura: s. Sićevo, r. Nišava, UTM EN89, 210 m (leg. Paunović, A., 08.07.1994., det. Paunović, A., 339 SZG 380); SI Boljevac: Mali Izvor, UTM EP75, 305 m (leg. Paunović, A., 07.12.2006., det. Paunović, A., 465 SZG 333); Sr Novi Sad: Petrovaradin, Petrovaradinski rit, UTM DR10, 74.8 m (leg. Paunović, A., 30.05.1995., det. Paunović, A., 352 SZG 389); UTM DR11, 76 m

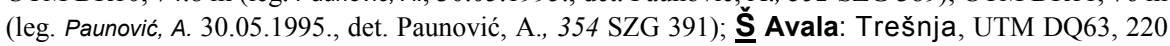
m (leg. Paunović, A., 11.04.1998., det. Paunović, A., 440 SZG 319); Knić: Gružansko jezero, UTM DP76, $200 \mathrm{~m}$ (leg. Paunović, A. 28.04.2004., det. Paunović, A., 454 SZG 341); $\underline{\mathbf{Z}}$ Kraljevo: r. Studenica, man. Studenica, UTM DP61, 480 m (leg. Paunović, A., 06.08.1994., det. Paunović, A., 374 SZG).

\section{Zamenis longissimus (Laurenti, 1768)}

Bt Pančevo: Reva, UTM DQ66, 76 m (leg. Paunović, A., 26.04.2006., det. Paunović, A., 462 SZG 330); SI Boljevac: Mali Izvor, UTM EP75, 305 m (leg. Paunović, A., 07.12.2006., det. Paunović, A., 466 SZG 334); Bor: Zlot, kanjon Lazareve reke, UTM EP77, $280 \mathrm{~m}$ (leg. Paunović, A. 11.05.1996., det. Paunović, A., 334 SZG 375, sub Elaphe longissima); Sr Novi Sad: Petrovaradin, Petrovaradinski rit, UTM DR10, 76 m (leg. Paunović, A., 11.10.1995., det. Paunović, A., 345 SZG 386,

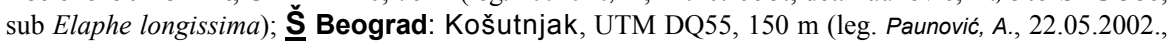
det. Paunović, A., 451 SZG 394, sub Elaphe longissima).

\section{EMYDIDADE}

\section{Emis orbicularis (Linnaeus, 1758)}

Bč Horgoš: Mala pijaca, UTM DS10, 90 m (leg. Paunović, A., 22.07.1997., det. Paunović, A., $431 \mathrm{SZG})$. 


\section{LACERTIDAE}

\section{Lacerta agilis Linnaeus, 1758}

Bč Horgoš: Selevenjske pustare, UTM DS11, 96 m (leg. Paunović, A., 22.07.1997., det. Paunović, A., $430 \mathrm{SZG}$ ); Bt Čenta: Čenćanski kanal, UTM DQ59, $77 \mathrm{~m}$ (leg. Paunović, A., 26.03.1994., det. Paunović, A., 429 SZG); Sr Novi Sad: Petrovaradin, Petrovaradinski rit, UTM DR11, 76 m (leg. Paunović, A., 30.09.1996., det. Paunović, A., 355 SZG); 75.9 m (leg. Paunović, A. 30.06.1995., det. Paunović, A., 361 SZG).

\section{Lacerta viridis (Laurenti, 1768)}

I Sićevačka klisura: s. Sićevo, Vis, UTM EN89, 365 m (leg. Paunović, A., 14.07.1994., det. Paunović, A., 306 SZG); Sokobanja: Ozren, UTM EP63, 500 m (leg. Paunović, A. 06.08.2006., det. Paunović, A., 464 SZG 332); Zaječar: Grljan, Grljanski krš, UTM FP05, $170 \mathrm{~m}$ (leg. Paunović, A. 03.06.1997., det. Paunović, A., 422 SZG); SI Bor: Zlot, kanjon Lazareve reke, UTM EP77 (leg. Paunović, A., 09.07.1995., det. Paunović, A., 319 SZG); (leg. Paunović, A. 09.07.1995., det. Paunović, A., 320 SZG); (leg. Paunović, A. 11.05.1996., det. Paunović, A., 324 SZG); Lukovo: Krivi Vir, UTM EP65, 400 m (leg. Paunović, A. 04.07.2005., det. Paunović, A., 459 SZG); 亭 Knić: Gružansko jezero, UTM DP76, 280 m (leg. Paunović, A., 27.04.2004., det. Paunović, A., 455 SZG).

\section{Podarcis muralis (Laurenti, 1768)}

I Sićevačka klisura: s. Ostrovica, Ostrovička Banjica, UTM EN99, $400 \mathrm{~m}$ (leg. Paunović, A. 08.07.1994., det. Paunović, A., 303 SZG); UTM EN99, 275 m (leg. Paunović, A. 08.07.1994., det. Paunović, A., 302 SZG); s. Sićevo, UTM EN89, 460 m (leg. Paunović, A. 13.07.1994., det. Paunović, A., 305 SZG); Stara planina: Crni vrh, UTM FN38, 1150 m (leg. Paunović, A. 20.07.1992., det. Paunović, A., 400 SZG); Kalna, UTM FP10, 463 m (leg. Paunović, A. 15.07.1992., det. Paunović, A., 399 SZG); Krajište, UTM FN48, 880 m (leg. Paunović, A. 16.07.1992., det. Paunović, A., 388 SZG); Topli Do, UTM FP30, 800 m (leg. Paunović, A. 12.07.1992., det. Paunović, A., 375 SZG); UTM FP31, 900 m (leg. Paunović, A. 12.07.1992., det. Paunović, A., 376 SZG); Vidlič, Gostuška reka, UTM FN38, 800 m (leg. Paunović, A. 20.07.1992., det. Paunović, A., 398 SZG); Zavoj, Šenički rt, UTM FN39, 813 m (leg. Paunović, A. 15.07.1992., det. Paunović, A., 384 SZG); UTM FN39, $650 \mathrm{~m}$ (leg. Paunović, A. 20.07.1992., det. Paunović, A., 397 SZG); JZ Prijepolje: kanjon Mileševke, UTM CP90, 620 m (leg. Paunović, A., 12.05.1997., det. Paunović, A., 368 SZG); Uvac: Nemanjin grad, UTM DP10 (leg. Paunović, A. 12.05.1997., det. Paunović, A., 367 SZG); SI Bor: Zlot, Demižlok, UTM EP77, 950 m (leg. Paunović, A., 01.04.1994., det. Paunović, A., 308 SZG); kanjon Lazareve reke, UTM EP77, 320 m (leg. Paunović, A. 01.04.1994., det. Paunović, A., 309 SZG); 420 m (leg. Paunović, A. 09.07.1995., det. Paunović, A., 310 SZG); (leg. Paunović, A. 09.07.1995., det. Paunović, A., 316 SZG); 380 m (leg. Paunović, A. 11.05.1996., det. Paunović, A., 321 SZG); (leg. Paunović, A. 11.05.1996., det. Paunović, A., 322 SZG); r. Mikuljska reka, UTM EP77, $250 \mathrm{~m}$ (leg. Paunović, A.

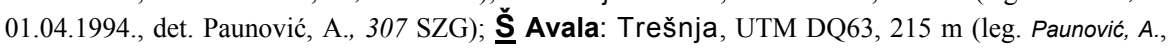
11.04.1998., det. Paunović, A., 441 SZG 220); Z Kraljevo: Studenica, man. Studenica, 480 m (leg. Paunović, A., 08.08.1994., det. Paunović, A., 373 SZG).

\section{Zootoca vivipara (Lichtenstein, 1823)}

K Šar-planina: Brezovica, Durlov potok, UTM EM06, 1450 m (leg. Paunoví, A., 20.07.1995., det. Paunović, A., 428 SZG, sub Lacerta vivipara). 
80 Jovanović, M. ET AL.: PlANTS, FUngI, AMPHIBIANS AND REPTILES FROM SERBIA

\section{VIPERIDAE}

Vipera ammodytes (Linnaeus, 1758)

I Rtanj: rudnik Rtanj, UTM EP74, 650 m (leg. Paunović, A., 24.09.1995., det. Paunović, A., 342 SZG 383); Sićevačka klisura: s. Ostrovica, Ostrovička Banjica, UTM EN99, $500 \mathrm{~m}$ (leg. Paunović, A. 08.07.1994., det. Paunović, A., 344 SZG 385); SI Bor: Zlot, p. Beljevina, UTM EN89, 210 m (leg. Paunović, A., 11.06.1994., det. Paunović, A., 341 SZG 382).

Vipera berus (Linnaeus, 1758) subsp. bosniensis Boettger, 1889

K Šar-planina: Brezovica, UTM EM07, 2200 m (leg. Paunović, A., 29.07.1995., det. Paunović, A., $350 \mathrm{SZG})$.

\section{DISCUSSION}

\section{VASCULAR PLANTS}

In the database of Polypodiopsida there are 34 specimens in total, classified in 4 families: Athyriaceae 23 (67.65\%), with single genus and single species, Ophioglossaceae 7 (20.59\%), with single genus and single species, Marsileaceae 2 (5.88 \%), with single genus and single species and Salviniaceae (Azollaceae) 2 (5.88 \%), with single genus and single species.

In the database of Pinopsida there are 32 specimens in total, classified in 2 families: PINACEAE 28 (87.50\%), with single genus and 3 species and TAXACEAE 4 (12.50\%), with single genus and single species.

In the database of Liliopsida there are 210 specimens in total, classified in 6 families: LiLIACEAE (LiLIACEAE) $81(38.57 \%$ ), with 2 genera and 4 species, LILIACEAE (ASPARAGACEAE) $50(23.81 \%$ ), with 2 genera and 3 species, LILIACEAE (AMARYLLIDACEAE) $48(22.86 \%$ ), with 2 genera and 2 species, LiLIACEAE (MELANTHIACEAE) 29 (13.81\%), with single genus and 2 species, ACORACEAE single $(0.48 \%)$, with single genus and single species and HYDROCHARITACEAE single $(0.48 \%)$, with single genus and single species.

In the database of Magnoliopsida there are 560 specimens in total, classified in 24 families: GENTIANACEAE 78 (13.93\%), with 2 genera and 5 species, RANUNCULACEAE 67 (11.96\%), with 3 genera and 7 species, CARYOPHYLLACEAE 58 (10.36\%), with 7 genera and 11 species, GERANIACEAE $49(8.75 \%)$, with 2 genera and 5 species, Plumbaginaceae 49 $(8.75 \%)$, with 4 genera and 4 species, MALVACEAE (TILIACEAE) 36 (6.43 $\%$ ), with single genus and 2 species, BETULACEAE (CORYLACEAE) 29 (5.18 $\%$ ), with single genus and single species, BRASSICACEAE (CRUCIFERAE) 27 (4.82\%), with 5 genera and 7 species, ARISTOLOCHIACEAE 25 (4.46\%), with single genus and single species, SAXIFRAGACEAE 19 (3.39\%), with 
single genus and 2 species, Fagaceae 16 (2.86\%), with single genus and single species, BetUlACEAE (BETULACEAE) 12 (2.14\%), with single genus and single species, RHAMNACEAE $12(2.14 \%)$, with single genus and single species, RUTACEAE 12 (2.14 \%), with 2 genera and 2 species, MALVACEAE (MALVACEAE) 11 (1.96\%), with 2 genera and 2 species, PAEONIACEAE 11 (1.96\%), with single genus and 2 species, Urticaceae 11 $(1.96 \%$ ), with 2 genera and 2 species, AMARANTHACEAE (CHENOPODIACEAE) 9 (1.61\%), with 2 genera and 2 species, ROSACEAE $9(1.61 \%)$, with 2 genera and 2 species, GROSSULARIACEAE 6 (1.07\%), with single genus and 2 species, EUPHORBIACEAE 5 (0.89\%), with single genus and 2 species, ULMACEAE $5(0.89 \%)$, with single genus and single species, CistaCEAe $2(0.36 \%)$, with 2 genera and 2 species, NYMPHAEACEAE 2 $(0.36 \%)$, with single genus and single species.

The database contains 86 species of vascular plants which are protected (58) and strictly protected (28) in Serbia based on "The rulebook on the proclamation and protection of strictly protected and protected wild species of plants, animals and fungi" (Institute for nature conservation of Serbia). Only three species, Armeria rumelica Boiss., Salvinia natans (L.) All. and Spiraea cana Waldst. \& Kit., are not protected by this rulebook. Four taxa, Marsilea quadrifolia L., Galanthus nivalis L., Ruscus aculeatus L. and Gentiana lutea L. subsp. symphyandra (Murb.) Hayek, are protected by the Habitats directive, while four taxa, Marsilea quadrifolia, Fritillaria montana Hoppe ex W. D. J. Koch, Lilium carniolicum Bernh. ex W. D. J. Koch subsp. jankae (A. Kern.) Asch. \& Graebn. and Salvinia natans (L.) All. are protected by Bern convention. In addition Galanthus nivalis and Gentiana lutea subsp. symphyandra are CITES species.

\section{FUNGI}

In the database of Pezizomycetes there are 127 specimens in total, classified in 2 families: TUBERACEAE 123 (96.85\%), with single genus and 3 species and MORCHELlaCEAE 4 (3.15\%), with single genus and 2 species.

In the database of Agaricomycetes there are 28 specimens in total, classified in 11 families: PHALlaceAe 7 (25\%), with 2 genera and 2 species, BOLETACEAE $6(21.43 \%)$, with 3 genera and 3 species, GEASTRACEAE $4(14.29 \%)$, with 2 genera and 4 species, AgARICACEAE $2(7.14 \%)$, with single genus and single species, CANTHARELLACEAE 2 (7.14 \%), with single genus and 2 species, HYGROPHORACEAE 2 (7.14 \%), with single genus and 2 species, AMANITACEAE single (3.57\%), with single genus and single species, FOMITOPSIDACEAE single $(3.57 \%)$, with single genus and single species, HERICIACEAE single (3.57\%), with single genus and single 
species, HyMENOGASTRACEAE single (3.57\%), with single genus and single species, POLYPORACEAE single $(3.57 \%)$, with single genus and single species.

Data on specimens of protected and strictly protected fungi species in the Republic of Serbia, deposited in the BEO collection, have been extracted and presented. These records are provided for species from 18 genera, with most specimens from hypogeic fungi of the genus Tuber. The findings include the oldest ones from 1957 and later. The specimens were collected by curators of the Natural History Museum or external collaborators.

\section{AMPHIBIANS AND REPTILES}

In the database of Amphibia there are 140 specimens in total, classified in 6 families: RANIDAE $72(51.43 \%)$, with 2 genera and 6 species, SALAMANDRIDAE $22(15.71 \%)$, with 3 genera and 3 species, BOMBINATORIDAE 21 (15\%), with single genus and 2 species, BUFONIDAE 19 (13.57 $\%$ ), with 2 genera and 2 species, Pelobatidae 5 (3.57\%), with single genus and 2 species and HYLIDAE single $(0.71 \%)$, with single genus and single species.

The database contains 13 species of amphibians which are strictly protected in Serbia: Rana dalmatina (23 specimens), Bombina variegata (22 specimens), Salamandra salamandra (15 specimens), Bufo bufo (10 specimens), Bombina bombina (9 specimens), Bufotes viridis (9 specimen), Rana temporaria (7 specimens), Lissotriton vulgaris (5 specimens), Pelobates fuscus (3 specimens), Rana graeca (3 specimens), Ichthyosaura alpestris (2 specimens), Pelobates syriacus (2 specimens) and Hyla arborea (single specimen).

In the database of Reptilia there are 69 specimens in total, classified in 5 families: LACERTIDAE $35(50.72 \%)$, with 3 genera and 4 species, COLUBRIDAE $23(33.33 \%)$, with 4 genera and 5 species, ANGUIDAE 6 $(8.70 \%)$, with single genus and single species, VIPERIDAE 4 (5.80\%), with single genus and 2 species and EMYDIDADE single (1.45\%), with single genus and single species.

The database contains 8 species of reptiles which are strictly protected in Serbia: Natrix tessellata (7 specimens), Dolichopis caspius (5 specimens), Natrix natrix (5 specimens), Zamenis longissimus (5 specimens), Coronella austriaca (2 specimens), Emys orbicularis (single specimen), Zootoca vivipara (single specimen) and Vipera berus (single specimen). 


\section{Acknowledgements}

This research was partly financed by the Ministry of Environmental Protection of the Republic of Serbia and the Institute of Nature Conservation of Serbia - Grants JNOP 01/2018, JNOP 02/2018 and JNOP 03/2018. We gratefully acknowledge Dmitar Lakušić and Gordana Tomović for providing information on these projects.

\section{REFERENCES}

Arnold, E. N., Ovenden, D. (1992): A Field Guide to the Reptiles and Amphibians of Britain and Europe. - Collins, London.

Breitenbach J, Kränzlin F. (1984): Fungi of Switzerland 1 Ascomycetes. - Verlag Mycologia, Luzern.

Ceruti A, Fontana A, Nosenzo C. (2003) Le specie europee del genere Tuber - una revisione storica. - Museo Regionale de Scienze Naturali, Torino.

Euro+Med (2006+): [http://www.emplantbase.org/home.html] (accessed 02.11. 2019)

Horak E. (2005): Röhrlinge und Blätterpilze in Europa. - Elsevier, München.

IF (2019): Index Fungorum. [www.indexfungorum.org] (retrieved October $03^{\text {rd }}$ 2019)

ITIS (2019): Integrated Taxonomic Information System [https://www.itis.gov/] (accessed 22.11.2019)

Montecchi A, Sarasini M. (2000): Fungi ipogei d'Europa. - A.M.B. Fondazione Centro Studi Micologici, Vicenza, Italy.

Moreno-Arroyo B, Gómez J, Pulido E. (2005): Tesoros de nuestro montes. Trufas de Andalucía. - Consejería de Medio Ambiente, Junta de Andalucía, Córdoba.

Niketić, M., Tomović, G. (2018): An annotated checklist of vascular flora of Serbia 1. - Serbian Academy of Sciences and Arts, Belgrade. [in Serbian with English abstract]

Pegler D. N., Spooner B. M., Young T. W. K. (1993): British Truffles: A Revision of British Hypogeous Fungi. - Kew Publishing, London.

POWO (2019): Plants of the World Online. - Royal Botanical Garden Kew. [http://powo.science. kew.org] (retrieved October $11^{\text {th }} 2019$ )

Sarić, M. (ed.) (1992): Flora Srbije ed 2, 1. - Serbian Academy of Sciences and Arts, Belgrade. [in Serbian]

Stevanović, V. (ed.) (2012): Flora Srbije ed 2, 2. - Serbian Academy of Sciences and Arts, Belgrade. [in Serbian]

Tutin, T. G., Burges, N. A., Chater, A. O., Edmondson, J. R., Heywood, V. H., Moore, D. M., Valentine, D. H., Walters, S. M., Webb, D. A. (eds) (1993): Flora Europaea ed. 2, 1. - University Press, Cambridge.

Tutin, T. G., Heywood, V. H., Burges, N. A., Moore, D. M., Valentine, D. H., Walters, S. M., Webb, D. A. (eds) (1968-1980): Flora Europaea 2-5. University Press, Cambridge. 
84 Jovanović, M. ET AL.: Plants, FUnGI, AMPHIBIANS AND REPTILES FROM SERBIA

Tutin, T. G., Heywood, V. H., Burges, N. A., Valentine, D. H., Walters, S. M., Webb, D. A. (eds) (1964): Flora Europaea 1. - University Press, Cambridge.

Zambonelli A, Iotti M, Murat C. (eds) (2016): True Truffle (Tuber spp.) in the World: Soil Ecology, Systematics and Biochemistry. - Springer, Switzerland.

\title{
SUPPORTING INFORMATION
}

Online Appendices:

Appendix 1. - Vascular plants.

Appendix 2. - Fungi.

Appendix 3.- Amphibians and reptiles.

РАСПРОСТРАЫЕЊЕ ОДАБРАНИХ ВАСКУЛАРНИХ БИЉАКА, ГЉИВА, ВОДОЗЕМАЦА И ГМИЗАВАЦА У СРБИЈИ - ПОДАЦИ ИЗ БИОЛОШКИХ ЗБИРКИ ПРИРОДЊАЧКОГ МУЗЕЈА У БЕОГРАДУ КОЈИ СУ СПРОВЕДЕНИ У ОКВИРУ АКТУЕЛНИХ КОНЗЕРВАЦИОНИХ НАЦИОНАЛНИХ ПРОЈЕКАТА

\author{
Р Е 3 И М Е
}

МИРОСЛАВ ЈОВАНОВИЋ, УРОШ БУЗУРОВИЋ, БОРИС ИВАНЧЕВИЋ, АНА ПАУНОВИЋ, МАРЈАН НИКЕТИЋ

Овај каталог је резултат рада аутора Природњачког музеја у Београду који су учествовали у реализацији актуелних пројеката заштите који финансира Завод за заштиту природе Србије. У листама васкуларне флоре, гљива, водоземаца и гмизаваца сумарно је представљено 990 налаза одабраних васкуларних биљака, гљива, водоземаца и гмизаваца из биолошких збирки Природњачког музеја у Београду који се односе на њихову дистрибуцију у Србији. Они укључују 34 представника Polipodiopsida, 32 Pinopsida, 560 Magnoliopsida, 127 Pezizomicetes, 28 Agaricomicetes, 16 Amphibia и 13 Reptilia. База података садржи 86 врста васкуларних биљака, 24 врсте гљива, 13 врста водоземаца и 8 врста гмизаваца, које су заштићене и строго заштићене у Србији. 